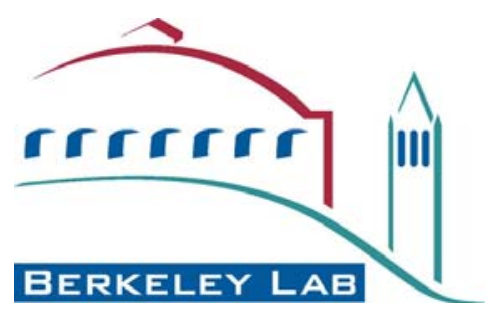

\title{
Modeling Single Well Injection-Withdrawal (SWIW) Tests for Characterization of Complex Fracture-Matrix Systems
}

Collaboration on solute transport modeling in fractured porous rock

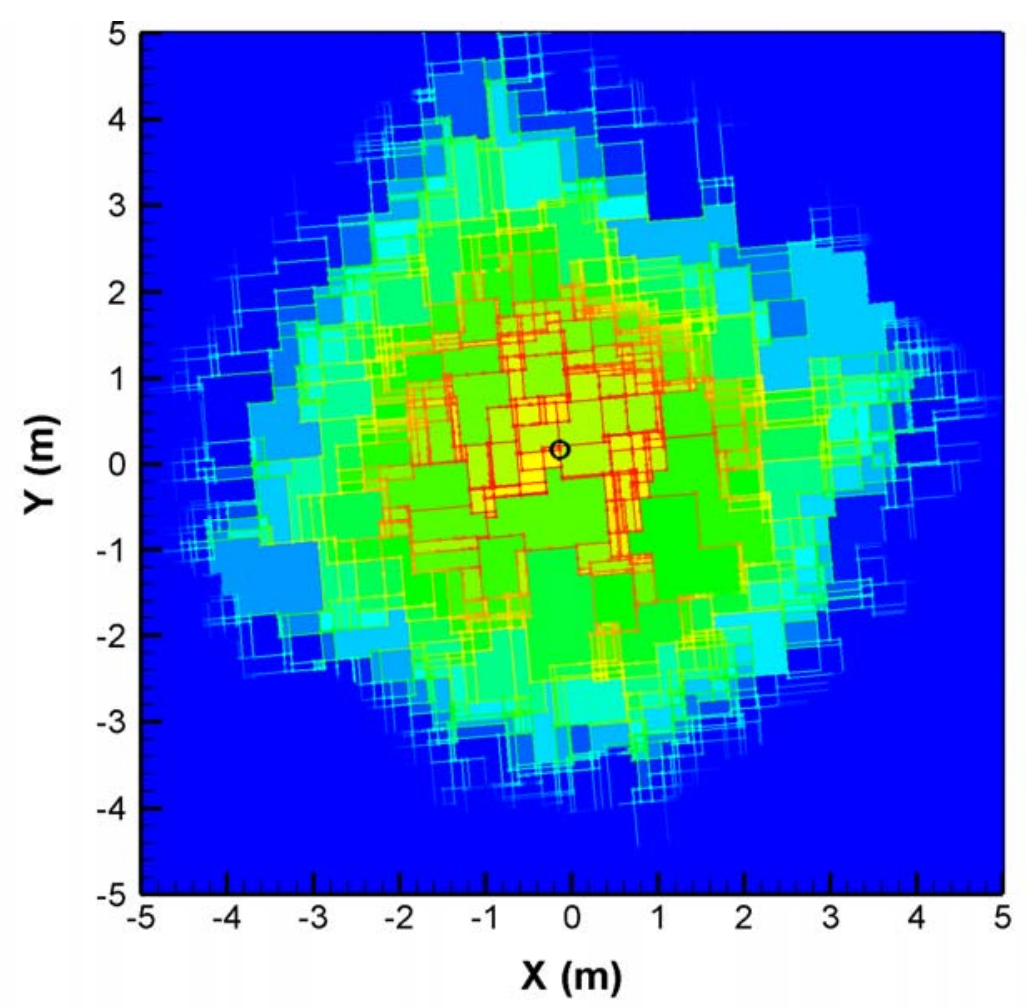

François P. Cotte, Christine Doughty, and Jens Birkholzer

Lawrence Berkeley National Laboratory

Earth Sciences Division

1 Cyclotron Rd

Berkeley, CA 94720

USA

November 2010 


\section{Abstract}

The ability to reliably predict flow and transport in fractured porous rock is an essential condition for performance evaluation of geologic (underground) nuclear waste repositories. In this report, a suite of programs (TRIPOLY code) for calculating and analyzing flow and transport in two-dimensional fracture-matrix systems is used to model single-well injection-withdrawal (SWIW) tracer tests. The SWIW test, a tracer test using one well, is proposed as a useful means of collecting data for site characterization, as well as estimating parameters relevant to tracer diffusion and sorption. After some specific code adaptations, we numerically generated a complex fracture-matrix system for computation of steady-state flow and tracer advection and dispersion in the fracture network, along with solute exchange processes between the fractures and the porous matrix. We then conducted simulations for a hypothetical but workable SWIW test design and completed parameter sensitivity studies on three physical parameters of the rock matrix - namely porosity, diffusion coefficient, and retardation coefficient - in order to investigate their impact on the fracture-matrix solute exchange process. Hydraulic fracturing, or hydrofracking, is also modeled in this study, in two different ways: (1) by increasing the hydraulic aperture for flow in existing fractures and (2) by adding a new set of fractures to the field. The results of all these different tests are analyzed by studying the population of matrix blocks, the tracer spatial distribution, and the breakthrough curves (BTCs) obtained, while performing mass-balance checks and being careful to avoid some numerical mistakes that could occur.

This study clearly demonstrates the importance of matrix effects in the solute transport process, with the sensitivity studies illustrating the increased importance of the matrix in providing a retardation mechanism for radionuclides as matrix porosity, diffusion coefficient, or retardation coefficient increase. Interestingly, model results before and after hydrofracking are insensitive to adding more fractures, while slightly more sensitive to aperture increase, making SWIW tests a possible means of discriminating between these two potential hydrofracking effects. Finally, we investigate the possibility of inferring relevant information regarding the fracture-matrix system physical parameters from the BTCs obtained during SWIW testing.

Keywords: SWIW test, TRIPOLY code, solute transport modeling, complex fracturematrix system, hydrofracking. 


\section{Table of contents}

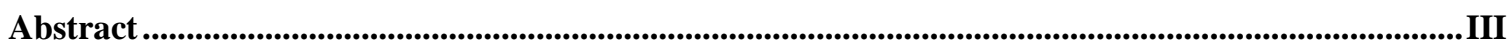

Table of contents............................................................................................................................ IV

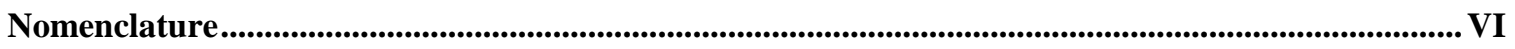

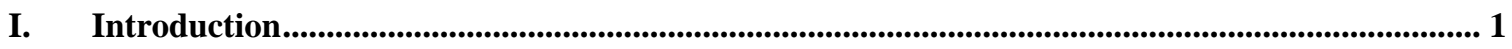

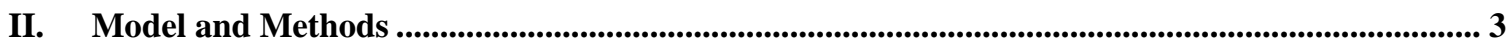

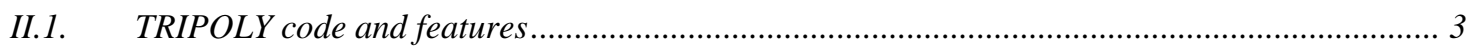

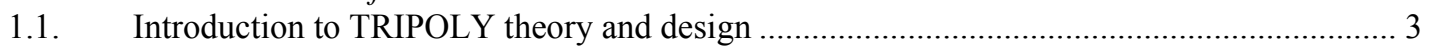

1.2. Advection-dispersion equation and Lagrangian-Eulerian scheme................................... 4

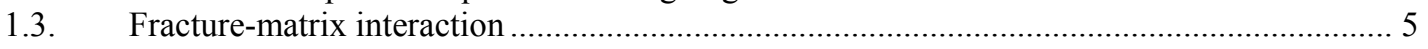

1.3.1 Conceptual model for matrix blocks in a two-dimensional system ............................. 5

1.3.2 Coupling the fracture network with the matrix blocks.......................................... 6

1.3.3 A short remark on the solution scheme and the proximity function concept ................. 7

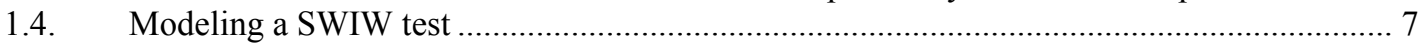

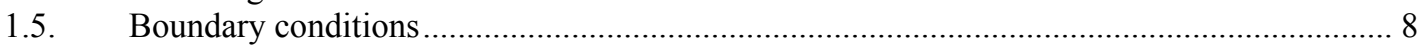

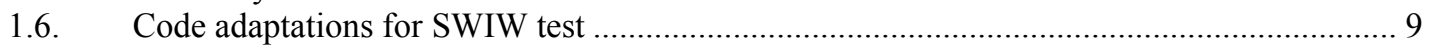

II.2. Sequence of programs and parameter choices ............................................................. 10

2.1. Fracture generation (FMGN) and line network optimizer (RENUMN) ........................ 10

2.2. Matrix blocks geometry calculation (POLY) .............................................................. 11

2.3. Flow and solute transport processes simulation (TRIPOLY) ...................................... 12

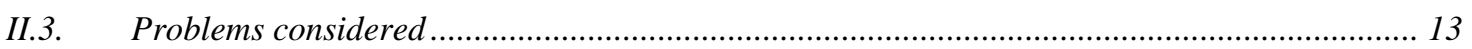

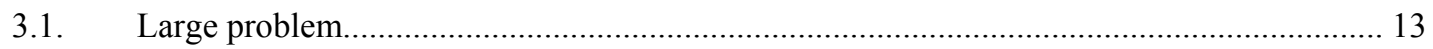

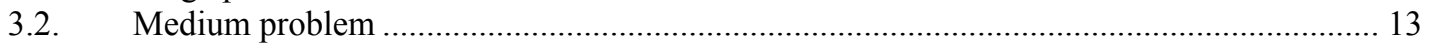

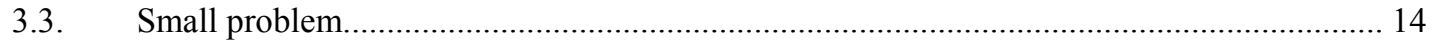

3.4. Parameter sensitivity study and hydrofracking ................................................... 14

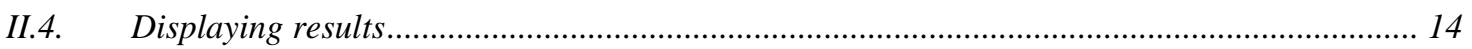

4.1. Population of matrix blocks .............................................................................. 14

4.2. What you see in the field: breakthrough curve and head change..................................... 15

4.3. What we know from the model: spatial distribution and mass balance ............................. 15

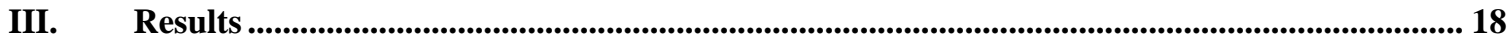

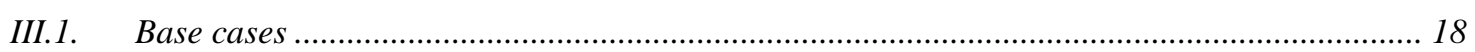

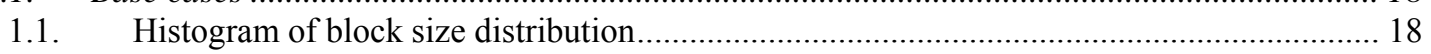

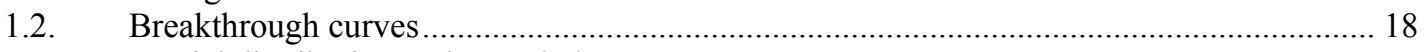

1.3. Spatial distribution and mass balance ..................................................................... 19

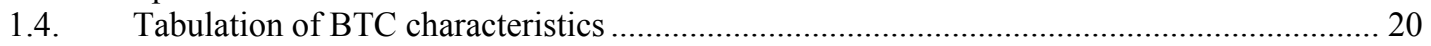

III.2. First parameter sensitivity study: porosity .............................................................. 20

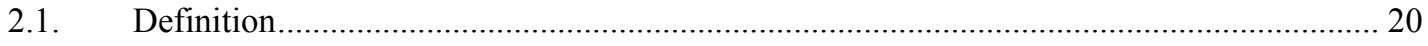

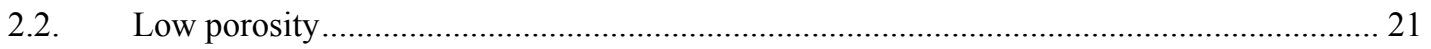

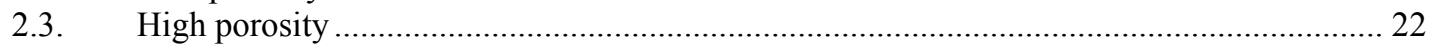

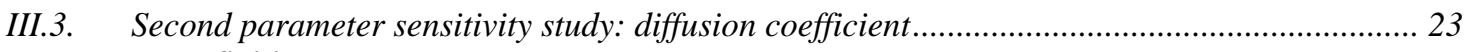

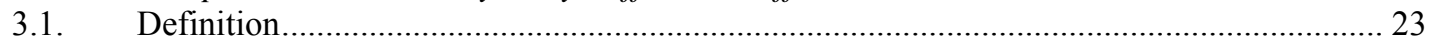

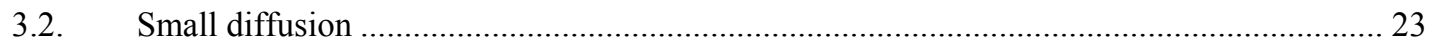

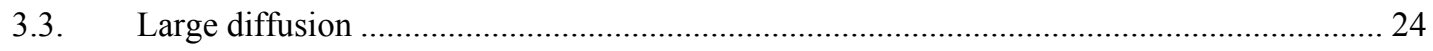


III.4. Third parameter sensitivity study: retardation coefficient ....................................................... 25

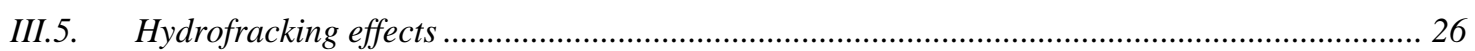

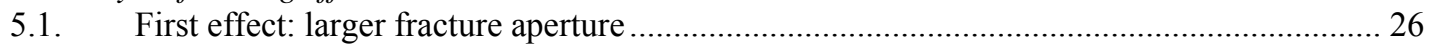

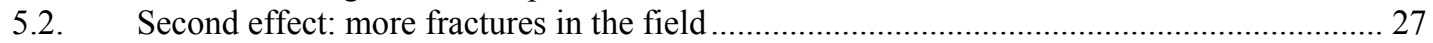

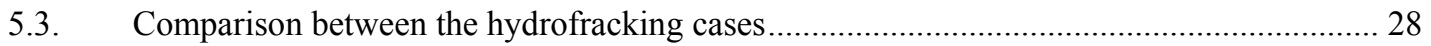

III.6. Comparison between sensitivity cases..................................................................................... 29

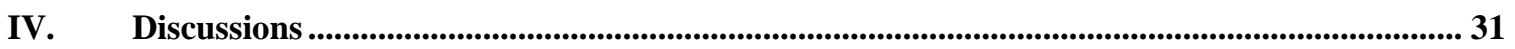

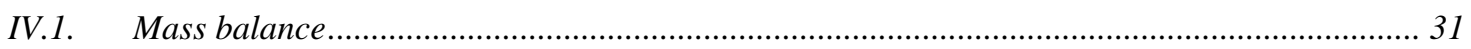

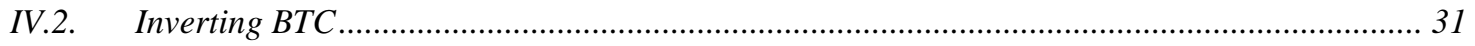

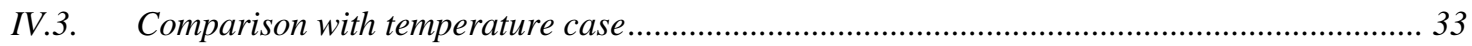

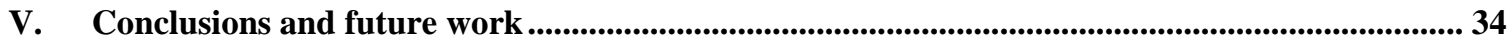

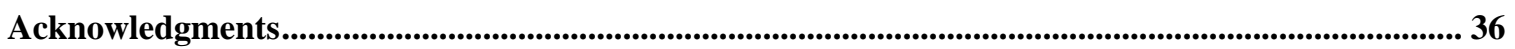

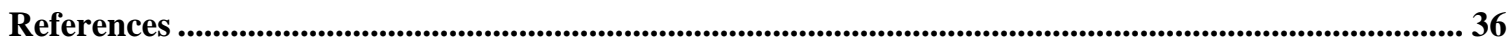

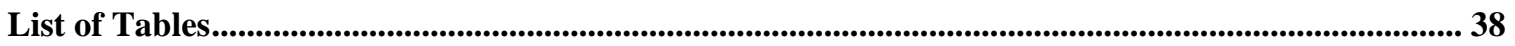

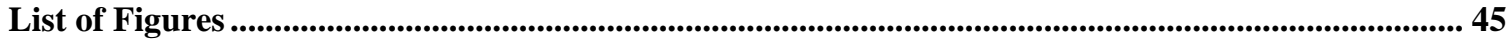




\section{Nomenclature}

\begin{tabular}{|c|c|c|}
\hline Letter & Unit & Significance \\
\hline A & $m^{2}$ & Cross-sectional surface area \\
\hline $\mathrm{A}_{0}$ & $m^{2}$ & Surface area of the matrix block \\
\hline $\mathrm{A}(\mathrm{s})$ & $m^{2}$ & Interface area for transport in the matrix blocks \\
\hline $2 b$ & $m$ & Fracture aperture \\
\hline $\mathrm{C}$ & $g \cdot L^{-1}$ & Relative solute concentration \\
\hline $\mathrm{d}$ & $m$ & Dispersion coefficient \\
\hline $\mathrm{D}_{\mathrm{e}}$ & $m^{2} \cdot s^{-1}$ & Effective diffusion coefficient (in matrix blocks) \\
\hline $\mathrm{D}_{\mathrm{fw}}$ & $m^{2} \cdot s^{-1}$ & Molecular diffusion coefficient in the water (in fractures) \\
\hline g & $m \cdot s^{-2}$ & Gravitational constant \\
\hline $\mathrm{H}$ & $m$ & Hydraulic head \\
\hline K & $m^{2}$ & Permeability \\
\hline $\mathrm{K}_{\mathrm{D}}$ & $\mathrm{m}^{3} \mathrm{~kg}^{-1}$ & Sorption coefficient or distribution coefficient for the solute with the rock \\
\hline $\mathrm{L}$ & $m$ & Fracture length \\
\hline M & $g$ & Mass of introduced tracer \\
\hline $\operatorname{Prox}(\mathrm{s})$ & & Proximity function \\
\hline q & $m^{3} \cdot s^{-1}$ & Flow rate \\
\hline $\mathrm{r}$ & & Recovery factor \\
\hline $\mathrm{R}$ & & Retardation coefficient \\
\hline $\mathrm{S}$ & $m$ & Maximum available distance from fracture to a considered block center \\
\hline $\mathrm{s}$ & $m$ & Local coordinate perpendicular to adjacent fractures \\
\hline $\mathrm{t}$ & $S$ & Time \\
\hline $\mathrm{u}$ & $m \cdot s^{-1}$ & Pore velocity \\
\hline $\mathrm{V}$ & $m \cdot s^{-1}$ & Darcy velocity through the medium, where $v=q / A$ \\
\hline $\mathrm{V}_{\mathrm{T}}$ & $m^{3}$ & Total block volume \\
\hline $\mathrm{V}_{\mathrm{V}}$ & $m^{3}$ & Volume of void space \\
\hline $\mathrm{V}(\mathrm{s})$ & $m^{3}$ & Total fraction of matrix volume within a distance s from the adjacent fractures \\
\hline $\mathrm{W}^{\mathrm{D}}$ & & Diffusive loss into the matrix on fracture wall \\
\hline $\mathrm{x}$ & $m$ & Position axis $\mathrm{x}$ \\
\hline$x^{\prime}$ & $m$ & Coordinate defined parallel to the fracture axis \\
\hline $\mathrm{y}$ & $m$ & Position axis y \\
\hline
\end{tabular}




\begin{tabular}{|c|c|c|}
\hline Symbol & Unit & Significance \\
\hline$\alpha$ & $m$ & Dispersion coefficient (or longitudinal dispersivity in fracture elements) \\
\hline$\Delta \mathrm{P}$ & $\mathrm{Pa}$ & Pressure difference \\
\hline$\nabla H$ & & Hydraulic head gradient \\
\hline$\nabla P$ & $\mathrm{Pam}^{-1}$ & Pressure gradient \\
\hline$\Phi$ & & Porosity \\
\hline$\mu$ & $\mathrm{Pa}$ is & Dynamic viscosity of the fluid \\
\hline$\rho$ & $\mathrm{kg} \cdot \mathrm{m}^{-3}$ & Density of fluid \\
\hline$\rho_{P}$ & $\mathrm{~kg} \cdot \mathrm{m}^{-3}$ & Rock density \\
\hline$\tau$ & & Tortuosity \\
\hline
\end{tabular}

$\begin{array}{ll}\text { Subscript - Superscript } & \text { Significance } \\ \text { C } & \text { Chase fluid } \\ \text { F } & \text { Fracture } \\ \text { i } & \text { Bin time } \\ \text { I } & \text { Injection } \\ \text { M } & \text { Matrix } \\ \text { W } & \text { Withdrawal }\end{array}$

$\begin{array}{ll}\text { Abbreviation } & \text { Significance } \\ \text { BTC } & \text { Breakthrough Curve } \\ \text { MB } & \text { Mass Balance } \\ \text { SWIW } & \text { Single-Well Injection-Withdrawal }\end{array}$




\section{Introduction}

The capability of reliably predicting flow and transport in fractured porous rock is a vital requirement for performance assessment of many geologic repositories for nuclear or toxic waste. Additionally, such capability leads to a better understanding of rocks under extreme conditions, such as in oil, gas, or geothermal energy exploitation. The transport behavior in such systems is characterized by fast transport in connected fractures coupled with slow diffusion into the porous rock matrix, the latter an important retardation mechanism for radionuclides. The size and shape distribution of matrix blocks, together with porosity, diffusion, and sorption properties of the rock, control this retardation mechanism.

A wide range of techniques are available to carry out hydrologic and tracer tests in support of characterizing flow and transport in fractured porous rock. Hydrologic tests can involve one or more wells, whereas tracer tests typically involve two or more wells. It has been demonstrated, thanks to several previous studies (Haggerty et al., 2001 [7]; Schroth et al., 2001 [15]; Nordqvist and Gustafsson, 2002 [11]; Nordqvist and Gustafsson, 2004 [12]; Neretnieks, 2007 [10]; Gouze et al., 2008 [6]) that the single-well injection-withdrawal (SWIW) test, a tracer test using only one well, is useful in site characterization of fractured rock as well as in providing information on tracer transport diffusive properties.

The usual conceptual model of flow and transport through fractured rock involves advection and dispersion through the fracture network, coupled with diffusion and sorption into the surrounding rock matrix. In a SWIW tracer test, one well injects fluid and tracer at a constant rate for a period of time, followed by injection of fluid (called chase fluid) without tracer for a certain amount of time. Then the pump is reversed and the well withdraws fluid at the same rate until most (or all) the tracer is retrieved. Unlike a typical two-well tracer test, which is typically very sensitive to advective heterogeneity, in SWIW tests, involving reversing flow fields by injection and then withdrawal at the same flow rate, these advective heterogeneities tend to cancel out. Thus, SWIW-test results are ideally independent of advective heterogeneity, flow channeling, and flow dimension. As a result, the breakthrough curve (BTC) is not sensitive to this "advective dispersivity" but is dominated by the presence of matrix diffusion (as well as other processes affecting solute exchange between fracture and matrix) (Doughty and Tsang, 2009 [5]). Hence, matrix diffusion has to be taken into account in the advectiondispersion equation used to model flow and transport through the fracture network.

One key difference between a two-well test and a SWIW test is illustrated in Figure 1. In a two-well test, particles always "see" new rock matrix, whereas in a SWIW test, they return past the same rock matrix during the withdrawal period that they already passed during the injection phase. If this rock matrix is composed of finite blocks, the latter may become "saturated" with tracer (i.e., $\mathrm{C}^{\mathrm{M}}=\mathrm{C}^{\mathrm{F}}$ ) in a SWIW test, therefore accepting no more diffusion and sorption. Another key difference resides in the fact that travel distance 
is essentially fixed for two-well tests, set by well separation, so that the peak arrival time may change, and thus time for diffusion may vary as well, whereas travel time is essentially fixed for SWIW tests, set by the schedule of the test, so that the tracer plume size (or spatial distribution) may change. Compared to a typical two-well test, a SWIW test is expected to produce a higher tracer recovery ratio, be more feasible in the field and provide information on the flow wetted surface of a fracture network - that is, the contact surface area between flowing water in fractures and the rock (Tsang and Doughty, 2009 [17]).

The purpose of this study is to evaluate the SWIW method for investigating complex fracture-matrix systems. Using the TRIPOLY simulator developed at LBNL by Birkholzer and Karasaki, 1996 [3] and a suite of pre- and postprocessing codes, such systems are numerically generated based on geostatistical data, and then flow and transport simulations are conducted for a hypothetical SWIW test. The numerical predictions for breakthrough curves are then interpreted with the goals of (1) verifying the basic physical processes occurring in the fracture network and the matrix system, and (2) developing methods for inversely extracting, from the BTC data, information on the impact of hydrofracking and the relevant diffusion and sorption parameters. These are hard to measure otherwise, in particular with respect to the size and shape distribution of matrix blocks.

Most previous studies with TRIPOLY considered the fracture network only, such as Doughty and Karasaki, 2002 [4]. Previous SWIW-test analyses (Doughty and Tsang, 2009 [5]; Tsang and Doughty, 2009 [17]; Pruess and Doughty, 2010 [14]) considered only a single fracture and either a semi-infinite matrix only or a semi-infinite matrix plus finite matrix blocks all with the same size. In the present study, a two-dimensional network of fractures creates a population of matrix blocks with a wide range of sizes, with the goal of verifying basic flow and transport processes that occur in the fracture network and the matrix system.

We present the model and the methods used in Section II, then Section III describes the results obtained, followed by additional discussion in Section IV. Section V provides conclusions and describes future work that could be done. 


\section{Model and Methods}

The following sections represent the first step of the project. They will describe the original TRIPOLY code (Birkholzer and Karasaki, 1996 [3]), the modifications made to the suite of programs used to run TRIPOLY for the present problem, the parameter choices made, and how results will be displayed.

\section{II.1. TRIPOLY code and features}

The suite of programs used to run TRIPOLY aims to simulate flow and solute transport in two-dimensional fracture networks surrounded by porous matrix blocks. Three preprocessing codes need to be utilized before running TRIPOLY simulations. These codes generate fracture networks (FMGN), optimize the network numbering and eliminate dead-ends if desired (RENUMN), and calculate matrix block shapes and properties (POLY), and are described in Section II.2. A flow chart for use of the programs is shown in Figure 2.

\subsection{Introduction to TRIPOLY theory and design}

Here we briefly describe the general background of TRIPOLY and the theory of treating the fluid and solute exchange between fractures and rocks. A complete description of the theory and design of TRIPOLY is provided by Birkholzer and Karasaki, 1996 [3]. The main feature of this code is that it enables us to study plume spreading processes in fractured porous rock.

Fracture network models are useful tools for better understanding the hydrogeology of fractured rock. Once the geometry of a particular region has been fixed, flow and solute transport processes through the network and surrounding rock can then be studied using appropriate numerical procedures. A tool for studying such processes already exists: TRINET code (Karasaki, 1986 [8]; Segan and Karasaki, 1993 [16]). It solves the advection-dispersion equation with a mixed Lagrangian-Eulerian scheme, combined with adaptive gridding techniques. But it is limited in that it does not account for solute exchange between the fractures and the porous matrix.

That is the reason why we use TRIPOLY: it combines the fracture network simulator TRINET with advanced numerical methods to account for fracture-matrix interaction. The basic approach consists of treating fractures and matrix blocks as two different systems, and the interaction between the two systems is modeled by introducing some shared terms in both systems. It is assumed that transport in the matrix can be approximated as a one-dimensional diffusion process, perpendicular to the adjacent fracture surfaces. Under that assumption, the geometrical shape of the individual matrix blocks can be described by proximity functions, which determine the fraction of matrix 
volume within a certain distance from the adjacent fractures. Thus, a direct solution scheme is employed to solve the coupled fracture and matrix equations, which will be described in Section 1.3.

\subsection{Advection-dispersion equation and Lagrangian-Eulerian scheme}

The advection-dispersion equation for advective-dispersive transport in discrete fractures is based on the conservation of mass and is applicable when Darcy's law is valid. The latter provides an accurate description of groundwater flow in almost all hydrogeological environments. Darcy's law in a one-dimensional (1D) system can be defined as follows:

$$
q=-\frac{K \cdot A}{\mu} \nabla P
$$

where $q$ is the flow rate of the considered fluid in $\mathrm{m}^{3} \cdot \mathrm{s}^{-1}, K$ is permeability in $\mathrm{m}^{2}, A$ is the cross-sectional area in $\mathrm{m}^{2}, \mu$ is the dynamic viscosity of the fluid in Pa.s (or $\mathrm{kg} \mathrm{m}^{-1} \cdot \mathrm{s}^{-1}$ ) and $\nabla P$ is the pressure gradient.

Another form of Darcy's law in 1D can be expressed as follows:

$$
v=\frac{q}{A}=-\frac{K \rho g}{\mu} \nabla H
$$

where $v$ is Darcy velocity in $\mathrm{m}_{\mathrm{s}} \mathrm{s}^{-1}, \rho$ is density of fluid in $\mathrm{kg} . \mathrm{m}^{-3}, g$ is gravitational constant in $\mathrm{m} . \mathrm{s}^{-2}$, and $\nabla H$ is the hydraulic head gradient. Note that $P$ and $H$ can also be related by the following formula: $P=\rho g H$. In our considered problem, rock density $\rho$ is constant, as well as gravity $g$, so we can assume that $\nabla P \sim \nabla H$.

The pore velocity $u$ can also be introduced by the following formula:

$$
u=\frac{q}{A \Phi^{F}}=\frac{v}{\Phi^{F}}
$$

where $u$ is pore velocity in $m \cdot s^{-1}$ and $\Phi^{F}$ is porosity in the fracture.

The advection-dispersion equation in a one-dimensional system (along the $\mathrm{x}$ axis, for instance) can be written as follows:

$$
\frac{\partial C}{\partial t}=\underbrace{\alpha_{x} \frac{\partial^{2} C}{\partial^{2} t}}_{\text {dispersive transport }}-\underbrace{u_{x} \frac{\partial C}{\partial x}}_{\text {advective transport }}
$$

where $C$ is the relative solute concentration, $\alpha$ is the dispersion coefficient, and $u$ is the pore velocity. Note that the two coefficients $\left(\alpha_{x}\right.$ and $\left.u_{x}\right)$ are constant under the assumption of a steady-state flow field. Moreover, the hydrodynamic dispersion term usually also includes molecular diffusion, and advection can also be known as convection. 
Then, the idea in using a mixed Lagrangian-Eulerian scheme is to decompose the advection-dispersion equation into two parts, one controlled only by advection, the other by dispersion. The advected concentration profiles are calculated by Lagrangian approaches (such as particle tracking methods), whereas the dispersed concentration profiles are solved by conventional numerical techniques on Eulerian grids (such as finite difference methods or finite element methods). Furthermore, adaptive gridding schemes are combined with the advection part, introducing forward-moving particles around sharp fronts. However, numerical dispersion may occur when the advected front is projected back to the fixed Eulerian grid. That implies that the accuracy of results is dependent on the number of particles introduced into the model.

TRIPOLY features two major improvements compared to the above-mentioned methods. First, advective tracking in the fracture network is performed for nodal concentrations and not for particles. Therefore, the number of particles introduced is not a concern. Second, numerical dispersion is minimized by creating new Eulerian grid points instead of interpolating the advected profile back to a fixed Eulerian grid.

\subsection{Fracture-matrix interaction}

\subsubsection{Conceptual model for matrix blocks in a two-dimensional system}

In this section, the theory of treating fracture-matrix interaction is presented. The advective-dispersive flow problem for the fracture network is assumed to be already solved as described above. The model is limited to two-dimensional fracture-matrix systems, but it could be easily extended to three-dimensional systems.

As noted above, TRIPOLY assumes that global transport processes take place only in the fracture network: the rock medium does not contribute to those processes. However, concentration differences between the fractures and the matrix lead to a diffusive solute exchange at the fracture-matrix interface, and fractions of the solute may be stored in matrix pores. Since the diffusive transport in the matrix is much slower than the coupled advective-dispersive spreading in the fractures, it can be approximated as a onedimensional process, perpendicular to the adjacent fractures. Under these assumptions, a one-dimensional mass-balance equation describing the diffusive transport in single matrix blocks can be written as follows:

$$
\Phi^{M} R^{M} \frac{A(s)}{A_{0}} \frac{\partial C^{M}}{\partial t}-\Phi^{M} D_{e}^{M} \frac{\partial}{\partial s}\left(\frac{A(s)}{A_{0}} \frac{\partial C^{M}}{\partial s}\right)=0
$$

where $C^{M}$ is the relative concentration in the matrix, $\Phi^{M}$ is matrix porosity, $R^{M}$ is the retardation coefficient, $D_{e}^{M}$ is the effective diffusion coefficient in the matrix, $A(s)$ is the interface area for transport in the matrix blocks, $A_{0}$ is the surface area of the matrix blocks and $s$ is a local coordinate perpendicular to the adjacent fractures. Further details regarding the model used for diffusive transport in single matrix blocks are provided in Figure 3. 
Two boundary conditions are also added to solve the local fracture-matrix diffusion problem:

$$
\begin{gathered}
C^{M}(s=0)=C^{F} \\
\frac{\partial C^{M}}{\partial s}(s=S)=0
\end{gathered}
$$

where the first boundary condition describes the fact that concentrations in the fracture and in the matrix blocks are equal at the fracture-matrix interface, and Equation (7) is a zero-flux boundary condition in the middle of the matrix blocks. These boundary conditions imply that the diffusion equations for individual matrix blocks are independent from each other.

\subsubsection{Coupling the fracture network with the matrix blocks}

After solving Equation (5), the diffusive solute exchange term $W$ per unit fracture wall area is obtained by applying Fick's law at the interface between fractures and porous blocks, using the following formula:

$$
W^{D}=\left.\Phi^{M} D_{e}^{M} \frac{\partial C^{M}}{\partial s}\right|_{s=0}
$$

where $W^{D}$ can also be defined as the coupling term between the dispersion-diffusion equation of the fracture network and the diffusion equation of the individual matrix blocks.

Hence, with the fracture network and the matrix blocks now coupled by the solute exchange term $W$ defined by Equation (8), the dispersion equation for a single fracture can be written as follows:

$$
\frac{\partial C^{F}}{\partial t}-\alpha^{F} \frac{\partial^{2} C^{F}}{\partial x^{\prime 2}}+\frac{W^{D 1}+W^{D 2}}{(2 b)}=0
$$

where $C^{F}$ is the relative concentration in the fracture, $\alpha^{F}$ is the dispersion coefficient in the fracture, $(2 b)$ is the fracture aperture, $x^{\prime}$ is a coordinate defined parallel to the fracture axis, and $W^{D 1}$ and $W^{D 2}$ can be interpreted as the diffusive losses into the matrix on fracture wall one and two, in that order.

The concept of coupling the fracture network and the matrix blocks is illustrated by Figure 4. Each matrix block is defined by its material properties (such as porosity $\Phi^{\mathrm{M}}$ and diffusion coefficient $\mathrm{D}_{\mathrm{e}}$ ), by geometrical parameters (interface function and block size) and by its surface polygon, which is described by the node numbers of the surrounding fractures. In the meantime, each node of the fracture network is connected to a certain number of blocks, or polygons, each related to a one-dimensional concentration 
distribution in the matrix. For each connection, the solute exchange is calculated by Equation (8) and the resulting exchange rate is then introduced into Equation (9).

\subsubsection{A short remark on the solution scheme and the proximity function concept}

Each individual matrix block in the modeled domain is associated with a certain number of one-dimensional independent diffusion equations describing the local transport in the matrix (i.e., one equation for each fracture bounding the matrix block). However, each of these equations is coupled to the advection-dispersion equation of the fracture network by the use of the solute exchange $W^{D}$ defined by Equation (8). The numerical solution procedure chosen for the coupled equation system of fractures and matrix is that the two media are discretized and solved separately. For further details on the solution procedure, please refer to Birkholzer and Karasaki, 1996 [3].

According to the one-dimensional diffusion Equation (5), interface function $A(s)$ must be provided, which defines the interface area for diffusive transport at a distance $s$ from the block surface. In TRIPOLY, interface functions for each block are defined following the concept of proximity functions, which has been described in Pruess and Karasaki, 1982 [13]. The proximity function Prox(s) can be expressed as

$$
\operatorname{Prox}(\mathrm{s})=\frac{V(s)}{V_{T}}
$$

where $V(s)$ stands for the total fraction of matrix volume within a distance $s$ from the adjacent fractures, and $V_{T}$ is the total block volume.

Thus, the interface area for diffusive flux in the matrix is simply defined as follows:

$$
A(s)=\frac{d V(s)}{d V}=V_{T} \frac{d \operatorname{Prox}(\mathrm{s})}{d s}
$$

Finally, in relation to Equation (5), the interface function $A(s)$ is divided by the actual surface area $A_{0}$ of each matrix block in order to normalize the values.

\subsection{Modeling a SWIW test}

As briefly described in the introduction, a Single Well Injection-Withdrawal test (SWIW test) is typically divided into four main parts: the injection period, the chase fluid period, the rest time, and the withdrawal period. Concerning the injection part, the well injects both fluid and tracer at a constant rate for a certain period of time. Assuming a relative tracer concentration of 1.0, we must specify injection time and flow rate, i.e., how much tracer will be injected into the system. Regarding the chase fluid period, the duration of this part is hard to estimate: it has to be "both sufficiently long and short." In other words, a too short chase fluid period will not impact the previously injected tracer enough, while a too long chase fluid period would spread too much tracer outside our domain of study. During the chase fluid period, only untraced water is injected, using the same rate as during the injection period. Regarding the rest time, this period can be used to ensure that 
the tracer has plenty of opportunity to penetrate the matrix blocks. In our case, we decided to delete this step - this quiescent time is not considered essential for a SWIW test. The last part of a SWIW test is the withdrawal period. Typically, it consists of reversing the pump (the well withdraws fluid at the same rate that was previously chosen for injection) until most of the tracer is recovered. The only issue is to settle on the duration, that is, the fraction of injected tracer we would like to retrieve, while knowing it may take a long time to achieve this.

Table 1 summarizes the parameters that have to be selected in order to run a SWIW test.

\subsection{Boundary conditions}

The choice of boundary conditions is fundamental in this study: while several possibilities exist, various tests are made to choose both the most efficient and most physically adequate conditions. Boundary conditions need to be provided only for a few nodes: the nodes located at the edge of our domain and the well node for injection and extraction of fluids.

Regarding the flow equation, either a Dirichlet condition (i.e., fixed head) or a Neumann condition (i.e. fixed water flux) can be set. Regarding nodes at the edge of the domain, we are injecting fluid and tracer into the domain and consequently allowing some flow to leave our area of study. Therefore, a Dirichlet boundary condition is imposed at any outside boundary node, with a prescribed constant hydraulic head equal to zero. Because we are only interested in head differences, we choose $H=0$ for convenience. Setting all boundary heads to the same value is equivalent to assuming that there is negligible regional flow naturally in the system. Concerning the well node, since the desired flow rate is known, a Neumann condition is set with a given value for the flux, the determination of which will be described later in this report.

Then, a boundary condition must be imposed for solute transport at these same nodes. Once again, either a Dirichlet condition or a Neumann condition can be chosen. If we focus on outside boundaries, some trials are performed with a Dirichlet condition, i.e., a fixed concentration set to $\mathrm{C}=0$. But in this particular case, we are losing information, such as the loss of tracer during the injection and chase fluid periods. Besides, this specific condition is not very representative of the physical reality in the field (even though it would be convenient for modeling): the probability that some tracer arrives at the boundaries is high, depending on the amount of tracer injected, or depending on some preferential paths that could be taken. As a consequence, we finally decided to treat these particular nodes as internal nodes, where no specific boundary condition is assigned. However, the code internally treats these boundary nodes as Cauchy conditions, which means that fluids leaving the model domain will carry with them any solute they might contain.

The same problem arises with the well node. Injecting a tracer at a given relative concentration of 1.0 (Dirichlet condition) or at a given flow rate over a certain amount of 
time (Neumann condition) may seem equivalent in terms of "final result," but these two options are physically different. We determine that a Neumann condition is more appropriate, because it accounts for the gradual increase in tracer concentration as the elements surrounding the injection node fill with tracer, while the Dirichlet condition merely imposes a given concentration that is instantly reached. Hence, a Neumann condition is assigned to the well node during the injection time with a specific value $q_{I}$ depending on the considered problem.

Finally, once the boundary conditions are all set for the injection period, some of them must be adjusted during the process of the SWIW test. Regarding outside boundaries, there is no change needed during the entire process. Regarding the well node, once the injection period is over, no more tracer is injected, but water, i.e., a fluid with no tracer, continues to be injected, representing the chase period. Thus, the boundary condition value for solute transport has to be switched from the initial value of $q_{I}$ to $q_{I}=0$. Note that the node can be either treated in this manner or considered as an internal node for solute transport. Indeed, the code injects water by default into the system, making these two choices indistinguishable. Eventually, once the withdrawal period begins, the tracer is pulled back, so the pump is reversed. This implies that the flow value of the fixed flux must be reversed as well, while keeping the node as an internal node for solute transport.

Table 2 recapitulates all the boundary conditions used in a SWIW test.

\subsection{Code adaptations for SWIW test}

Some changes had to be carried out to adapt TRIPOLY to our case. Indeed, TRIPOLY was created to simulate flow coupled with solute transport moving through the generated fracture-matrix network. Thus, the code was specially designed to introduce tracer entering the fracture network from one side, with appropriate boundary conditions, with this information hard-wired into the code itself. This particular problem has been addressed by removing those specific tracer boundary conditions from the code, thus allowing the user to specify all boundary conditions through input files. Moreover, it was discovered that the code was injecting only tracer by default; it did not allow injection of water together with tracer. This limitation was changed in the revised code. Furthermore, the code needed to be adapted to model the different time periods of a SWIW test within one simulation. This was accomplished by adding a new feature in the code to modify boundary conditions during code execution. Also, a new feature was added to calculate the total tracer mass in the fractures and the matrix for each time step. Lastly, a final code adaptation had to be performed to fix some crashing problems at the end of our simulations. 


\section{II.2. Sequence of programs and parameter choices}

\subsection{Fracture generation (FMGN) and line network optimizer (RENUMN)}

The main purpose of FMGN is to generate a two-dimensional fracture network, with any desired distribution of aperture, length, and orientation for each fracture set that composes our model. FMGN is a new version of FMG (Fracture Mesh Generator), whose theory and design are thoroughly described in Billaux et al., 1988 [1]. The fracture system generated by FMGN may include dead-end fractures.

FMGN enables us to choose the shape of the generation and flow regions, namely a rectangular region or a circular region with a circular hole in the flow region. Even though the second option would seem appropriate for our case, it was finally decided to work with a rectangular region, because (1) it can be easily transposed to the field and (2) the rectangular generation is a standard choice for directional permeability measurements. The well would then be modeled as a specific node located as close as possible to the middle of the generated rectangular area.

Next, we specify parameters for the different fracture sets in order to generate the fracture network. This includes the number of sets, the number of fractures per set, the orientation, length, and aperture for each fracture of each set, and the dimension of the rectangular region. It should also be noted that other parameters exist, but they are set to their default value. For instance, a standard deviation is not taken into account in the statistical distribution of orientation, length, and aperture values, nor is an angle of rotation within the flow region employed.

As a result, we generate two orthogonal sets of 700 fractures each, in a $10 \mathrm{~m} \times 10 \mathrm{~m}$ square flow region. The angle between the fracture axis and the $\mathrm{x}$-direction is $5^{\circ}$ for the first set and $95^{\circ}$ for the second set. It is assumed that the fractures have uniform properties within each set - that is to say, a uniform fracture aperture of $1110^{-4} \mathrm{~m}$, a uniform fracture length of $1.0 \mathrm{~m}$, and uniform angles between the fractures and the $\mathrm{x}$ axis.

Additionally, some outside boundary conditions have to be specified at this point. In our case, all the boundaries have a constant hydraulic head fixed to $H=0$ for convenience. Table 3 provides a recap of all the chosen features used to generate the fracture network geometry. Figure 5 shows the generated geometry using FMGN.

Once FMGN has been executed, RENUMN reads the output generated by FMGN so as to generate and to optimize the finite-element mesh for the fracture network. Specifically, RENUMN merges nodes very close to each other, removes dead-end fractures if desired, renumbers the nodes to minimize the bandwidth of the linear equation system to be solved in TRIPOLY, and creates the POLY and TRIPOLY input files of the fracture network. For further details on the theory and design of RENUMN, please refer to the 
two reports written by Billaux et al., 1988 [1] and 1989 [2]. RENUMN provides some general information on the material parameters of the fracture network, such as dispersion, values for dynamic viscosity, etc. The only significant modification to the default settings concerns the dispersion coefficient $\alpha$, which has to be taken into account and is therefore set to $0.5 \mathrm{~m}$.

\subsection{Matrix blocks geometry calculation (POLY)}

The third program to be executed, POLY, calculates the geometrical properties of the porous matrix blocks surrounding the fracture network given by FMGN. In our particular case, POLY is critical because solute exchange processes between the fracture network and the porous matrix are taken into account; in other words, matrix diffusion is considered.

POLY calculates the matrix block geometry. It starts by extending dead-end fractures until they intersect either another fracture or the flow-region boundary. Then, as porous matrix blocks are described by polygons created by RENUMN, POLY determines the geometrical shape of the matrix blocks by using the proximity function and the maximum orthogonal distance of the block center to the nearest fracture. Figure 6 illustrates the work done by POLY. The theory and use of POLY is fully described in another report (Birkholzer and Karasaki, 1996 [3]).

POLY requires information about the network with dead-ends. These input files are provided by RENUMN. However, it is possible to choose whether or not the output polygons (and proximity functions) provided to the simulation code TRIPOLY should include dead-end fractures. In our case, we assume that the impact of dead-end fractures can be neglected, so the polygons for fracture network do not include dead-ends. This hypothesis turns out to be confirmed by some tests carried out in this context.

Another input file contains the material properties for matrix blocks: the porosity $\Phi^{M}$, the diffusion coefficient $D_{e}$ and the retardation coefficient $R$. A number of control variables for the generation of polygons and proximity functions can be modified at this point as well.

The retardation coefficient accounts for sorption. This retardation factor may be written as follows:

$$
R=1+\frac{1-\Phi^{M}}{\Phi^{M}} \rho_{P} K_{D}
$$

where $\Phi^{M}$ is porosity in matrix blocks, $\rho_{P}$ is rock density in $\mathrm{kg} \cdot \mathrm{m}^{-3}$, and $K_{D}$ is sorption coefficient or distribution coefficient in $\mathrm{m}^{3} \cdot \mathrm{kg}^{-1}$ (see Birkholzer and Karasaki, 1996 [3] and Pruess and Doughty, 2010 [14] for more details on retardation coefficient). It should be noted that $R=1$ implies that a nonsorbing tracer is used $\left(K_{D}=0\right)$, whereas $R \neq 1$ means that sorption is taken into account, so that the tracer can be considered as nonconservative. An example of a nonsorbing tracer that could be used would be uranine, 
whereas rubidium and cesium are considered to be sorbing tracers (Doughty and Tsang, $2009[5])$.

At this point, it should be stressed that realizing the numerical solutions of advectiondispersion processes in a fracture network is a complex task, one in which instabilities and numerical problems can occur. In our particular case, the geometry created by FMGN must be physically and numerically feasible; otherwise POLY is unable to link the fractures together. An example of "bad geometry" is provided in Figure 7, a case in which the fracture network has insufficient connectivity. Furthermore, if the geometry is either too complex or includes too many fractures, the program can either crash or be blocked at some point. This problem can sometimes be solved by decreasing a certain precision or tolerance, but this can lead to a decrease in accuracy.

\subsection{Flow and solute transport processes simulation (TRIPOLY)}

TRIPOLY is the final step in our chain of simulating flow and solute transport processes in fracture-matrix systems. TRIPOLY solves for flow and transport in two-dimensional fracture networks, taking into account solute exchange processes between the fractures and the porous matrix. Full details about the theory and use of the code are described in Birkholzer and Karasaki, 1996 [3].

TRIPOLY is primarily governed by one input file named ctrl.inp, which contains all the general information and the main control variables that are needed to simulate a SWIW test. To begin with, the type of simulation can be controlled by two variables: IDOPO and IMODE. The former determines whether the porous matrix is considered, the latter enables us to choose between a steady-state and a transient flow field. Initial testing indicates that using a transient flow field is a problem for the code. Ultimately, we assume that the transient flow can be neglected for our simulations, because compared to the duration of each stage of the SWIW test, the transient flow that arises at the transition between stages is nearly insignificant. Hence the establishment of steady flow is considered instantaneous for each stage in the SWIW test.

Another key issue to consider carefully is time-stepping. Indeed, as a matter of accuracy and consistency, it has been shown that TRIPOLY is very sensitive to the chosen time step sequence (Birkholzer and Karasaki, 1996 [3]; Najita and Doughty, 1998 [9]). According to the numerous runs carried out in this context, optimal values are selected for the variable $P R R$ (the factor for increasing the time step) and the different output time steps specified for writing the desired output data sets.

After that, some numerical parameters need to be carefully chosen, in order to avoid numerical dispersion, making the simulation as accurate and consistent as possible. The numeric control variables are DEPS, DCDIF, DCON, DCOFF, TOLE and LOGDIF, which determine how many new nodes are added and removed during adaptive gridding. These variables are described in Birkholzer and Karazaki, 1996 [3]. Determining optimal values for these variables has a strong effect on the accuracy, efficiency, and total length 
of program execution. Thus, these numerical parameters are adapted for each different main problem and maintained at those values for subsequent problem variations. Table 4 summarizes the different values used during different tests.

Next, input files elmt.inp and node.inp are also mandatory and must be supplied for any simulation. These files, coming from RENUMN, determine the structure of the mesh for the fracture network. The well node is chosen as close as possible to the center of the modeled field. Moreover, some other optional input files can be supplied: npn.inp belongs to this category and is used to obtain head values versus time for any desired node, such as the well node.

Last of all, we could consider the possibility of adapting ctrl.inp for each particular case we consider. Indeed, after some simulations, we found that customizing ctrl.inp did improve some mass-balance results. But (1) customizing raises the question of lengthy research times for each test, and (2) it would make it difficult to fairly compare physical results for different cases. Thus, to maintain a fair basis for comparison, once all the parameters are chosen for a base case, they are not modified thereafter. Table 5 reviews all the other parameters presented in the TRIPOLY section.

\section{II.3. Problems considered}

Once all the parameters have been specified and the sequence of the SWIW test is well defined, we need to choose a base case for which the parameter sensitivity study will be conducted.

\subsection{Large problem}

Based on some previous studies (Doughty and Tsang, 2009 [5]; Tsang and Doughty, 2009 [17]) and some actual field experiments conducted in Sweden, a first simulation problem has been established with the goal of creating a tracer plume with a typical spatial distribution and obtaining easily interpretable breakthrough curves. Thus, a "standard" procedure would consist of $1 \mathrm{~h}$ for the injection period, $5 \mathrm{~h}$ for the chase fluid period, and $500 \mathrm{~h}$ for the withdrawal period, with a flow rate equal to $q_{I}=2.8 \cdot 10^{-6} \mathrm{~m}^{3} \cdot \mathrm{s}^{-1}$ per meter in the $3^{\text {rd }}$ dimension which means that a total $10.08 \mathrm{~L}$ of tracer fluid are injected into the system (again per meter in the $3^{\text {rd }}$ dimension). The entire sequence of our modeled SWIW test would be quite similar to procedures followed for actual SWIW tests.

\subsection{Medium problem}

The injection-chase-withdrawal sequence described above turned out to be problematic, primarily due to the fact that there is a non-negligible loss of tracer through the outer 
boundaries of the model; this issue will be further described below in Section III.1.3. One probable explanation is a too long chase-fluid period. As a remedy, we design a medium problem with a shortened chase fluid period of 1 hour. The injection and withdrawal period remain unchanged, at 1 hour and 500 hours, respectively.

\subsection{Small problem}

Another possibility is that too large a volume of tracer was introduced into the system. As a remedy, we design a small problem, featuring a 30-minute injection time at the same flow rate as chosen before, 45 minutes for the chase fluid period, and $350 \mathrm{~h}$ of withdrawal. This small problem introduces only 5.04 L (per meter in the $3^{\text {rd }}$ dimension) of tracer in the system.

Table 6 displays a summary of the various problems considered.

\subsection{Parameter sensitivity study and hydrofracking}

The parameter sensitivity study is conducted to observe the system response to some particular effects. It includes a variation of three main physical properties of the rock matrix that can strongly change the solute exchange between the fracture network and the surrounding rock matrix: (1) matrix porosity $\Phi^{M}$, (2) diffusion coefficient $D_{e}$ and (3) retardation coefficient $R$. These parameters and their significance will be described in more detail below.

Hydraulic fracturing, or hydrofracking, may result in the creation of new rock fractures or an increase in aperture of existing fractures. We examine these two hydrofracking effects separately in our modeling study. For simplification, we assume that hydrofracking has similar effects in the entire model domain, not just near the injection well. Note that because this model of hydrofracking is so simple it should be considered as a preliminary approach in order to gain insight into the effect of the hydrofracking process on SWIWtest results.

\section{II.4. Displaying results}

\subsection{Population of matrix blocks}

We would like to examine how FMGN generates the fracture network and the subsequent distribution of matrix block size. Some minor revisions to POLY need to be performed to extract this particular information. The desired variable, called $S$, is the value of the maximum orthogonal distance of points in the matrix blocks to the adjacent fractures; in other words, this variable stands for the maximum existing distance from adjacent 
fracture to the considered block center. This datum provides us with a useful estimate for the different sizes of our numerous matrix blocks.

First, we create a histogram from the obtained list of S values, which can be broken down into three main groups: the small ones, with a distance lower than $0.001 \mathrm{~m}$; the medium ones, with a distance between $0.001 \mathrm{~m}$ and $0.05 \mathrm{~m}$; and the large ones, with a distance greater than $0.05 \mathrm{~m}$. Figure 8 shows the distribution of $\mathrm{S}$ values for the matrix blocks in the fracture-network geometry shown in Figure 5. It is evident that our study involves a densely fractured domain with small fracture distance and generally small matrix block sizes.

\subsection{What you see in the field: breakthrough curve and head change}

The breakthrough curve (BTC) is a line graph representing the evolution of the relative concentration at the well node. The relative concentration in the well is plotted versus the time of the experiment in seconds. A logarithmic scale is used for both of the two axes, to better emphasize some specific trends. The BTC can be divided into three main parts, which correspond to the same parts of the SWIW test: injection, chase fluid, and withdrawal. A linear scale for both of the two axes can also be used to better underline the beginning of the withdrawal part, especially when some comparisons are established.

Figure 9 exhibits a typical example of a tracer breakthrough curve as described above. Concentrations at the injection well increase during the injection period, followed by a strong decrease during the chase period, as pure water is injected. Withdrawal of water then leads to a temporary increase in concentrations, as contaminated water mostly from the fractures is being pulled back. This early increase during the withdrawal period is followed by a long tail of slowly decreasing concentrations (as solutes diffuse slowly out of the matrix blocks and are transported back to the well). We often superpose several BTCs together in the same graph in order to make comparisons more easily: in that case, the base case is usually represented in blue and the comparison is either in red, black, or green. Note that in SWIW tests in the field, the only observable portion of a breakthrough curve is during withdrawal, as water is removed from the system and tracer concentration can be measured. We show here the concentrations in the injection node for all time periods to provide additional information from the model, but we need to remember that BTC data for injection and the chase period are not available in field experiments.

The change in the flow field is exemplified by the head value at the well node. The magnitude of the head change to a given injection rate is an indicator of the permeability of the fracture network near the well.

\subsection{What we know from the model: spatial distribution and mass balance}

The spatial distribution in Figure 10 represents the relative concentration in our modeled fracture-matrix complex system at a given time. For the sake of visualization, the figure 
shows the average concentration value for each matrix block (averaged over the onedimensional variation within each block), the concentration between two nodes for the fracture network, and the calculated concentration value for each node. The range of relative concentration has a relatively large magnitude and is therefore plotted on a logarithmic scale. Both matrix blocks and fracture network segments are represented in the visualization, together with the considered node for the well. Figure 10 provides a typical example of the spatial distribution. We can see in Figure 10, which plots the situation at the end of the tracer injection period, that the fractures are the main transport pathways in the system, with concentrations that are typically higher than those in adjacent matrix blocks. Smaller blocks have higher average concentrations than larger ones. Typically, four snapshots of the system are presented together to give a better idea of the evolution of the spatial concentration distribution during the whole process of the SWIW test.

Another model output is mass balance, a useful tool to determine whether SWIW tests have a good recovery ratio. As already mentioned above, the adapted code yields values of the total tracer mass present in fractures and matrix blocks. Thus, the interesting values are (1) the total tracer mass at the end of the injection part, (2) the total tracer mass at the end of the chase fluid period and (3) the total remaining tracer mass in the system at the end of the withdrawal period.

However, the program has some difficulty with calculating the mass balance, and therefore our results must not be considered as absolutely correct, but rather as providing a tolerable estimate. Specific problems include a substantial sensitivity to time-stepping and an overestimate of the amount of injected tracer at the end of the chase-fluid period.

Meanwhile, some other calculations can be carried out to determine (1) the total retrieved mass at the well and (2) the total amount of tracer lost at the different sides during the injection and chase-fluid periods. Calculations are based on the fact that the flow at each side is known (the values are provided by an output file), as well as the average concentration for each time step. Thus, the mass of tracer retrieved at the well node, and likewise the mass of tracer lost at each side, can be known for each bin time with the following formula:

$$
M_{i}=\left(t_{i}-t_{i-1}\right) \cdot q_{\text {side }} \cdot \frac{C_{i}+C_{i-1}}{2} \cdot 1000
$$

where $M$ is mass in $g$ (per meter thickness in the $3^{\text {rd }}$ dimension) $t$ is time in $s, q_{\text {side }}$ is flow at the considered side in $\mathrm{m}^{3} . \mathrm{s}^{-1}$ (per meter thickness in the $3^{\text {rd }}$ dimension), $C$ is relative concentration in g. $\mathrm{L}^{-1}$, the factor of 1000 converts $L$ to $\mathrm{m}^{3}$, and the subscript $i$ stands for the bin time.

At the end of the withdrawal period, the Tracer Recovery Factor $r$ is calculated as follows:

$$
r=\frac{M_{\text {retrieved }}}{M_{\text {theoretical injected }}}
$$


This recovery factor is related to what we would measure in the field and tells us how important losses are through the outer boundary, while also giving an idea of how much tracer remains in the system at the end of the withdrawal period.

Similarly, we can also define another ratio called Mass Balance, which is just a check on the accuracy of the simulation, by the following formula:

$$
M B=\frac{M_{\text {retrieved }}+M_{\text {remaining }}+M_{\text {loss out side }}}{M_{\text {injected by program }}}
$$

With all these parameters finally set up, we can consider the first step of the project to be done. The second step, which is presented next, concerns results coming from some parameter sensitivity studies, where porosity $\Phi^{M}$, diffusion coefficient $D_{e}$ and retardation factor $R$ will vary within a predetermined range of values. Eventually a third step will address the effects of hydrofracking on our system, by increasing the fracture aperture and adding a third set of fracture to the two previous ones. 


\section{Results}

The next step of the project presents the results obtained after all the code adjustments are made and all the parameters are established beforehand. Detailed interpretations of the results are then presented in the parameter sensitivity study.

\section{III.1. Base cases}

In this part, we provide the results obtained after conducting the simulations described earlier, by showing histograms of block size distribution, breakthrough curves and spatial distributions of concentration for each case. Please refer to Figure 5 for the geometry of the fracture network considered.

\subsection{Histogram of block size distribution}

Figure 8 shows the histogram of block size distribution for our predetermined geometry as described in Section II.4.1. Note that the notion of "block size" is defined by variable $S$, which is the maximum orthogonal distance from the adjacent fracture to the considered block center. As illustrated by this diagram, almost 50\% of the blocks have block sizes ranging from $0.01 \mathrm{~m}$ to $0.05 \mathrm{~m}$, which is in accordance with the average block size value of $0.042 \mathrm{~m}$. Only about $1 \%$ of all blocks are larger than $0.25 \mathrm{~m}$.

These results clearly indicate that the fracture network generated for this example is very dense and creates mostly small matrix blocks. There are some larger blocks that could act as a semi-infinite matrix, and some very small blocks that could be filled up relatively quickly with solutes diffusing in from the fractures.

\subsection{Breakthrough curves}

Figures 11, 12, and 13 illustrate respectively breakthrough curves for the large problem, the medium problem, and the small problem. Each breakthrough curve comprises the evolution of solute breakthrough for the three different time periods previously defined for a SWIW test in Section II.4.2. Later on, we will focus only on the withdrawal period, which always starts with a peak and then gradually decreases until the end of the test. It should be noted that the BTCs all follow the same trend and present the same general characteristics.

Since the general behavior of the BTCs during the withdrawal period usually shows two linear sections (in the double log plot), a linear fit is added to the relevant part of the tail when possible. Figure 14 illustrates this situation, with Figure 15 providing a superposition of the three BTC into the same graph. From the three test cases shown in 
Figures 11, 12, and 13, we are interested in selecting an "optimal" base case, with criteria for optimal representation including (1) a sufficiently large number of blocks with different sizes affected by contamination, and (2) the injected tracer remaining in the modeled area.

\subsection{Spatial distribution and mass balance}

Figures 16, 17, and 18 show the respective spatial distributions of concentration in fractures and matrix blocks during SWIW tests for each problem in the following order: large, medium, and small problem. The distribution consists of four snapshots taken at (1) the end of the injection, (2) the end of the chase fluid period, (3) the middle of the withdrawal period and (4) the end of the withdrawal period. The figures nicely illustrate the transport processes occurring through the test sequence. At the end of injection, the fractures near the injection location exhibit the highest concentrations close to 1.0. As the advective-dispersive transport in the fractures away from the injection well is fast, solutes diffuse in a slower process into the porous matrix blocks. At the end of the chase period, with clean water being injected, fracture concentration decreases. This induces a reverse concentration gradient from the initially contaminated matrix blocks back to the fractures, but the diffusive exchange caused by this is relatively slow. Thus matrix blocks remain contaminated at the end of the chase fluid period. Withdrawal of water then pulls the remaining solute present in the model domain back towards the well, initially from the fractures in a fast process, then from the matrix blocks in a slow process. The concentration distribution shown at the end of withdrawal still shows very low concentrations of tracer present in the matrix blocks, with lowest values observed for the smallest matrix blocks. Table 7 gives mass-balance results for each case.

It is obvious that according to the spatial distribution and mass-balance results, the large problem case is not appropriate for our study. Despite it being the closest in testing sequence to actual field experiments, we are losing too much tracer on each side of the model domain, and the plume is too spread out, so that the recovery ratio would not be good enough. The small problem, on the other hand, shows a contaminant plume that does not extend very far from the injection point, implying that not many different block sizes are encountered.

For these reasons, we decided to use the medium problem as the base case for our various sensitivity studies as a reasonable compromise between the other two cases. To recap the conditions of the medium problem, it consists of injecting a tracer for one hour, then adding water to disperse this tracer for another hour and finally recovering as much tracer as possible over a long time period of 500 hours. The total length of our experiment in the field would be close to 21 days. 


\subsection{Tabulation of BTC characteristics}

When parameters such as porosity $\Phi^{M}$ or diffusion coefficient $D_{e}$ vary in a sensitivity study, two analyses may be conducted to compare results: (1) a spatial distribution analysis and (2) a breakthrough curve study. The former gives us an idea of the tracer advection and dispersion patterns influenced by matrix diffusion, while the latter must be examined carefully to determine whether the main characteristics of the case would be observable in the field.

Once all tests and analyses are conducted, we may determine three main features of the withdrawal period in the breakthrough curves on which to base our comparisons: (1) the peak concentration and time during that period, (2) the slopes of different portions of the tail, and (3) the length of the superposition between the linear fit and the curve for the tail. Two other main features could also be studied - the general aspect of the withdrawal period or the intersection time of the linear fits to the tail. However, they are strongly linked to the three previous features already presented.

\section{III.2. First parameter sensitivity study: porosity}

\subsection{Definition}

Used in hydrogeology, the porosity $\Phi^{M}$ of a porous medium such as a matrix describes the fraction of void space in the material, where the void may contain a fluid. However, in solute transport problems, we use the term effective porosity, which refers to the fraction of total volume in which fluid flow is effectively taking place, excluding deadends or unconnected cavities. Thus, matrix porosity is defined by the following ratio:

$$
\Phi^{M}=\frac{V_{V}}{V_{T}}
$$

where $V_{V}$ is the volume of void space where flow occurs and $V_{T}$ the total volume of the material, including the solid and all void components. It is therefore a function between 0 and 1 (or expressed as a percentage, $0-100 \%$ ).

The range of values considered in our case will be between $2 \%$ and $20 \%$, where the former could describe solid granite and the latter could depict sedimentary rock. The base case porosity value is $10 \%$. Low porosity problems are carried out within the range of $2 \%$ to $6 \%$; high porosity problems are carried out within the range of $15 \%$ to $20 \%$. Figure 19 illustrates the breakthrough curves for low and high porosity compared to the base case. Figure 20 represents the evolution of the spatial distribution during the SWIW test. Finally, Table 8 supplies a summary of BTC characteristics for the different conducted 
tests. Note that porosity in fractures does not vary during this sensitivity study, but porosity within matrix blocks does.

\subsection{Low porosity}

Typically, low porosity, between $2 \%$ and $6 \%$ in our study, would indicate that our considered material does not contain much void space. Thus, as this particular system is not very porous (e.g., solid granite), the injected tracer does not have much opportunity to diffuse into the matrix blocks, so there is much less tracer buildup in the matrix blocks. As a result, the tracer advects farther into the fracture network, exposing more rock matrix to tracer. Although there is little diffusion into each matrix block, a large volume of rock matrix is contacted by tracer, ultimately resulting in a significant amount of matrix diffusion.

This theory is illustrated by Figures 19 and 20. In the spatial distribution (Figure 20), the lower the porosity, the farther the contamination moves out from the injection well during the injection period. In other words, less tracer transfers into the matrix blocks, so that it can be transported to more places in the fracture network. As a result, the plume is generally enlarged. Therefore, as more tracer advects through the fracture network, there are correspondingly more matrix blocks contacted by tracer. The usefulness of the chasefluid period, namely introducing water such that tracer contacts more matrix blocks, is also highlighted by these results. In addition, the concentration of the closest matrix blocks to the well is higher than in the base case. Since the porosity is lower, the matrix blocks have less ability to receive and store tracer, which results in higher fracture and higher matrix concentrations near the injection well.

The general behavior of the system is also different at the end of the withdrawal period. The plume is still quite spread out, because it takes a long time to recover the tracer stored in the matrix blocks. In other words, as more matrix blocks are contaminated by the tracer, it is normal to still observe many matrix blocks remaining well saturated, because diffusion is a much slower process than advection. Moreover, it should be noted that there is very little remaining tracer present in the fracture network, and some small blocks are also completely empty: all solutes have been recovered, owing to advection transport and the diffusive effect for the small blocks.

Regarding the BTC characteristics as shown in Figure 19 and characterized in Table 8, at the beginning of the withdrawal period they are also evolving as expected. The peak height is higher than in the base case because more tracer is present in the fracture network, where it can be readily withdrawn. The peak height occurs slightly later than before, because it takes slightly longer to recover tracer from the more distant regions of the plume in the fracture network. Note also that the slope of the first part of the tail is almost a $-3 / 2 \log$ slope, which is characteristic of tracer recovery from a semi-infinite medium, but this is not the case for the second part of the tail, which seems surprising. 
Concentrating on the late-time behavior, we note that the late-time slope is more negative than in the base case, indicating that there is less tailing than with higher porosity values. In fact, once the tracer located in the fracture network has been retrieved and the fracture concentrations are all very low, the recovery of the tracer located in the matrix blocks takes more time and leads to low concentrations and less tracer recovered. Hence, it is to be expected to retrieve less tracer at the end if we retrieve more tracer at the beginning. Besides, the length of the linear fit is quite long and the slope of the second tail is more negative than the $-3 / 2 \log$ slope, indicating that finite matrix block effects are significant. That is, the tracer that was in the finite matrix block has already come out, so the BTC decreases faster. It is, however, also possible that the lowest-porosity BTC shows a single linear dependence instead of two different linear parts.

\subsection{High porosity}

Conversely, a high porosity (20\%) such as is typical of sedimentary rock would indicate ample opportunity for diffusion and solute storage into the rock matrix. The opposite effects (compared to low porosity rock) are therefore observed: much of the injected tracer diffuses into the matrix, so that tracer can more easily fill the closest matrix blocks to the well. As a consequence, the tracer does not extend very far from the injection well, as is reflected in Figures 19 and 20. According to the spatial distribution, the higher the porosity, the smaller is the plume, implying that fewer matrix blocks are contaminated, but that there is more diffusion into and solute storage in those fewer matrix blocks.

Regarding the BTC characteristics shown in Table 8, the peak height is also lower than in the base case, for all the reasons mentioned earlier. The peak time occurs slightly earlier than in the base case, because there is more tracer located near the well, which can be quickly recovered when the withdrawal period starts. As a result, the length of the linear fit is longer than in the base case. The late-time behavior of our system is also modified when porosity is increased. We can still observe through the spatial distribution that the plume is concentrated near the injection point, so that fewer matrix blocks than in the base case are playing a role. However, it should be stressed that the remaining mass in our system is still quite significant. The spatial distribution at the end of withdrawal shows us that there is still a substantial amount of tracer inside the fracture network (as opposed to the low porosity case), because the matrix blocks are still feeding the fracture network with tracer.

Concerning the BTC, the late time tail is higher than the base case, mainly because of the remaining mass still present in our system. The slope of the second part of the tail and the linear fit are also similar to the base case. However, increasing porosity has two opposite effects: it implies more diffusion, but also more capacity for the matrix to hold tracer. Finally, it should be noted that the effects of lower porosity on the spatial distribution and the BTCs are more visible in the system than the effects of higher porosity. 


\section{III.3. Second parameter sensitivity study: diffusion coefficient}

\subsection{Definition}

The diffusion coefficient (or diffusivity) is a proportionality constant between the molar flux due to molecular diffusion and the gradient of concentration for the considered species. In our case, the considered diffusion coefficient is called "effective diffusion coefficient", or $D_{e}$, which describes diffusion through the pore space of porous media. Molecular diffusion in matrix blocks is given as follows:

$$
D_{e}=D_{f w} \cdot \Phi^{M} \cdot \tau
$$

where $D_{f w}$ is the diffusion coefficient in the water filling the pores (and which is defined in Section II.2.3), $\Phi^{M}$ is matrix porosity, and $\tau$ is tortuosity, a factor that is difficult to estimate. Because we have considered porosity variation in the previous section, when we talk about variation in the effective diffusion coefficient, we are essentially referring to the variability of tortuosity $\tau$, which is a fitting parameter. The physical effect of the diffusion coefficient is such that the higher the diffusivity (of one substance with respect to another one), the faster they diffuse into each other.

For our chosen base case, the matrix blocks in our domain have a diffusion coefficient of $2 \cdot 10^{-9} \mathrm{~m}^{2} \cdot \mathrm{s}^{-1}$. The range of values for this diffusion coefficient starts from $4 \cdot 10^{-10} \mathrm{~m}^{2} \cdot \mathrm{s}^{-1}$, which corresponds to the base case value divided by five, hence considered as the small diffusion effect. Large diffusion effects are comprised between $4 \cdot 10^{-9}$ and $2 \cdot 10^{-8} \mathrm{~m}^{2} . \mathrm{s}^{-1}$, i.e., the base case value is respectively doubled and multiplied by five.

Figure 21 illustrates the respective breakthrough curves for small and large effective molecular diffusion coefficients compared to the base case. Figure 22 represents the evolution of the spatial distribution during the SWIW test. Finally, Table 9 recaps BTC characteristics for the different tests.

\subsection{Small diffusion}

The base case has a diffusion coefficient equal to $2 \cdot 10^{-9} \mathrm{~m}^{2} \cdot \mathrm{s}^{-1}$. The first sensitivity study divides this value by five. Like decreasing porosity, decreasing the diffusion coefficient serves to limit the amount of solute taken up by the rock matrix. However, unlike decreasing porosity, when the diffusion coefficient is decreased, the capacity of the matrix to hold tracer remains unchanged. Consequently, the effects shown are similar to those previously observed for the porosity study, but with some noticeable differences in matrix saturations, as exhibited by Figures 21, 22, and Table 9 .

Concerning the spatial distribution, the plume, while still well spread out, is a bit less so than for the low porosity case, because the matrix blocks are less prone to filling up with solute. Basic characteristics, such as an enlarged plume with more tracer transported in 
the fracture network and more matrix blocks affected, are noted. Moreover, the concentration of the closest matrix blocks to the well is a bit lower than in the base case. At the end of the withdrawal period, the same general pattern for the plume can be observed, namely a low-concentration tracer plume in the matrix blocks still well dispersed, and virtually no tracer present in the fracture network.

Regarding the BTC, the previous discussion for low porosity is almost entirely applicable for diffusion coefficient. The main differences reside in (1) the magnitude for the peak height and time, which is a little bit lower for the diffusion BTC, (2) the matrix blocks, which do not fill up so fast, (3) more blocks still filled up with tracer (especially the small blocks) and (4) the first part of the tail, which does not have a $-3 / 2 \log$ slope. However, it turns out that the second part of the tail has "nearly" a $-3 / 2$ slope: our finite matrix blocks act now more like infinite ones, because of the higher storage capacity in the matrix. Besides, the first part of the tail has a stronger slope than the base case, as with low porosity, but the general aspect is much more concave than in the low porosity case. For this particular case, it is impossible to model any part of the BTC with a linear fit.

\subsection{Large diffusion}

A large diffusion coefficient, such as the base-case value multiplied by ten, indicates that diffusion from the fractures into the surrounding matrix blocks is more effective. Hence, increasing the diffusion coefficient is "similar" to increasing porosity but without increasing storage capacity. According to the spatial distribution shown in Figure 22, there is indeed less tracer in the fracture network, but the concentration in the matrix blocks nearest to the well is also higher than the base case. So when diffusivity is increased, more tracer is going into the matrix blocks, owing to this diffusive effect. As a result, there is also less tracer in the fracture network, just as was observed for the increased-porosity case. Given that diffusion is a process that takes time, the nearest fractures to the well center are still contaminated by the tracer coming back from the matrix blocks.

Focusing on the BTC represented by Figure 21, we find the general behavior quite similar to high porosity, namely, the peak height lower than in the base case and the late time tail higher than in the base case. But when we look at some BTC characteristics, some differences appear, resulting from the diffusive effect. First, the retrieved mass is higher than in the base case coupled with a remaining mass lower than in the base case: the recovery ratio is very good. Second, there is a "long" transition between the first and the second parts of the tail, such that the general aspect is more concave and the lengths of the linear fits are shorter than usual. As a consequence, the slope of the second part of the tail is more negative than usual: the effects of finite matrix blocks are considerable in this particular case.

The differences in BTCs (Figure 23) for the high porosity and large diffusion cases may arise from the fact that although diffusion into the rock matrix is increased for both cases, only for the high porosity case is the capacity of the matrix blocks to hold tracer 
increased. Thus, more blocks (especially the smaller matrix blocks) may become saturated with tracer for the large diffusion case. During the withdrawal period, recovery of tracer from saturated blocks differs significantly from recovery of tracer from unsaturated blocks, which act like a semi-infinite medium. We interpret departures from a linear tail with a $-3 / 2$ slope as indications that finite matrix block effects are important.

\section{III.4. Third parameter sensitivity study: retardation coefficient}

The retardation coefficient $R$ was previously defined in this report (see Section II.2.2., Equation 12) and is related to sorption effects. We know that sorption tends to slow the effective transport velocity of contaminants dissolved in groundwater. When this effective transport velocity is less than the pore water velocity of the groundwater, the contaminant is said to be retarded. The retardation coefficient $R$ is used to estimate this retarded contaminant velocity. It is a dimensionless number greater than or equal to 1 .

Our base case involves $R=1$, which means that sorption is not taken into account. In the sensitivity study, different values of retardation are used with values up to $R=25$. Figure 23 illustrates the breakthrough curves for different values of retardation coefficient compared to the base case. Figure 24 represents the evolution of the spatial distribution during the SWIW test. Finally, Table 10 summarizes the BTC characteristics for each test. However, we should point out that the retardation effects on our system are somewhat uncertain, because the simulator does not account for sorbed mass when calculating mass balance values, and thus the mass balance of sorption runs cannot be adequately checked.

A higher retardation coefficient enhances the storage capacity in matrix blocks, as solutes can be sorbed onto rock grain surfaces in addition to be dissolved in the water. The spatial distribution of solute concentration in the model domain (Figure 24) shows at first sight the expected behavior of our system when subject to retardation. Specifically, when retardation increases, the plume is less spread out, and diffusion into distant matrix blocks is less significant. But it turns out that the concentration in the blocks nearest to the well is lower, as is the concentration in the fracture network. In fact, a large retardation factor implies substantial capacity to uptake tracer in the matrix blocks: the diffusive effect is now coupled with the sorption effect. Hence the matrix blocks are first "consuming" the tracer, due to sorption, and then storing it, due to diffusion. This explanation also applies to the spatial distribution at the end of the withdrawal period. The process of pulling tracer back from matrix blocks is very long, because of the matrix's capacity to store tracer, especially when retardation becomes more and more important. That is why there is still a lot of tracer in the fracture network. We can therefore suppose that there is a non-negligible remaining mass in our retarded system, and that it takes more and more time to retrieve the injected tracer when sorption is relevant. 
The BTCs (Figure 23) for retardation cases generally illustrate these same processes: for instance, the peak height is lower than in the base case. However, some other findings can also be deduced strictly from the BTC. First of all, the first part of the tail is quite long, which means that the tracer is coming back from finite matrix blocks during much of the withdrawal period. As a direct consequence, the length of the second linear fit is quite short, but it has a $-3 / 2$ slope, which is the signature of tracer coming back into the fractures from semi-infinite matrix blocks.

\section{III.5. Hydrofracking effects}

Hydrofracking can have two main consequences both increasing the permeability of the rock mass: either increasing the fracture aperture of existing fractures or creating new fractures. The second consequence can be represented by adding a third fracture set to the initial model. While we evaluate here the effect of hydrofracking on the observed SWIW test response, we should point out that this study is somewhat academic: our fracture network is quite well connected and permeable, which means that hydrofracking would not be necessary in this case.

\subsection{First effect: larger fracture aperture}

Hydrofracking can lead to an increase in fracture aperture. We are interested in evaluating the lower limit of aperture increase at which we can observe noticeable changes in BTC or spatial distribution. We study three different size effects (small, medium, and large) in order to understand the required scale for those changes.

After some trials, it appears that there is only a small effect on tracer transport when the aperture is doubled, i.e., when $2 b=2 \cdot 10^{-4} \mathrm{~m}$. Thus, an increase of $25 \%, 50 \%$ or even $75 \%$ of the fracture aperture can be considered as "negligible" for us if we look only at the BTC. However, we should not forget the pressure change: small increases in aperture lead to significantly reduced pressure increases in response to injection. Table 11 recaps the different pressure increases for each aperture modification, in addition to the various BTC characteristics. Regarding the other cases, medium and large effects are obtained when the aperture is multiplied by factors of five and ten, respectively. What we should expect from this change is that when we increase aperture, (1) diffusion becomes a smaller effect compared to advection, and (2) there is less fracture-matrix contact area relative to fracture volume. Figure 25 shows the breakthrough curves for the small, medium, and large aperture changes in the same plot, and Figure 26 exhibits the spatial distribution for these cases.

If we first focus on the spatial distribution as represented in Figure 26, we can see that the plume is less spread out for larger aperture cases. By increasing the aperture while keeping the same flow rate of injection, there is more tracer staying in the fractures. Hence, as the aperture increases, the fracture network close to the well contains more 
tracer, and the nearest blocks to the well are slightly more contaminated. However, at the end of the withdrawal period, the various cases show a similar pattern: the fracture network is empty and the blocks still contain some tracer. This results from advective transport being easier to reverse and being a faster process than diffusion, so the influence of increasing aperture is less visible at the end of the withdrawal period.

Moreover, what we know from the model (spatial distribution) is in accordance with what we could see in the field, namely the BTC (Figure 25) and the pressure change as shown in Table 11. The peak concentration during the withdrawal period varies almost proportionally upwards with increasing aperture, and the peak time occurs proportionally later. Concerning the slope of the tails, the second part is similar to the base case, whereas the first part has a more negative slope compared to base case. Note that the general behavior of BTCs is in accordance with the proposed theory, namely more recovered tracer at the beginning, because of the faster advection and more tracer stored in the fracture network. However, we are not able to observe any $-3 / 2$ slope for the second part of the tail, so the blocks still have to be considered as a finite medium. Finally, the pressure change reduces strongly when the aperture is increased.

\subsection{Second effect: more fractures in the field}

Hydrofracking can also create a new set of fractures. This effect is investigated here by adding a third set to the two previous ones, with a predetermined amount of fractures in the third set. The number of additional fractures is limited by some numerical issues, namely the length of program execution and stability regarding the geometrical choice. Nevertheless, we were able to insert a third set with the same number of fractures as the previous ones ( 700 fractures per set). We also run two other cases adding a third set including 200 and 450 fractures, respectively. Table 12 provides the different pressure values obtained for each numerical experiment, as well as the diverse BTC characteristics. Figure 27 displays the new block size distributions when adding new fracture sets with 200, 450, and 700 fractures. Figure 28 shows the BTC, while Figure 29 illustrates the spatial distribution when a third set of fractures is added.

Figure 27 provides the various histograms for each new third set, ranked in ascending order by the number of fractures, and shows the comparison between the different cases. At first glance, there is little difference, in part due to the fact that the original fracture network was already quite dense and well connected, so the effect of adding fractures is relatively modest. On average, the matrix blocks are slightly smaller when adding more fractures, so they will act less infinite. If we now focus on the spatial distribution as represented by Figure 29, we realize that there are virtually no major modifications or changes compared to the base case. The geometry is obviously different, but the characteristics remain similar: fractures well filled up with tracer, matrix blocks near the well easily filled up, and plumes almost the same size. Nevertheless, the tracer extent for the 700-fractures case is a little bit smaller than the others. Concerning the end of withdrawal, the same general pattern is observed between the cases. 
The BTC confirms the similarity between all cases: the peak height and time are of the same magnitude for all the cases. The only anomalous part resides in the nonproportional increase of the peak height versus the number of fractures added. But as shown by Table 12, this could be linked to a numerical mistake when adding too many fractures, because of the problems encountered with the remaining mass calculation. The other noticeable change can be found in the late-time part of the withdrawal: the larger the number of fractures, the more the late-time tail of the BTC departs from a linear fit with a $-3 / 2$ slope. Thus, the change in block size distribution that comes from the addition of new fractures makes for fewer matrix blocks acting infinite.

Actually, a theory explaining the reason why adding more fractures has so little effect on BTC can be expressed. By adding more fractures, the contact area between the fracture network containing tracer and the matrix blocks is not necessarily increased: there is just a change in the spatial distribution of tracer-filled fractures. In other words, if there are more fractures near the well, the tracer does not travel far away from the well, but it still contacts approximately the same amount of matrix (unlike an aperture increase, where there is less fracture-matrix contact area). There is a difference in the relative importance of diffusion and advection, since advection through any one fracture is slower when there are more fractures.

Finally, it is also possible to observe a non-negligible pressure change when more fractures are added to the field, as illustrated by Table 12. Indeed, as the total number of fractures is increased (i.e., the fracture network is larger than the base case), the overall permeability of the fracture network is larger. However, this pressure change is less significant than when increasing fracture apertures, because of the proportionality of the pressure gradient to the cubed fracture aperture.

\subsection{Comparison between the hydrofracking cases}

To objectively compare the sensitivity between additional fractures and enlarging the aperture, we must establish a fair comparison (i.e., the same additional fracture volume should be generated by either case). Thus, the ratio of fracture volume before and after hydrofracking is calculated. For the enlarging-aperture cases, it simply corresponds to the factor by which aperture is increased, whereas for the additional fracture cases, it is equal to the ratio of the new total volume of fractures in the model area over the total volume of fractures in the base case (values provided by RENUMN).

The increased-aperture cases that were first considered as having too small an effect on BTCs (refer to Section 5.1) are interesting here, because increasing aperture from $25 \%$ up to $75 \%$ changes fracture volume comparably to adding a third set of fractures, as illustrated by Table 13. Figure 30 represents the BTCs for this small aperture increase and can be compared to Figure 28 for adding a third fracture set. We notice that the peak concentrations during withdrawal are very similar for both hydrofracking cases. Regarding the late-time tails, they all are quite similar for the increasing-aperture cases, while they are all a bit different for the cases that add a third fracture set (but still 
relatively close to the base case). Generally speaking, the BTC results are globally the same between the two hydrofracking cases and the base case. In contrast, the pressure drops faster for the aperture-increasing cases than when adding a third set because of the cubic law, as already explained in Section 5.2.

Ultimately, this comparison makes us realize that our initial geometry is very dense (a highly connected fracture network), which could explain why adding a third set to our chosen geometry does not fundamentally change the system. It should also be stressed that when both hydrofracking changes are comparable, they behave in the same way in terms of breakthrough behavior, and only the pressure change can help us to distinguish them.

\section{III.6. Comparison between sensitivity cases}

After all the different sensitivity simulations are carried out, some combinations of results are made to determine which effects are the most significant compared to others. Pairs of tests involving two varying parameters allow us to determine that retardation has the strongest effect on the system, followed by porosity and then diffusion, as expected after the results obtained during the parameter sensitivity study.

Similarly, some comparisons with the base case are performed between analogous effects with respect to their BTCs, because it is hard to precisely distinguish key features in the spatial distribution. Actually, the spatial distribution only gives us general trends about a case, because there are no precise indicators that we can look at carefully, unlike breakthrough curves.

Three groups can be formed: (1) the group in which BTCs are higher than the base case at the beginning of the withdrawal period and lower at the end (indicating less retarding function of the matrix blocks), represented by Figures 31 and 32, which illustrate respectively the BTC and the spatial distribution; (2) the opposite, where BTCs are first lower and then higher (indicating more retarding function of the matrix blocks), illustrated by Figure 33 for the BTC and Figure 34 for the spatial distribution; and (3) the group in which there is virtually no difference with the base case, as represented by its BTC (Figure 35) and its spatial distribution (Figure 36).

As mentioned above, the first group corresponds to BTCs that are higher than the base case at the beginning of the withdrawal and lower at the end. The higher peak indicates that more tracer can be immediately retrieved, and it makes sense for the late-time slope to be more negative than the base case. It is therefore not surprising to gather in this category a low porosity, a small diffusion, and a bigger aperture case. Although these cases share the same BTC behavior in trend, as shown on Figure 31, this is not the case for their spatial distributions, as represented by Figure 32, of the outlier being the largeraperture case. 
The second group describes the opposite situation, i.e., BTCs are first lower and then higher than the base case. The group contains a high porosity, large diffusion and high retardation effect. According to what has been previously studied, it is logical to find these parameters together, given their resemblance to each other. According to Figures 33 and Figure 34, not only are they all presenting the same characteristics on the BTC, but they share similar spatial distributions as well.

Finally, the third group assembles the parameters that show no significant differences from the base case. They comprise a slightly higher porosity (15\%), a doubled aperture, and slightly more fractures added into the system (200 fractures). These effects are considered as small effects - they cannot be neglected but they have only a small influence on overall transport behavior. They are reflected in Figure 35 and (by their spatial distribution) in Figure 36. 


\section{Discussions}

This section discusses some issues concerning the numerical performance of TRIPOLY and some ideas that arise from the results.

\section{IV.1. Mass balance}

At this point, some comments must be made regarding mass-balance results. The first general point to stress is that the mass-balance calculation provided by the program is not completely accurate. For example, the value calculated by the program for the injected mass in the system is always slightly overestimated. Moreover, some issues exist with mass-balance results regarding the retrieved mass: it can be greater than the injected mass, as shown by Table 7. This problem occurs when too much tracer goes outside the domain of study, confirming that the code has some difficulties in treating this particular case. We also point out that some mass-balance results for the recovered mass seem substantial in error. The same problem arises for retardation, but we know that the code does not take into account retardation in the calculation.

Another issue that could be stressed is the dependence of the results to certain parameter choices driving the numerical solution process. The code is very sensitive to timestepping, and this too has a non-negligible impact on mass-balance results. Moreover, adding too many fractures to the system can lead to problems of consistency and reliability with some results: such "overloading" could affect the remaining mass calculation carried out by the code, as illustrated by Table 12 for the 700-fractures case. Given all this, clearly some improvements need to be made within this new feature of TRIPOLY.

\section{IV.2. Inverting BTC}

It is clear that the entire simulation study described in Section III is based on precisely knowing the physical settings used for each test, so that we can observe the response of the system to our various experiments. After running a series of simulations, we have at our disposal the predicted transport behavior for various sensitivity cases. In particular, we have determined BTCs, which are observable in the field. The question arises whether the process can be inverted, that is, would one be able to derive from the BTCs some relevant predictions with respect to fracture-matrix system parameters.

One thing to emphasize is that for a given BTC, there is more than one possible set of parameters required to reproduce it. The goal of this study is to examine carefully some specific BTC characteristics and then try to infer information about the corresponding parameters. Indeed, the information available for typical SWIW field experiments is the 
breakthrough curve and the pressure change. As already described earlier in Section III.1.4, the BTC characteristics that we are studying are the peak concentration and time during withdrawal, the slope of the tails, and the length of the superposition of the linear fit and the effective curve. Table 14 summarizes the results found regarding the correlation between physical settings and BTC characteristics.

Concerning the peak concentration, the higher the peak, the more tracer can be retrieved straightaway. This implies that the tracer is mainly contained in the fracture network or in the nearest surrounding matrix blocks. This is exactly the case for a low porosity, a small diffusion coefficient, and/or a large aperture. Not only do these properties share the same characteristic, but they have similar effects on BTC as well. In contrast, the smaller the peak, the more difficult it is to recover tracer once the withdrawal period has started. This can be explained by there being less tracer in the fracture network and more tracer penetrating into the matrix blocks, so it takes a while to reverse this process. As expected, this characteristic is shared by the elements of the second group, namely a high porosity, a large diffusion coefficient, and a significant retardation effect.

Regarding the $-3 / 2$ slope of the BTC tail, recall that it is the signature of tracer coming back into the fractures from a semi-infinite matrix. In other words, the biggest blocks of our system can sometimes act as semi-infinite for the time periods considered, even though we have generated only finite matrix blocks. A $-3 / 2$ log slope is therefore expected for the second part of the tail when we have high porosity, small diffusion, and small retardation. It would seem consistent that our finite matrix blocks act more infinite (1) when the diffusion coefficient is small, because not much tracer moves into the matrix blocks; (2) when retardation is large, because there is more capacity for storing tracer in the matrix; and (3) when porosity is large, because diffusion is more important, even though there is also more capacity.

Finally, with respect to the length of the linear fits for the BTC tail, generally, the longer the fitted portion, the more significant it is. Often, a long fitted portion is associated with a previous short fitted part, as described in Table 14. The problem is that it is a qualitative parameter and not a quantitative one. It also depends on how long the withdrawal period lasts, so that we would be able to observe longer tails, but with more uncertainty due to the limited precision of low-concentration measurements. We can observe that usually a short tail in the second part of the withdrawal curve can be linked to a $-3 / 2$ slope: the semi-infinite matrix block behavior takes time to appear, due to the presence of a range of matrix-block sizes in our model.

It is hard to precisely determine the possibility of inversely determining physical properties of the fracture-matrix system from the BTCs. There are many variables to take into account, and it is difficult to distinguish precisely some effects among others. However, we should not forget that SWIW-test BTCs can provide some general sense of the characteristics of fractured rocks, as well as providing constraints on the parameters relevant to tracer diffusion and sorption through fractured media. Besides, the results can be coupled with other tracer tests to establish some crosschecks between those tests that are focusing on different physical parameters. 


\section{IV.3. Comparison with temperature case}

Thermal SWIW tracer tests have recently been modeled to determine the fracture-matrix heat transfer area, as reported in Pruess and Doughty, 2010 [14]. Here, we would like to compare the consistency of our results with those results.

The general idea of the temperature case is to focus on SWIW tests in which temperature itself is used as a tracer, and then observe the thermal response to (1) making additional fractures accessible to injected fluids, which is similar to adding a third set of fractures, and (2) increasing the aperture and permeability of pre-existing fractures, which is similar to increasing aperture in our study. When aperture was decreased and the fracture-matrix contact area was concurrently increased, as in our aperture variation study, a larger matrix effect was observed, just as in our results. However, when a new fracture was added, a slightly larger matrix effect was noticed, whereas our simulations almost no effect on BTCs.

Ultimately, our results can be considered consistent with the temperature case, considering the very different relative importance of diffusion and advection for temperature and tracer problems. (For the temperature problem, the equivalent diffusion coefficient would be four orders of magnitude greater than for our base case.) Besides, the temperature case considers only a semi-infinite matrix, whereas we take into account a range of finite-matrix-blocks sizes, with a few larger matrix blocks probably acting semi-infinite. 


\section{Conclusions and future work}

An evaluation of the SWIW method has been carried out, using the numerical code TRIPOLY, for a complex fracture-matrix model consisting of a two-dimensional network of fractures surrounded by rock matrix blocks of various sizes and shapes. Results of modeling SWIW tests show that TRIPOLY can meet the requirements for such model experiments as long as some adjustments are made (TRIPOLY was indeed not designed for this specific purpose). Furthermore, we must be careful when choosing the numerical parameters that control accuracy and error tolerance within TRIPOLY. Ultimately, the solute transport modeling done by TRIPOLY is quite acceptable: numerical predictions for BTCs and spatial distributions are consistent with each other and with mass-balance calculations. Parameter sensitivity studies performed on matrix properties porosity, diffusion coefficient, and retardation coefficient show that the basic physical processes are well represented by our model, both in the fracture network and the matrix system. Potential changes created by hydrofracking are also modeled to observe the response of our model and compare the consistency of these results to similar previous studies. The results of all simulations confirm the importance of the role that the matrix plays in solute transport through fractured rock.

The modeling studies can be summarized in terms of how various parameter choices control the impact that the rock matrix has on tracer transport, and how this impact is manifested in SWIW-test tracer distributions and BTCs, as illustrated by Table 15. The spatial tracer distributions provide more insight into the physical processes that occur, but the BTCs (together with injection pressure) are all that we will typically be able to observe in a SWIW test under real field conditions. Hence, it is valuable to study the BTCs carefully, in order to infer as much as possible about the physical processes occurring for the various cases. Formally inverting SWIW-test BTCs could potentially be a very useful tool for scientists, allowing them to infer field-scale transport properties, information on fracture-network/matrix-block geometry, or the results of hydrofracking. As Table 15 indicates, BTCs do not provide unique solutions for these properties, but comparing different features of the BTCs do provide some indication of what properties or processes could be responsible. This suggests that SWIW-tests can be a valuable contribution to site characterization, especially if coupled with other forms of hydrologic and tracer testing.

Future work that could be done would consist of more precisely adapting the TRIPOLY code to various potential SWIW test designs, such as including a rest period. We could also vary the values for some currently unused parameters, such as introducing a nonzero standard deviation for fracture aperture or orientation. Even with the same parameters, it would be interesting to create a different geometry for the fracture network to see its impact on BTCs. It would be also interesting to consider other hydrofracking consequences such as increasing both aperture and number of fractures. Additionally, more realism could be put into the hydrofracking cases - for example, to consider aperture increase as a function of distance from the well. Another important follow-up 
possibility would be to generate a much sparser fracture network (still connected) for the base case; this would provide better tests of hydrofracking, because hydrofracking would significantly increase fracture connectivity. It could also be interesting to compare the results obtained with TRIPOLY to other codes, such as TOUGH. For this comparison, it would be useful to determine the optimal numerical parameter choices for TRIPOLY when using a tracer equivalent to temperature, which acts like a strongly sorbing tracer. 


\section{Acknowledgments}

The main author is very grateful for the internship carried out at Lawrence Berkeley National Laboratory and directed by Jens Birkholzer. He also would like to thank his school of engineering, INSA de Lyon, for the opportunity of doing a research internship. The main author particularly thanks Christine Doughty for our stimulating discussions, careful reviews, and helpful comments, and Jens Birkholzer for his technical support and general work supervision. Technical review by $\mathrm{Chin}-\mathrm{Fu}$ Tsang and editorial assistance by Dan Hawkes are also appreciated. This work was supported by INSA de Lyon and performed under the auspices of the U.S. Department of Energy and Lawrence Berkeley National Laboratory through Contract No. DE-AC02-05CH11231.

\section{References}

[1] Billaux, D., S. Bodea and J. Long, FMG, RENUM, LINEL, ELLFMG, ELLP and DIMES: Chains of programs for calculating and analyzing fluid through twodimensional fracture networks - Theory and design, Lawrence Berkeley Laboratory Report LBL-24914, Berkeley, CA, 1988.

[2] Billaux, D., J. Peterson, S. Bodea and J. Long, FMG, RENUM, LINEL, ELLFMG, ELLP and DIMES: Chain of programs for calculating and analyzing fluid through two-dimensional fracture networks - User's manual and listings, Lawrence Berkeley Laboratory report LBL-24915, Berkeley, CA, 1989.

[3] Birkholzer, J. and K. Karasaki, FMGN, RENUMN, POLY, TRIPOLY: Suite of programs for calculating and analyzing flow and transport in fracture networks embedded in porous matrix blocks, Rep. LBNL-39387, Lawrence Berkeley National Laboratory, Berkeley, CA, 1996.

[4] Doughty, C. and K. Karasaki, Flow and transport in hierarchically fractured rock, Journal of Hydrology 263, pp. 1-22, 2002.

[5] Doughty, C. and C.-F. Tsang, Analysis of three sets of SWIW tracer-test data using a two-population complex fracture model for matrix diffusion and sorption, Lawrence Berkeley National Laboratory, Berkeley, CA, 2009.

[6] Gouze, P., T. Le Borgne, R. Leprovost, G. Lods, T. Poidras and P.A. Pezard, NonFickian dispersion in porous media: 1. Multiscale measurements using single-well injection-withdrawal tracer tests, Water Resources Research 44, W064426, doi:10.129/2007WR006278, 2008.

[7] Haggerty, R., S.W. Fleming, L.C. Meigs and S.A. McKenna, Tracer tests in fractured dolomite. 2. Analysis of mass-transfer in single-well injection-withdrawal tests, Water Resources Research 37, no. 5, pp. 1129-1142, 2001.

[8] Karasaki, K., A new advection-dispersion code for calculating transport in fracture networks, Rep LBL-22090, Lawrence Berkeley National Laboratory, Berkeley, CA, 1986. 
[9] Najita, J. and C. Doughty, Using TRINET for simulating flow and transport in porous media, Rep. LBNL-42158, Lawrence Berkeley National Laboratory, Berkeley, CA, 1998.

[10] Neretnieks, I., Single Well Injection Withdrawal Tests (SWIW) in fractured rockSome aspects on interpretation, Report R-07-54, Department of Chemical Engineering and Technology, Royal Institute of Technology, Stockholm, Sweden, 2007.

[11] Nordqvist, R. and E. Gustafsson, Single-well injection-withdrawal tests (SWIW). Literature review and scoping calculations for homogeneous crystalline bedrock conditions, SKB R-02-34, Swedish Nuclear Fuel and Waste Management Co. (SKB), Stockholm, Sweden, 2002.

[12] Nordqvist, R. and E. Gustafsson, Single-well injection-withdrawal tests (SWIW). Investigation and evaluation aspects under heterogeneous crystalline bedrock conditions, SKB R-04-57, Swedish Nuclear Fuel and Waste Management Co. (SKB), Stockholm, Sweden, 2004.

[13] Pruess, K. and K. Karasaki, Proximity functions for modeling fluid and heat flow in reservoirs with stochastic fracture distribution, Proc. $8^{\text {th }}$ Workshop Geotherm. Res. Eng., Stanford: 219-224, 1982.

[14] Pruess, K. and C. Doughty, Thermal single-well injection-withdrawal tracer tests for determining fracture-matrix heat transfer area, Lawrence Berkeley National Laboratory, Berkeley, CA, 2010.

[15] Schroth, M.H., J.D. Istok and R. Haggerty, In situ evaluation of solute retardation using single-well push-pull test, Advances in Water Resources 24, pp. 105-117, 2001.

[16] Segan, S. and K. Karasaki, TRINET: A flow and transport code for fracture networks - User's manual and tutorial, Lawrence Berkeley National Laboratory annual report, Berkeley, CA, 1993.

[17] Tsang, C.-F. and C. Doughty, Insight from simulations of single-well injectionwithdrawal tracer tests on simple and complex fractures, Rep. LBNL-2487E, Lawrence Berkeley National Laboratory, Berkeley, CA, 2009. 


\section{List of Tables}

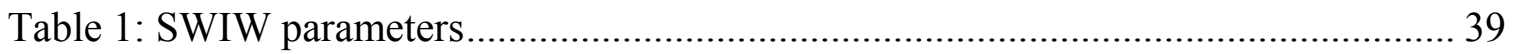

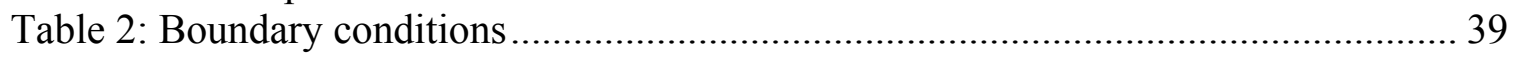

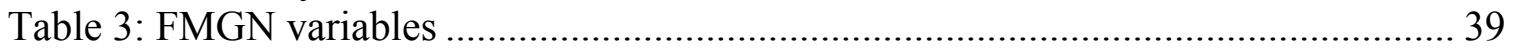

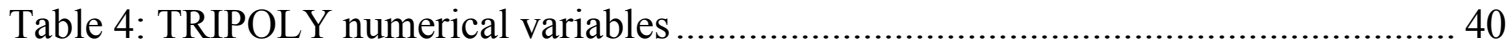

Table 5: Other variables used in TRIPOLY and modified from their default value ........ 40

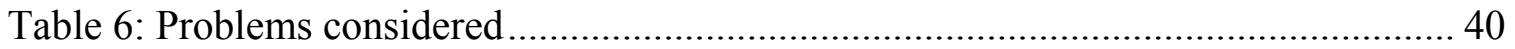

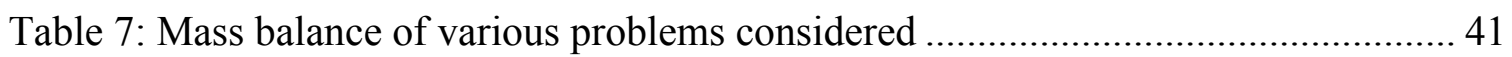

Table 8: BTC characteristics for porosity tests ....................................................... 41

Table 9: BTC characteristics for diffusion coefficient tests ......................................... 41

Table 10: BTC characteristics for retardation coefficient tests .................................... 42

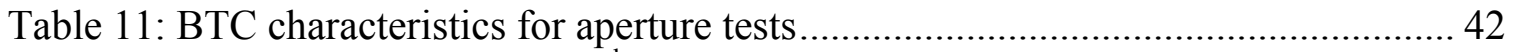

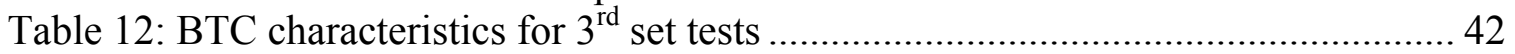

Table 13: Comparisons between the two cases of hydrofracking ................................. 43

Table 14: Summary of inferring physical parameters from withdrawal period of BTC (a check mark indicates a positive correlation) ........................................................... 43

Table 15: Summary of model studies in terms of their effect on the impact of the rock matrix (a check mark indicates a positive correlation) ............................................ 44 
Table 1: SWIW parameters

\begin{tabular}{|c|c|}
\hline Sequence & Parameters \\
\hline Injection & $t_{I}, q_{I}$ \\
\hline Chase fluid & $C=0, t_{C}, q_{I}=q_{C}$ \\
\hline Withdrawal & $t_{W}, q_{W}=-q_{I}$ \\
\hline
\end{tabular}

Table 2: Boundary conditions

\begin{tabular}{|c|c|c|c|c|}
\hline \multicolumn{2}{|c|}{ Boundary conditions } & Injection & Chase fluid period & Withdrawal \\
\hline \multirow{3}{*}{ Flow } & Outside & Dirichlet: $H=0$ & Dirichlet: $H=0$ & $\begin{array}{c}\text { Dirichlet: } \\
H=0\end{array}$ \\
\cline { 2 - 5 } & Well & Neumann: $q_{I}$ & Neumann: $q_{I}$ & Neumann: $-q_{I}$ \\
\hline \multirow{2}{*}{$\begin{array}{c}\text { Solute } \\
\text { transport }\end{array}$} & Outside & $\mathbf{X}$ & $\mathbf{X}$ & $\mathbf{X}$ \\
\cline { 2 - 5 } & Well & Neumann: $q_{I}$ & $\mathbf{X}$ & $\mathbf{X}$ \\
\hline
\end{tabular}

NB: $\mathbf{X}$ indicates that the node is treated as an internal node, so no boundary condition is necessary in this case

Table 3: FMGN variables

\begin{tabular}{|c|c|c|}
\hline Variable & Value & Significance \\
\hline APER & $10^{-4}$ & Fracture aperture (in meter) \\
\hline$B C O D E$ & 1 & Boundary code for each side of the flow region \\
\hline BVALUE & 0 & Value of the constant hydraulic head (in meter) \\
\hline LENGTH & 1 & Fracture length (in meter) \\
\hline NFRAC & 700 & Number of fractures per set \\
\hline NSETS & 2 & Number of fracture sets \\
\hline ORIEN & $5 / 95$ & $\begin{array}{l}\text { Orientation between the fracture axis and } \mathrm{x} \text { direction for } \\
\text { set } 1 \text { and set } 2 \text { respectively (in }{ }^{\circ} \text { ) }\end{array}$ \\
\hline XGENE, YGENE & 10 & $\begin{array}{l}\text { Dimension of the generated region in } \mathrm{x} \text { and } \mathrm{y} \text { direction } \\
\text { respectively (in meter) }\end{array}$ \\
\hline XMESH, YMESH & 10 & $\begin{array}{l}\text { Dimension of the flow region in } \mathrm{x} \text { and } \mathrm{y} \text { direction } \\
\text { respectively (in meter) }\end{array}$ \\
\hline
\end{tabular}


Table 4: TRIPOLY numerical variables

\begin{tabular}{|c|c|c|c|}
\hline \multirow[b]{2}{*}{ Variable } & \multicolumn{2}{|c|}{ Value } & \multirow[b]{2}{*}{ Significance } \\
\hline & Base Case & $\begin{array}{c}\text { Medium and } \\
\text { Small Problems }\end{array}$ & \\
\hline DCOFF & $10^{-2}$ & $10^{-4}$ & Used for removing nodes \\
\hline DCDIF & $10^{-1}$ & $10^{-1}$ & \multirow{3}{*}{ Used for inserting nodes } \\
\hline$D C O N$ & $10^{-2}$ & $10^{-3}$ & \\
\hline DEPS & $10^{-3}$ & $10^{-4}$ & \\
\hline LOGDIF & -1 & 2 & $\begin{array}{l}\text { Used to determine the criterion for inserting } \\
\text { and removing dispersive nodes }\end{array}$ \\
\hline TOLE & $10^{-2}$ & $10^{-5}$ & $\begin{array}{c}\text { Tolerance for numerical round-off: if } \\
\text { distance between } 2 \text { nodes is less than TOLE, } \\
\text { they are merged to } 1 \text { node }\end{array}$ \\
\hline
\end{tabular}

Table 5: Other variables used in TRIPOLY and modified from their default value

\begin{tabular}{|c|c|c|}
\hline Variable & Value & Significance \\
\hline DIFMOL & $2 \cdot 10^{-7}$ & $D_{f w}:$ molecular diffusion coefficient in fractures $\left(\mathrm{in}^{2} \cdot \mathrm{s}^{-1}\right)$ \\
\hline$I D O P O$ & 1 & Porous matrix is considered in simulation \\
\hline$I M O D E$ & 2 & Advection-dispersion in steady-state flow field \\
\hline$N P N$ & Node \# & Head value at the designed node (typically the node \# of the well) \\
\hline$P R R$ & Variable & Factor for increasing the time step $(\geq 1$, typically $1<P R R<1.1)$ \\
\hline$T M A X$ & Variable & Maximum simulation time allowed (in $\mathrm{s})$ \\
\hline
\end{tabular}

Table 6: Problems considered

\begin{tabular}{|c|c|c|c|}
\hline & Large Problem & Medium Problem & Small Problem \\
\hline \multirow{3}{*}{ Injection } & $\begin{array}{c}t_{I}=3600 \mathrm{~s} \\
(1 \mathrm{~h} \text { duration })\end{array}$ & $\begin{array}{c}t_{I}=3600 \mathrm{~s} \\
(1 \mathrm{~h} \text { duration })\end{array}$ & $\begin{array}{c}t_{I}=1800 \mathrm{~s} \\
(30 \text { min duration })\end{array}$ \\
& $q_{I}=2.8 \cdot 10^{-6} \mathrm{~m}^{3} \cdot \mathrm{s}^{-1}$ & $q_{I}=2.8 \cdot 10^{-6} \mathrm{~m}^{3} \cdot \mathrm{s}^{-1}$ & $q_{I}=2.8 \cdot 10^{-6} \mathrm{~m}^{3} \cdot \mathrm{s}^{-1}$ \\
\hline \multirow{2}{*}{ Chase fluid period } & $t_{C}=1.80 \cdot 10^{4} \mathrm{~s}$ & $\begin{array}{c}t_{C}=3600 \mathrm{~s} \\
(1 \mathrm{~h} \text { duration })\end{array}$ & $\begin{array}{c}t_{C}=2700 \mathrm{~s} \\
(45 \text { min duration })\end{array}$ \\
\hline \multirow{2}{*}{ Withdrawal } & $t_{W}=1.80 \cdot 10^{6} \mathrm{~s}$ & $t_{W}=1.80 \cdot 10^{6} \mathrm{~s}$ & $t_{W}=1.26 \cdot 10^{6} \mathrm{~s}$ \\
$(500 \mathrm{~h}$ duration $)$ & $(500 \mathrm{~h}$ duration $)$ & $(350 \mathrm{~h}$ duration $)$ \\
\hline
\end{tabular}


Table 7: Mass balance of various problems considered

\begin{tabular}{|c|c|c|c|}
\hline & $\begin{array}{c}\text { Large } \\
\text { Problem }\end{array}$ & Medium Problem & Small Problem \\
\hline Theoretical Injected Mass (in g) & 10.08 & 10.08 & 5.04 \\
\hline $\begin{array}{c}\text { Value of Injected Mass calculated } \\
\text { by the program (in g) }\end{array}$ & 12.62 & 10.12 & 5.23 \\
\hline Retrieved Mass (in g) & 13.98 & 8.978 & 4.316 \\
\hline Remaining Mass (in g) & 0.360 & 0.261 & 0.180 \\
\hline $\begin{array}{c}\text { Loss at the sides at the end of } \\
\text { the chase fluid period (in g) }\end{array}$ & 0.402 & $7.7410^{-7}$ & $2.3710^{-8}$ \\
\hline Recovery Ratio $^{\text {1 (in \%) }}$ & $\mathbf{1 3 9}$ & $\mathbf{8 9 . 1}$ & $\mathbf{8 5 . 6}$ \\
\hline Mass Balance (in \%) $^{\text {(in }}$ & $\mathbf{1 1 7}$ & $\mathbf{9 1 . 3}$ & $\mathbf{8 6 . 0}$ \\
\hline
\end{tabular}

${ }^{1}$ : as defined by Equation 14: Retrieved Mass / Theoretical Injected Mass

2: as defined by Equation 15: (Retrieved Mass + Remaining Mass + Loss out sides) /

Program Injected Mass

Table 8: BTC characteristics for porosity tests

\begin{tabular}{|c|c|c|c|c|c|}
\hline & $\mathbf{2 \%}$ & $\mathbf{6} \%$ & $\mathbf{1 0} \%$ & $\mathbf{1 5 \%}$ & $\mathbf{2 0} \%$ \\
\hline Retrieved Mass (in g) & 9.093 & 8.930 & $\mathbf{8 . 9 7 8}$ & 9.232 & 8.478 \\
\hline Remaining Mass (in g) & 0.104 & 0.194 & $\mathbf{0 . 2 6 1}$ & 0.350 & 0.378 \\
\hline Recovery Ratio (in \%) & 90.2 & 88.6 & $\mathbf{8 9 . 1}$ & 91.6 & 84.1 \\
\hline $\begin{array}{c}\text { Peak height (relative } \\
\text { concentration) }\end{array}$ & 0.143 & 0.110 & $\mathbf{0 . 1 0 0}$ & 0.096 & 0.081 \\
\hline Peak time (s) & 9017 & 8121 & $\mathbf{7 9 7 3}$ & 7626 & 7780 \\
\hline Slope tail 1 & -1.53 & -1.20 & $\mathbf{- 1 . 0 9}$ & -1.03 & -1.01 \\
\hline Length tail 1 & Normal & Normal & Normal & Quite long & Quite long \\
\hline Slope tail 2 & -1.71 & -1.67 & $\mathbf{- 1 . 6 5}$ & -1.63 & -1.62 \\
\hline Length tail 2 & Very Long & Long & Normal & Normal & Normal \\
\hline
\end{tabular}

Table 9: BTC characteristics for diffusion coefficient tests

\begin{tabular}{|c|c|c|c|c|}
\hline & $\mathbf{4 1 0}^{-\mathbf{1 0}}$ & $\mathbf{2 \cdot 1 0}^{-9}$ & $\mathbf{4 \cdot 1 0}^{-9}$ & $\mathbf{1 1 0}^{-8}$ \\
\hline Retrieved Mass (in g) & 8.785 & $\mathbf{8 . 9 7 8}$ & 9.461 & 9.618 \\
\hline Remaining Mass (in g) & 0.403 & $\mathbf{0 . 2 6 1}$ & 0.212 & 0.135 \\
\hline Recovery Ratio (in \%) & 87.2 & $\mathbf{8 9 . 1}$ & 93.9 & 95.4 \\
\hline $\begin{array}{c}\text { Peak height (relative } \\
\text { concentration) }\end{array}$ & 0.116 & $\mathbf{0 . 1 0 0}$ & 0.099 & 0.088 \\
\hline Peak time (s) & 8348 & $\mathbf{7 9 7 3}$ & 7626 & 7632 \\
\hline Slope tail 1 & -1.25 & $\mathbf{- 1 . 0 9}$ & -1.00 & -0.94 \\
\hline Length tail 1 & Normal & Normal & Little short & Little short \\
\hline Slope tail 2 & -1.60 & $\mathbf{- 1 . 6 5}$ & -1.77 & -1.93 \\
\hline Length tail 2 & Normal & Normal & Little short & Little short \\
\hline
\end{tabular}


Table 10: BTC characteristics for retardation coefficient tests

\begin{tabular}{|c|c|c|c|c|c|}
\hline & $\boldsymbol{R}=\mathbf{1}$ & $\boldsymbol{R}=\mathbf{2}$ & $\boldsymbol{R}=\mathbf{5}$ & $\boldsymbol{R}=\mathbf{1 0}$ & $\boldsymbol{R}=\mathbf{2 5}$ \\
\hline Retrieved Mass (in g) & $\mathbf{8 . 9 7 8}$ & 9.032 & 7.469 & 8.551 & 5.929 \\
\hline Remaining Mass (in g) & $\mathbf{0 . 2 6 1}$ & 0.245 & 0.147 & 0.138 & 0.052 \\
\hline Recovery Ratio (in \%) & $\mathbf{8 9 . 1}$ & 89.6 & 74.1 & 84.8 & 58.8 \\
\hline $\begin{array}{c}\text { Peak height (relative } \\
\text { concentration) }\end{array}$ & $\mathbf{0 . 1 0 0}$ & 0.096 & 0.074 & 0.079 & 0.052 \\
\hline Peak time (s) & $\mathbf{7 9 7 3}$ & 7626 & 7783 & 7799 & 7644 \\
\hline Slope tail 1 & $\mathbf{- 1 . 0 9}$ & -1.06 & -1.06 & -1.03 & -1.03 \\
\hline Length tail 1 & Normal & Normal & Long & Long & Normal \\
\hline Slope tail 2 & $\mathbf{- 1 . 6 5}$ & -1.58 & -1.51 & -1.42 & -1.26 \\
\hline Length tail 2 & Normal & Normal & Quite short & Short & Normal \\
\hline
\end{tabular}

Table 11: BTC characteristics for aperture tests

\begin{tabular}{|c|c|c|c|c|}
\hline & Base case & x 2 & x 5 & x 10 \\
\hline Retrieved Mass (in g) & $\mathbf{8 . 9 7 8}$ & 8.833 & 9.315 & 10.003 \\
\hline Remaining Mass (in g) & $\mathbf{0 . 2 6 1}$ & 0.237 & 0.207 & 0.177 \\
\hline Recovery Ratio (in \%) & $\mathbf{8 9 . 1}$ & 87.6 & 92.4 & 99.2 \\
\hline $\begin{array}{c}\text { Peak height (relative } \\
\text { concentration) }\end{array}$ & $\mathbf{0 . 1 0 0 2}$ & 0.0998 & 0.1136 & 0.1341 \\
\hline Peak time (s) & $\mathbf{7 9 7 3}$ & 8258 & 8784 & 8784 \\
\hline Slope tail 1 & $\mathbf{- 1 . 0 9}$ & -1.11 & -1.25 & -1.39 \\
\hline Length tail 1 & Normal & Normal & Normal & Quite short \\
\hline Slope tail 2 & $\mathbf{- 1 . 6 5}$ & -1.69 & -1.68 & -1.70 \\
\hline Length tail 2 & Normal & Normal & Quite long & Quite long \\
\hline Pressure (m) & $\mathbf{0 . 8 0 0 6}$ & 0.1001 & 0.0064 & $8.006 \cdot 10^{-4}$ \\
\hline
\end{tabular}

Table 12: BTC characteristics for $3^{\text {rd }}$ set tests

\begin{tabular}{|c|c|c|c|c|}
\hline & Base case & $+\mathbf{2 0 0}$ & $\mathbf{+ 4 5 0}$ & $\mathbf{+ 7 0 0}$ \\
\hline Retrieved Mass (in g) & $\mathbf{8 . 9 7 8}$ & 9.337 & 9.686 & 9.699 \\
\hline Remaining Mass (in g) & $\mathbf{0 . 2 6 1}$ & 0.290 & 0.278 & $-0.121 \cdot 10^{3}$ \\
\hline Recovery Ratio (in \%) & $\mathbf{8 9 . 1}$ & 92.6 & 96.1 & 96.2 \\
\hline $\begin{array}{c}\text { Peak height (relative } \\
\text { concentration) }\end{array}$ & $\mathbf{0 . 1 0 0 2}$ & 0.1011 & 0.1018 & 0.1008 \\
\hline Peak time (s) & $\mathbf{7 9 7 3}$ & 8057 & 8057 & 7763 \\
\hline Slope tail 1 & $\mathbf{- 1 . 0 9}$ & -1.05 & -1.05 & -1.04 \\
\hline Length tail 1 & Normal & Quite long & Long & Long \\
\hline Slope tail 2 & $\mathbf{- 1 . 6 5}$ & -1.70 & -1.85 & -1.95 \\
\hline Length tail 2 & Normal & Quite short & Short & Short \\
\hline Pressure (m) & $\mathbf{0 . 8 0 0 6}$ & 0.7580 & 0.5689 & 0.4847 \\
\hline
\end{tabular}


Table 13: Comparisons between the two cases of hydrofracking

\begin{tabular}{|c|c|c|c|c|c|c|c|c|}
\hline $\begin{array}{l}\text { Aperture } \\
\text { (mm) }\end{array}$ & Ratio & $\begin{array}{l}\Delta \mathbf{P} \\
(\boldsymbol{m})\end{array}$ & $\begin{array}{c}\text { Peak } \\
\text { height }\end{array}$ & $\begin{array}{c}\text { Number } \\
\text { of } \\
\text { fractures }\end{array}$ & $\begin{array}{l}\text { Total length } \\
\text { of fractures } \\
(\mathrm{m})\end{array}$ & Ratio & $\begin{array}{l}\Delta \mathbf{P} \\
(\mathbf{m})\end{array}$ & $\begin{array}{c}\text { Peak } \\
\text { height }\end{array}$ \\
\hline 0.1 & 1 & 0.8006 & 0.1002 & Base case & 973 & 1 & 0.8006 & 0.1002 \\
\hline 0.125 & 1.25 & 0.4099 & 0.1019 & +200 & 1188 & 1.22 & 0.7580 & 0.1011 \\
\hline 0.15 & 1.5 & 0.2372 & 0.0973 & +450 & 1440 & 1.48 & 0.5689 & 0.1018 \\
\hline 0.175 & 1.75 & 0.1494 & 0.0988 & +700 & 1673 & 1.72 & 0.4847 & 0.1008 \\
\hline 0.2 & 2 & 0.1001 & 0.0999 & & & & & \\
\hline 0.5 & 5 & 0.0064 & 0.1136 & & & & & \\
\hline 1 & 10 & 0.0008 & 0.1341 & & & & & \\
\hline
\end{tabular}

Table 14: Summary of inferring physical parameters from withdrawal period of BTC (a check mark indicates a positive correlation)

\begin{tabular}{|c|c|c|c|c|c|c|c|c|}
\hline & \multicolumn{2}{|c|}{ Peak height } & \multicolumn{2}{c|}{-3/2 slope } & \multicolumn{2}{c|}{ Long tail } & \multicolumn{2}{c|}{ Short tail } \\
\hline & High & Low & Part 1 & Part 2 & Part 1 & Part 2 & Part 1 & Part 2 \\
\hline Low porosity & $\checkmark$ & & $\checkmark$ & & & $\checkmark$ & & \\
\hline High porosity & & $\checkmark$ & & $\checkmark$ & $\checkmark$ & & & $\checkmark$ \\
\hline Small diffusion & $\checkmark$ & & & $\checkmark$ & & & & \\
\hline Large diffusion & & $\checkmark$ & & & & & $\checkmark$ & $\checkmark$ \\
\hline Retardation & & $\checkmark$ & & $\checkmark$ & $\checkmark$ & & & $\checkmark$ \\
\hline Big aperture & $\checkmark$ & & $\checkmark$ & & & $\checkmark$ & $\checkmark$ & \\
\hline More fractures & & & & & $\checkmark$ & & & $\checkmark$ \\
\hline
\end{tabular}


Table 15: Summary of model studies in terms of their effect on the impact of the rock matrix (a check mark indicates a positive correlation)

\begin{tabular}{|c|c|c|c|c|c|c|}
\hline \multicolumn{7}{|c|}{ Strong matrix impact } \\
\hline \multicolumn{7}{|c|}{ High porosity - Large diffusion $-R>1-$ Small aperture - More fractures } \\
\hline \multirow{7}{*}{$\begin{array}{l}\text { Processes \& } \\
\text { Spatial } \\
\text { distribution }\end{array}$} & 20 & High $\Phi^{M}$ & High $D_{e}$ & $R>1$ & Small aperture & $3^{\text {rd }}$ set \\
\hline & Small extent of plume & $\checkmark$ & $\checkmark$ & $\checkmark$ & & $\begin{array}{c}\checkmark \\
\text { (slightly) }\end{array}$ \\
\hline & Few blocks exposed & $\checkmark$ & $\checkmark$ & $\checkmark$ & & $\checkmark$ \\
\hline & $\begin{array}{l}\text { Lots of diffusion into } \\
\text { each matrix block }\end{array}$ & $\checkmark$ & $\checkmark$ & $\checkmark$ & $\checkmark$ & \\
\hline & $\begin{array}{l}\text { High capacity, may not } \\
\text { see saturation }\end{array}$ & $\checkmark$ & & $\checkmark$ & & \\
\hline & $\begin{array}{l}\text { Same capacity as base } \\
\text { case, more likely to see } \\
\text { saturation }\end{array}$ & & $\checkmark$ & & $\checkmark$ & \\
\hline & $\begin{array}{l}\text { More small blocks, } \\
\text { more saturation }\end{array}$ & & & & & $\checkmark$ \\
\hline \multirow{4}{*}{ BTC } & Low peak & $\checkmark$ & $\checkmark$ & & & \\
\hline & Early peak & $\checkmark$ & $\checkmark$ & & & \\
\hline & Two linear trends in tail & $\checkmark$ & & $\checkmark$ & $\checkmark$ & $\checkmark$ \\
\hline & $\begin{array}{l}\text { Late time behavior close } \\
\text { to }-3 / 2 \log \text { slope }\end{array}$ & $\checkmark$ & & & & \\
\hline
\end{tabular}

\section{Weak matrix impact}

\begin{tabular}{|c|l|c|c|c|c|}
\hline \multirow{5}{*}{\begin{tabular}{c} 
Processes \& $\begin{array}{c}\text { Spatial } \\
\text { distribution }\end{array}$ \\
\cline { 2 - 6 }
\end{tabular}} & Low $\boldsymbol{\Phi}^{M}$ & Low $\boldsymbol{D}_{\boldsymbol{e}}$ & $\boldsymbol{R}=\mathbf{1}$ & Big aperture \\
\hline & Lots of blocks exposed & $\checkmark$ & $\checkmark$ & $\checkmark$ & \\
\cline { 2 - 6 } & $\begin{array}{l}\text { Low diffusion into each } \\
\text { matrix block }\end{array}$ & $\checkmark$ & $\checkmark$ & $\checkmark$ & $\checkmark$ \\
& $\begin{array}{l}\text { Little capacity, possibly } \\
\text { more saturation }\end{array}$ & $\checkmark$ & & $\checkmark$ & \\
\cline { 2 - 6 } & $\begin{array}{l}\text { Same capacity as base } \\
\text { case, less likely to have } \\
\text { saturation }\end{array}$ & & $\checkmark$ & & $\checkmark$ \\
\hline \multirow{2}{*}{ BTC } & High peak & & $\checkmark$ & & \\
\hline & Late peak & $\checkmark$ & $\checkmark$ & $\checkmark$ & $\checkmark$ \\
\hline & Single linear trend in tail & $\checkmark$ & & & $\checkmark$ \\
\hline
\end{tabular}




\section{List of Figures}

Figure 1: SWIW test principle

Figure 2: Flow chart for use of programs ................................................................ 47

Figure 3: 1D concentration profile for diffusive transport in matrix blocks..................... 48

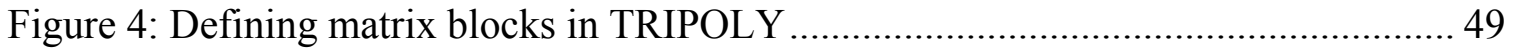

Figure 5: Geometry generated by FMGN .................................................................. 50

Figure 6: Calculation of the polygons defining the block geometry made by POLY ...... 51

Figure 7: Sample of "bad geometry" generated by FMGN ………………………......... 52

Figure 8: Matrix block size (in $\mathrm{m}$ ) distribution for the selected geometry ...................... 53

Figure 9: Tracer breakthrough curve (BTC) ……………......................................... 54

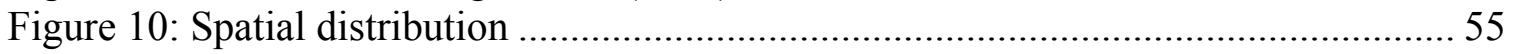

Figure 11: Breakthrough curve for large problem ...................................................... 56

Figure 12: Breakthrough curve for medium problem ................................................... 57

Figure 13: Breakthrough curve for small problem ..................................................... 58

Figure 14: Breakthrough curve with modeled tails....................................................... 59

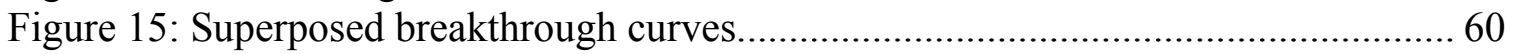

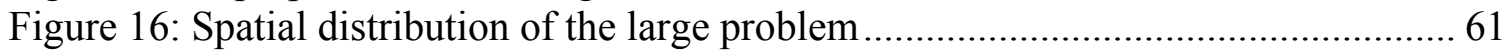

Figure 17: Spatial distribution of the medium problem.................................................. 62

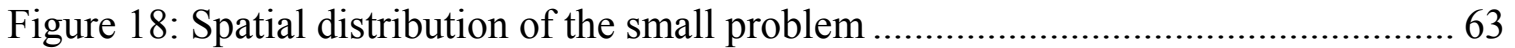

Figure 19: BTCs for porosity (top - log-log scales; bottom - linear-linear scales) ......... 64

Figure 20: Evolution of spatial distribution for different porosity values ........................ 66

Figure 21: BTCs for diffusion coefficients (top - log-log scales; bottom - linear-linear

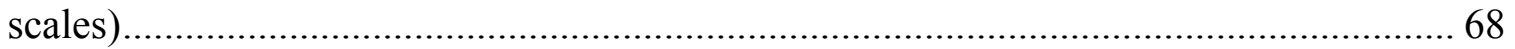

Figure 22: Evolution of spatial distribution for diffusion coefficients ............................ 70

Figure 23: BTCs for retardation coefficients (top - log-log scales; bottom - linear-linear

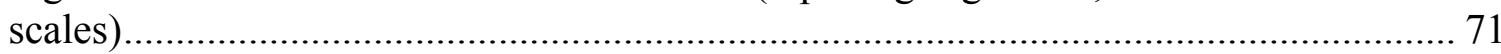

Figure 24: Evolution of spatial distribution for retardation coefficients ......................... 74

Figure 25: BTCs for apertures (top - log-log scales; bottom - linear-linear scales) ........ 74

Figure 26: Evolution of spatial distribution for different apertures ................................. 78

Figure 27: Histograms of the new block size (m) distribution when adding a third set... 79

Figure 28: BTCs for $3^{\text {rd }}$ sets (top - log-log scales; bottom - linear-linear scales) ........... 81

Figure 29: Evolution of spatial distribution for adding a $3^{\text {rd }}$ set ..................................... 83

Figure 30: BTC for small aperture enlargements ........................................................ 84

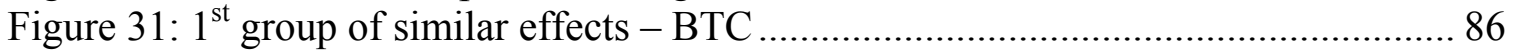

Figure 32: $1^{\text {st }}$ group of similar effects - Spatial Distribution ........................................... 87

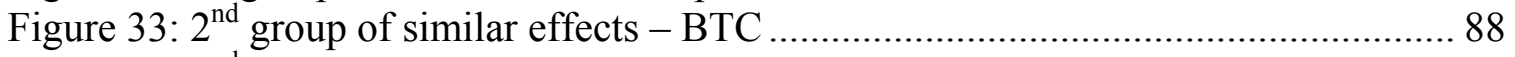

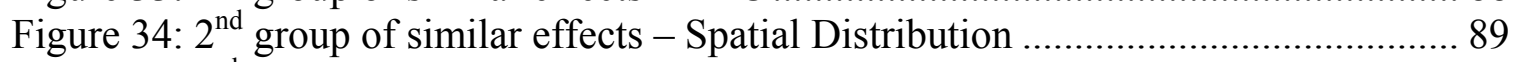

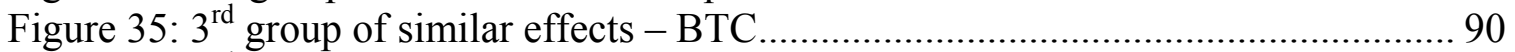

Figure 36: $3^{\text {rd }}$ group of similar effects - Spatial Distribution............................................ 91 
Figure 1: SWIW test principle

Schematic diagram of particle travel path during a two-well test (a) and a SWIW test (b). Arrows represent advection through the fracture and circles and ovals represent finite blocks into which diffusion and sorption may occur. I and W stand for Injection well and Withdrawal well, respectively. In SWIW test, matrix blocks are traversed by tracers twice (outwards and return) but in two-well test, matrix blocks are traversed only once with new blocks seen at all time steps. So in SWIW test, matrix blocks are more likely to be saturated by tracers, but in two-well test, they are not saturated.

(a)

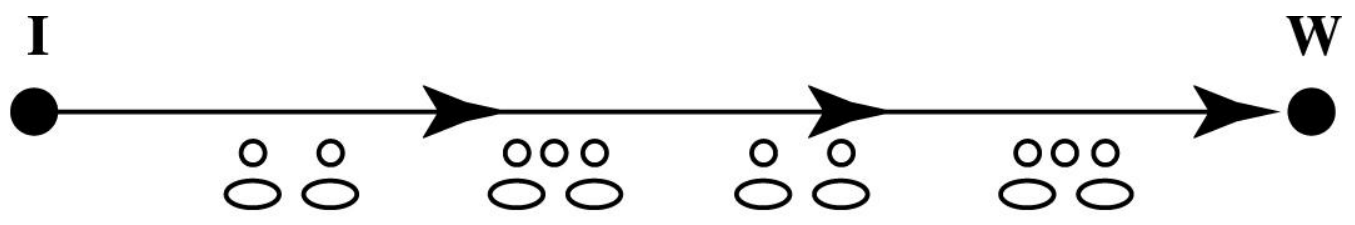

(b)

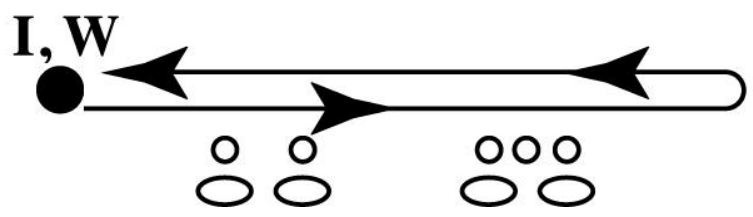

46 
Figure 2: Flow chart for use of programs

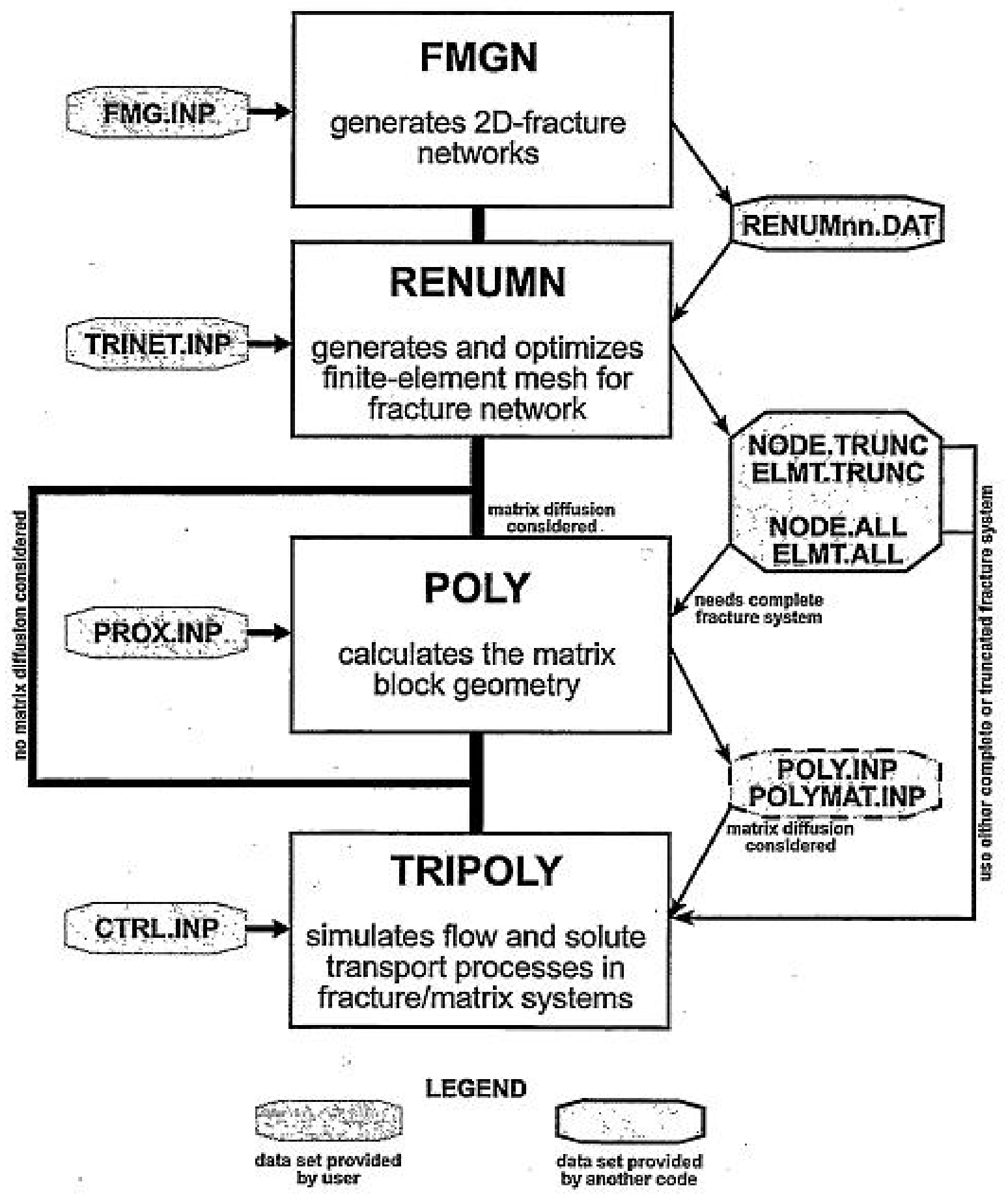


Figure 3: 1D concentration profile for diffusive transport in matrix blocks

$s$ is a local coordinate perpendicular to the adjacent fractures. This local coordinate is equal to zero at the fracture-matrix interface, and it has an upper limit at $s=S$ which is the maximum orthogonal distance of any location inside the block to the nearest fracture. $A(s)$ is the interface area for transport in the matrix blocks at a certain distance $s$ from the surface. Hence, for $s=0$, this area is equal to the contact area with the fracture, i.e. equal to the surface area $A_{0}$ of the matrix blocks, and for blocks of limited extent, it steadily decreases when approaching the block center $(s=S)$.
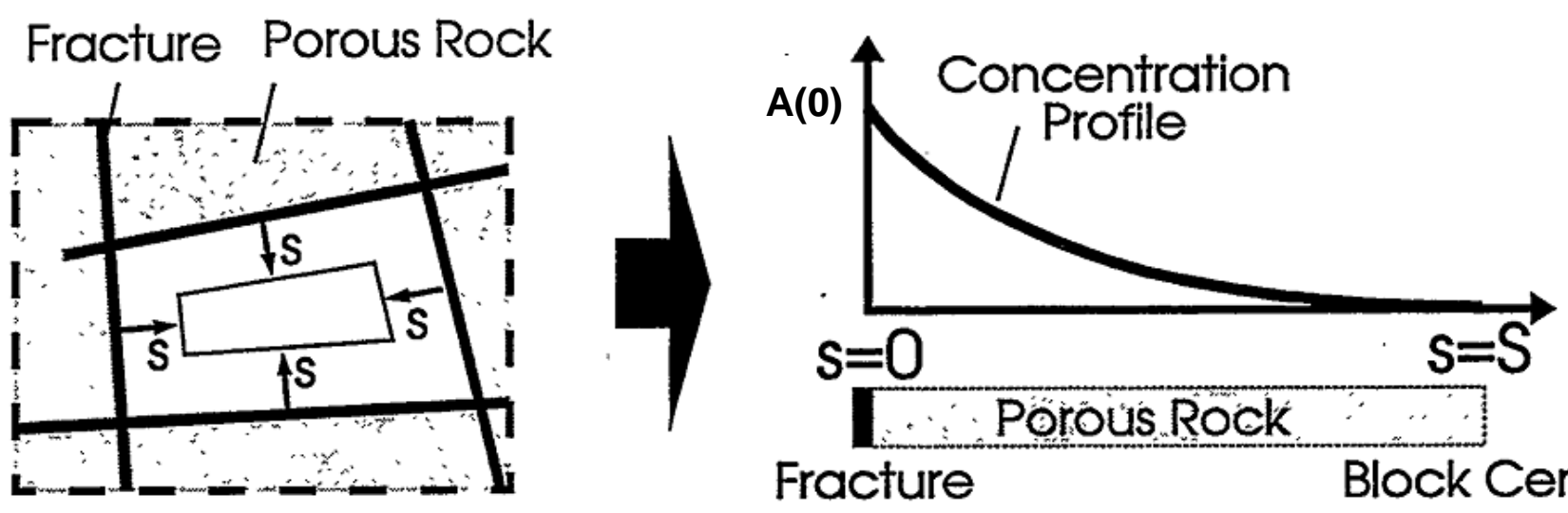

Fracture 
Figure 4: Defining matrix blocks in TRIPOLY

Each node of the fracture network is connected to a certain number of blocks (polygons), i.e., one polygon for dead-end nodes (e.g. node 12), two polygons for moving nodes between fractures intersections (e.g. nodes 33 and 78), and more than two polygons for fixed nodes located on fracture intersections (e.g. nodes 34, 48, 67, and 109). Each of those node-polygon connections is thereby related to a one-dimensional concentration distribution in the matrix.

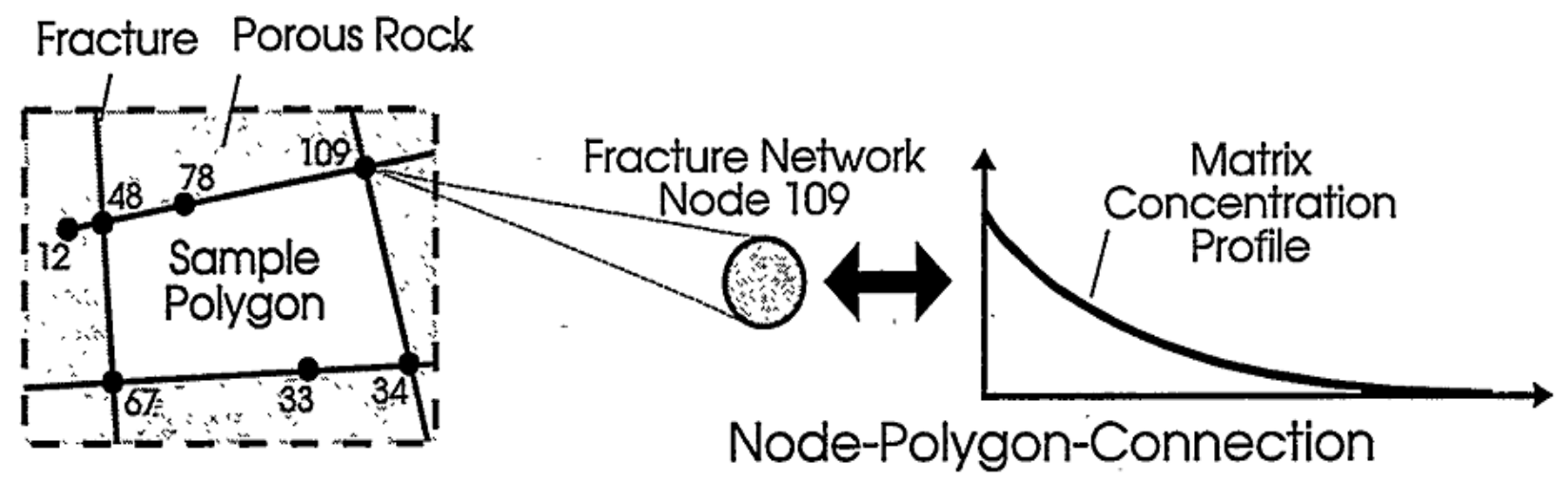

\section{Matrix Input Parameters in TRIPOLY:}

material properties: geometry: e.g. porosity, molecular diffusion coefficient

surface polygon (here: $67 ; 33 ; 34 ; 109 ; 78 ; 48$ )

interface function, maximum orthogonal distance $S$ 
Figure 5: Geometry generated by FMGN

The circle indicates the position of the well node.

\section{Geometry generated by FMGN}

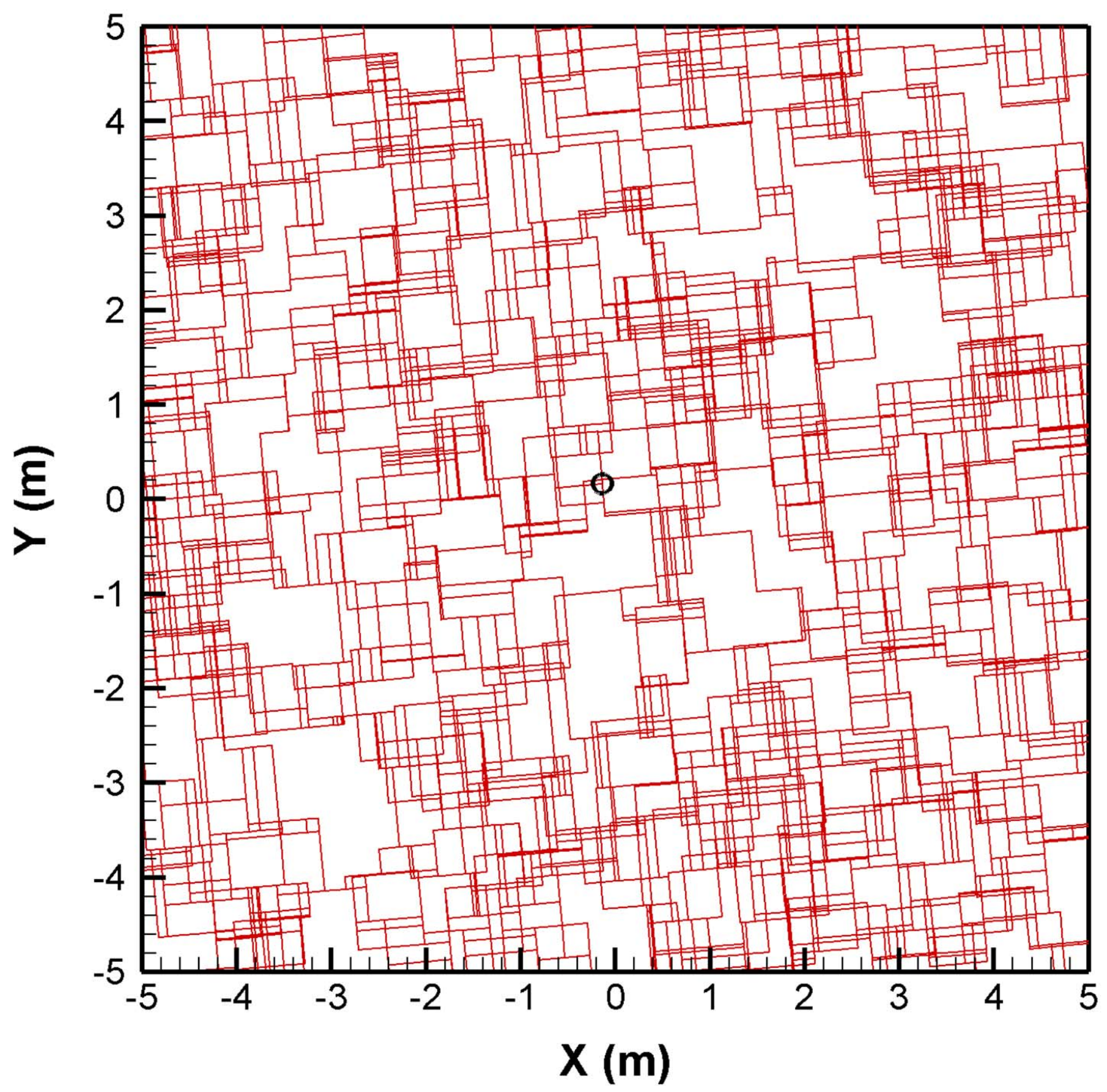


Figure 6: Calculation of the polygons defining the block geometry made by POLY

\section{Step 1:}

- extend dead-end fractures

- define convex polygons
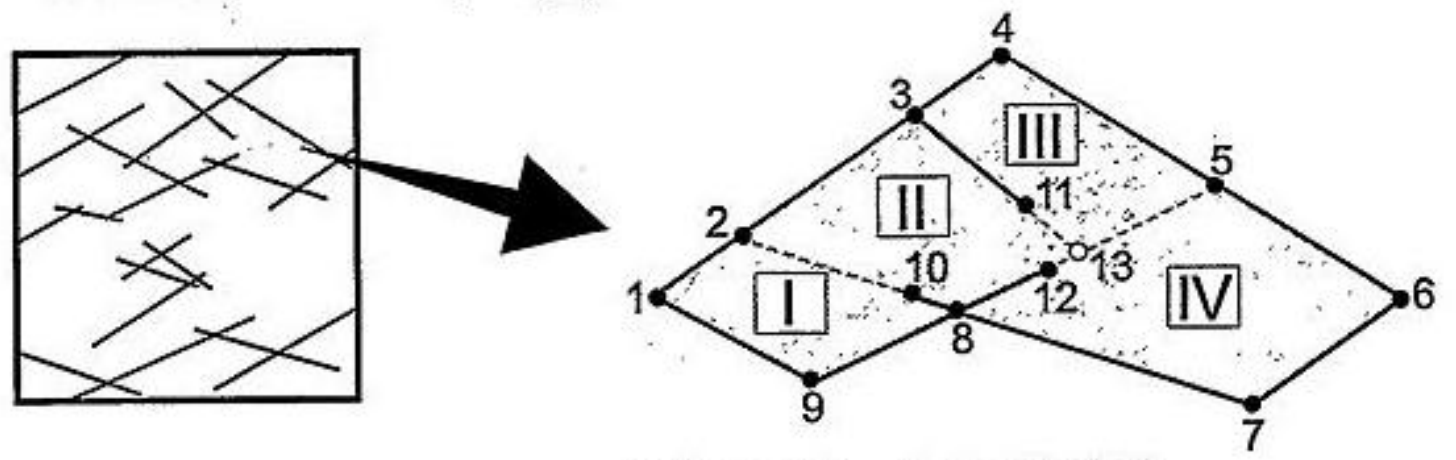

polygon I: $1,2,10,8,9$

polygon II: $2,3,11,13,12,8,10$

polygon III: $3,4,5,13,11$

polygon IV: $5,6,7,8,12,13$

\section{Step 2:}

- connect convex polygons

- create complicated polygon

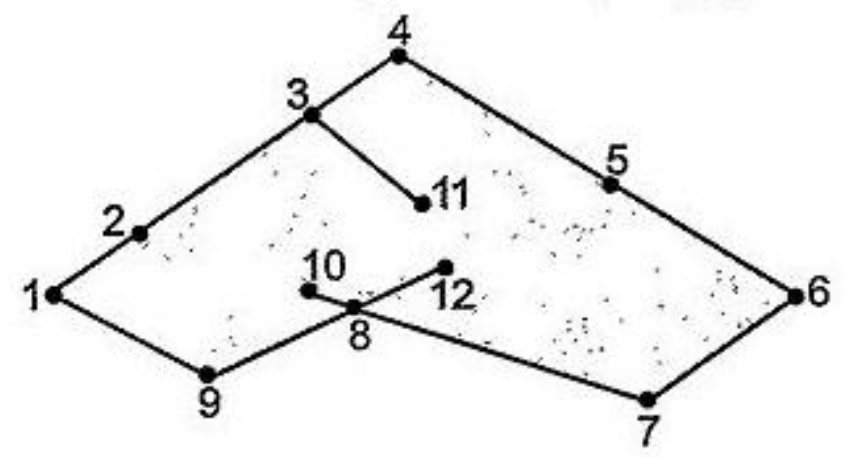

polygon with dead-ends: $\quad 1,2,3,11,3,4,5,6,7,8,12,8,10,8,9$ polygon without dead-ends: $1,2,3,4,5,6,7,8,9$ 
Figure 7: Sample of "bad geometry" generated by FMGN

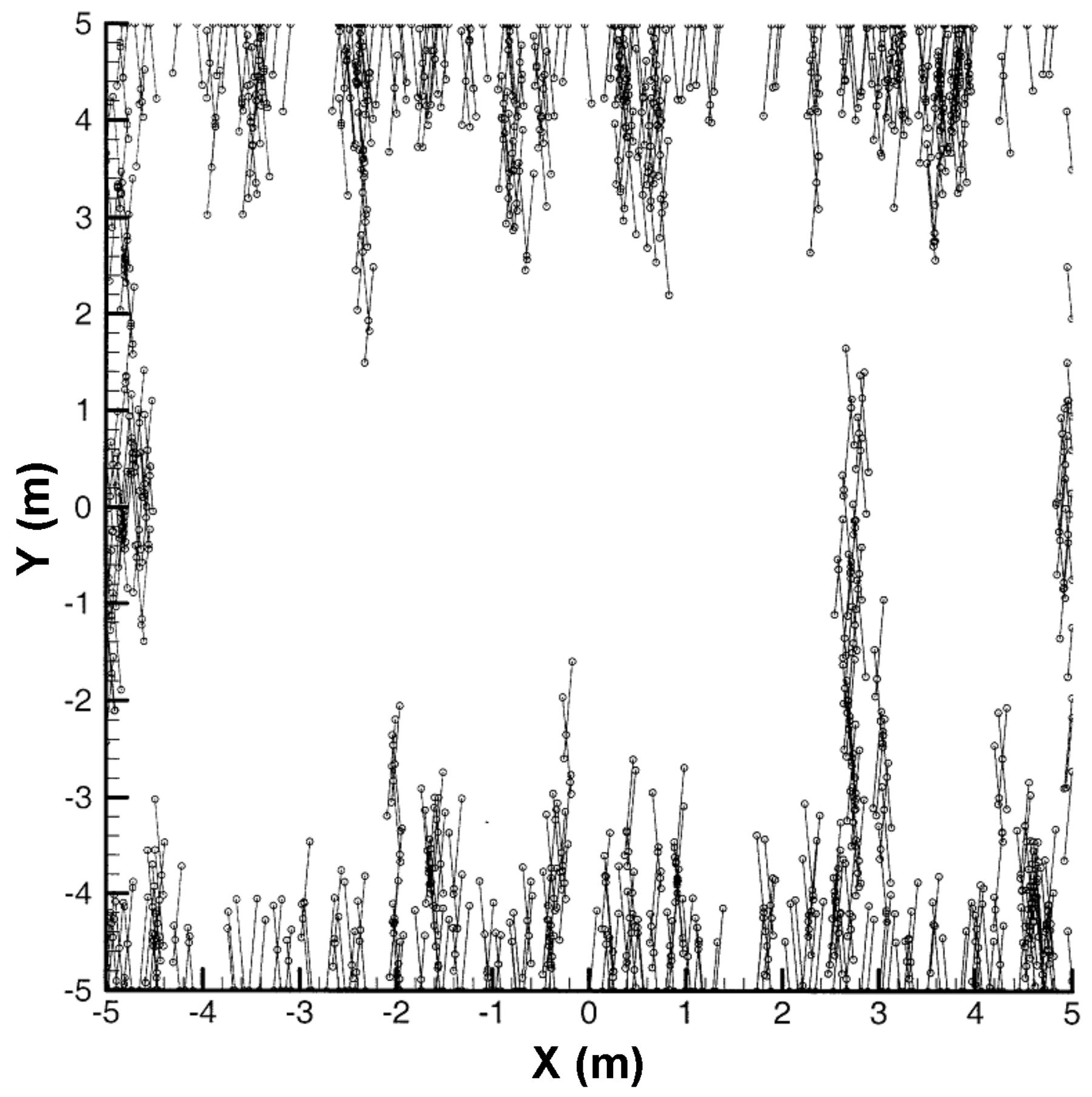


Figure 8: Matrix block size (in $\mathrm{m}$ ) distribution for the selected geometry

$S$ (expressed in meters) is the value of the maximum orthogonal distance of points in the matrix blocks to the adjacent fractures.

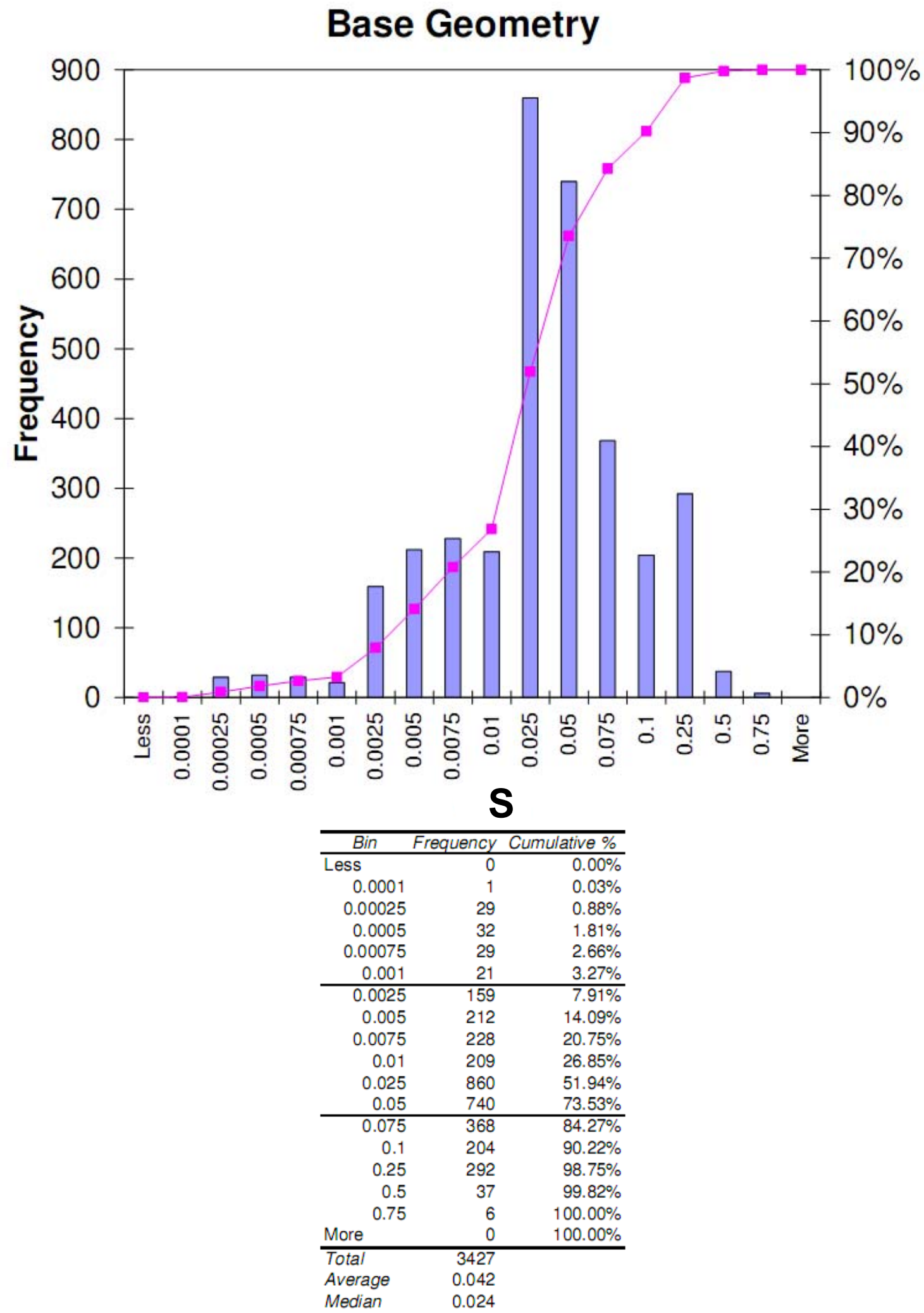


Figure 9: Tracer breakthrough curve (BTC)

\section{Sample of a BTC}

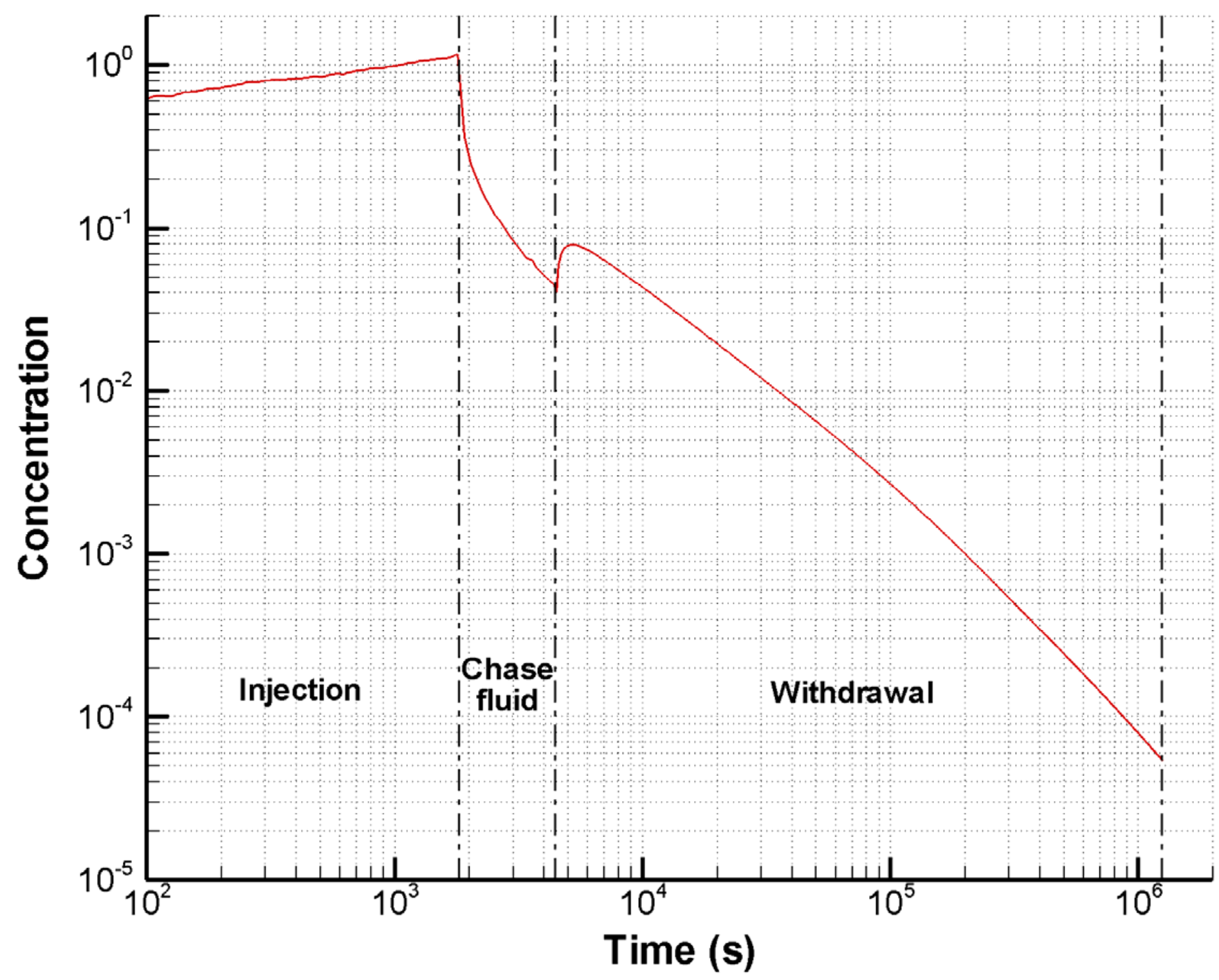


Figure 10: Spatial distribution

\section{Sample of a spatial distribution}
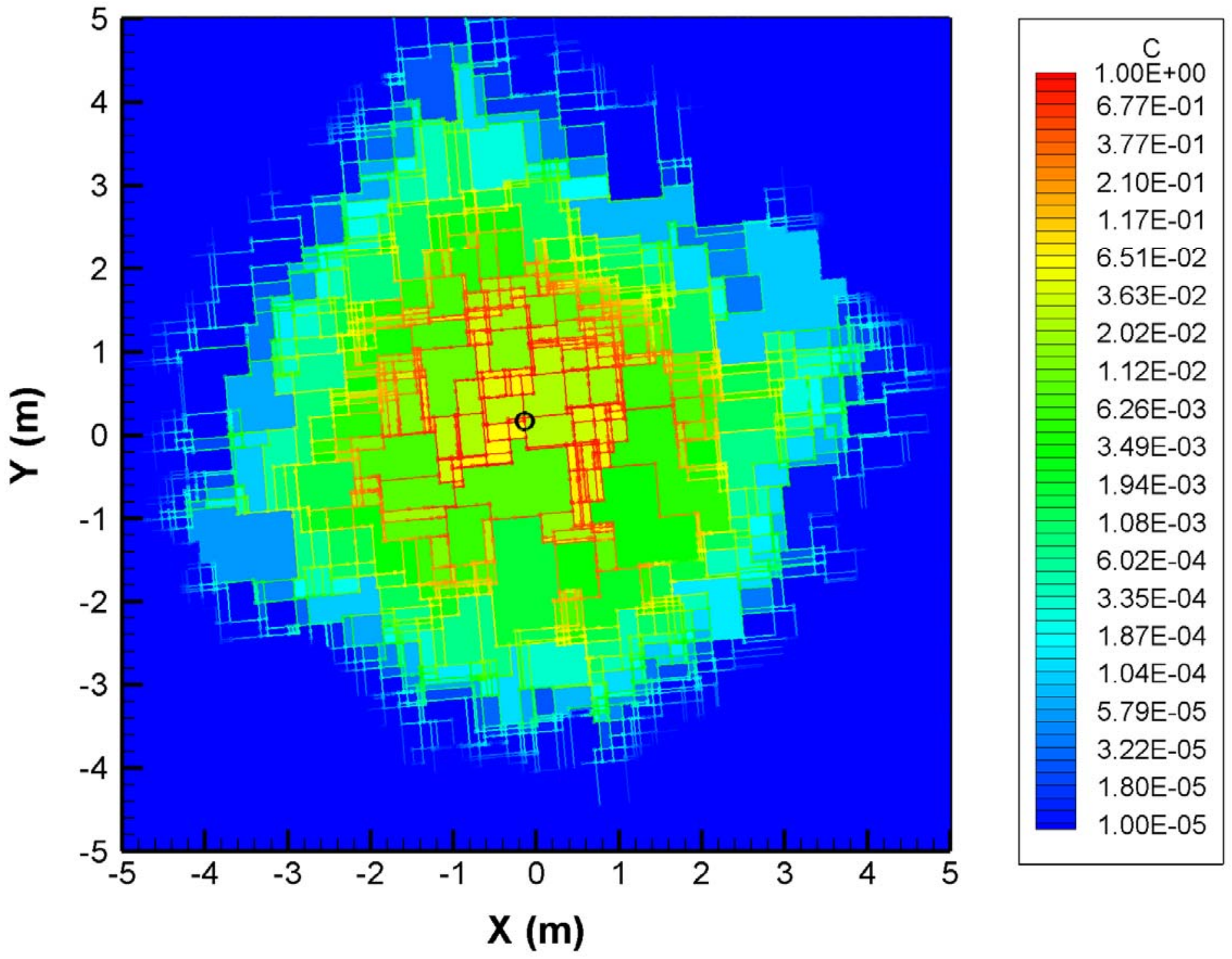
Figure 11: Breakthrough curve for large problem

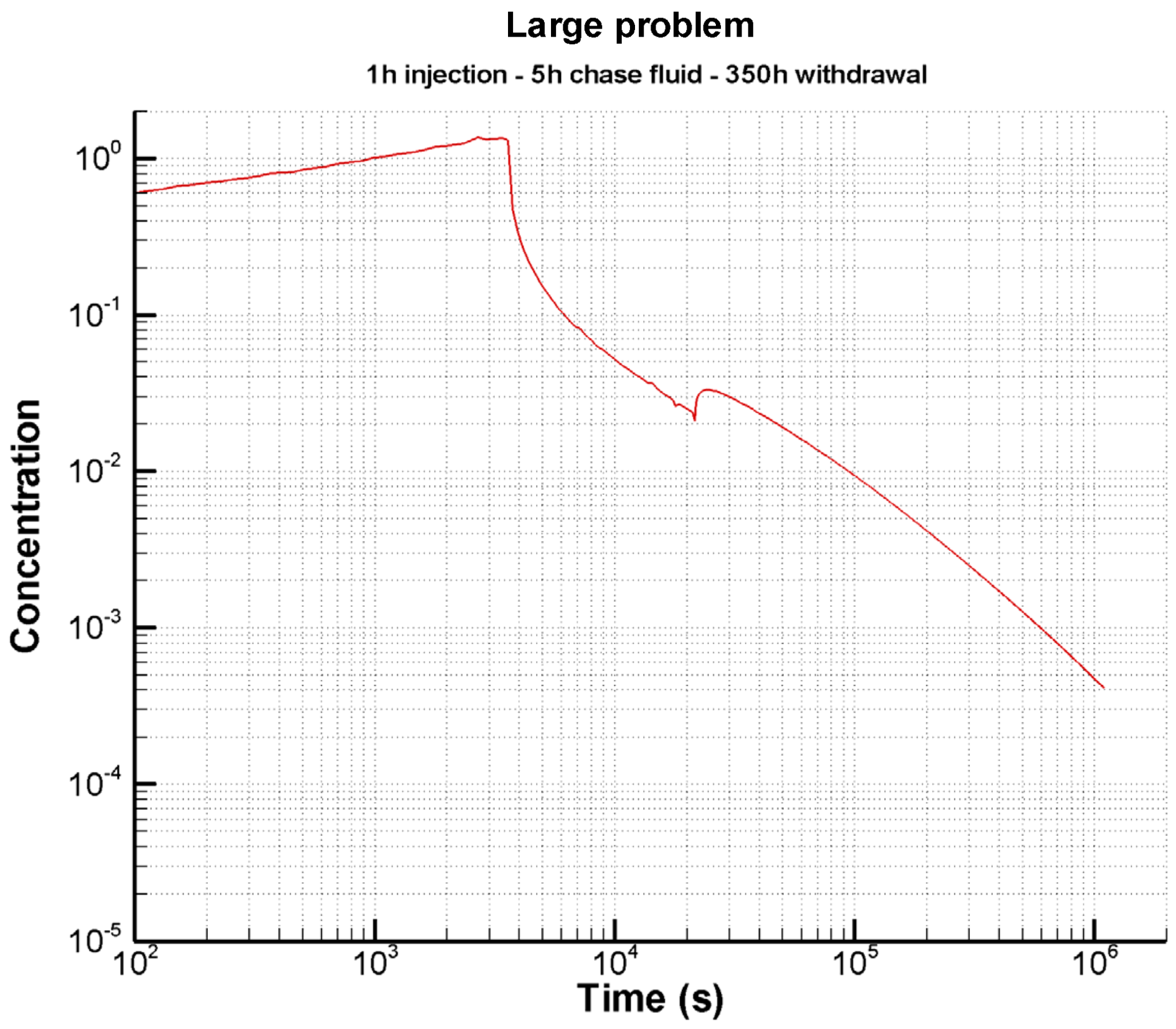


Figure 12: Breakthrough curve for medium problem

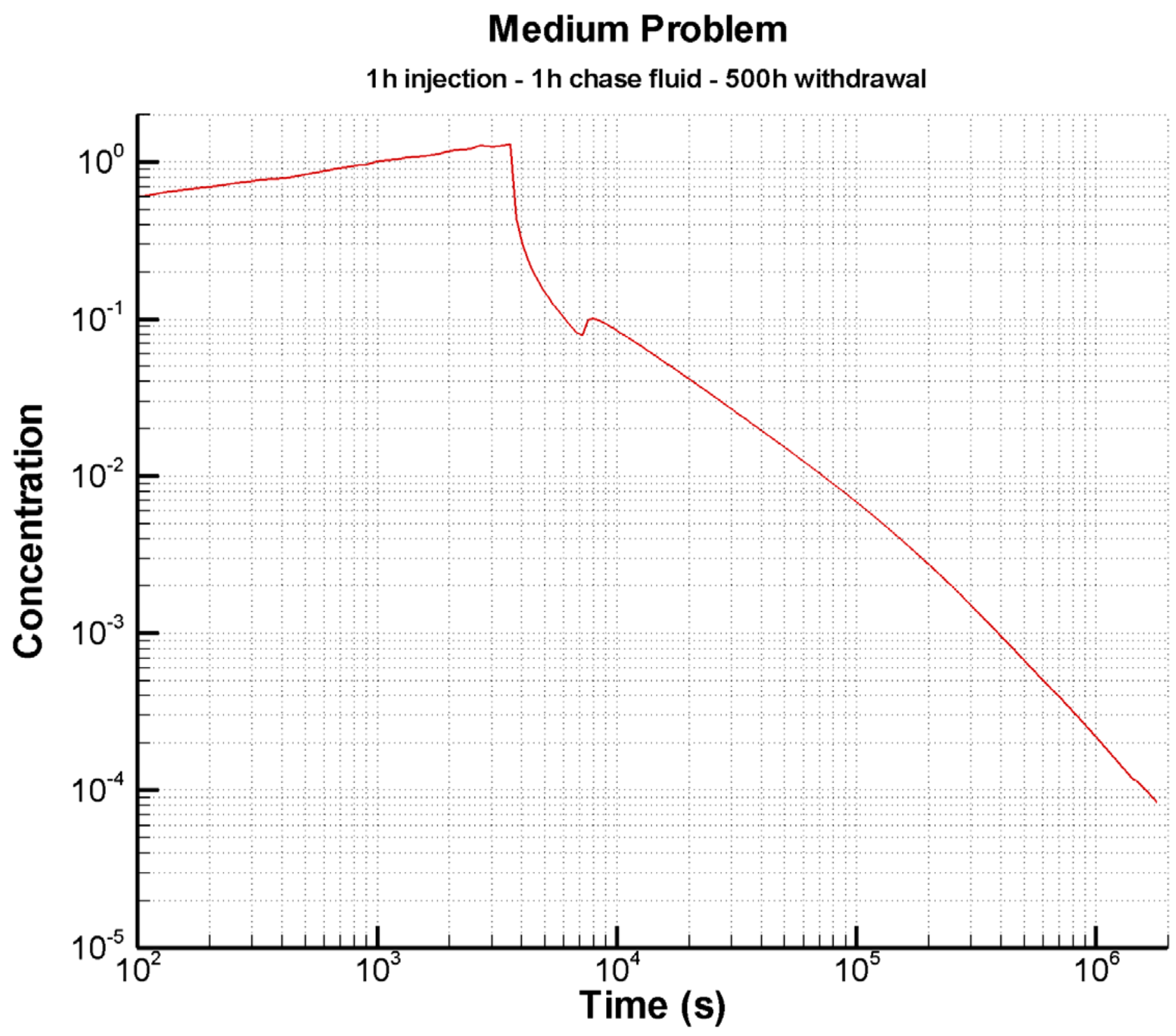


Figure 13: Breakthrough curve for small problem

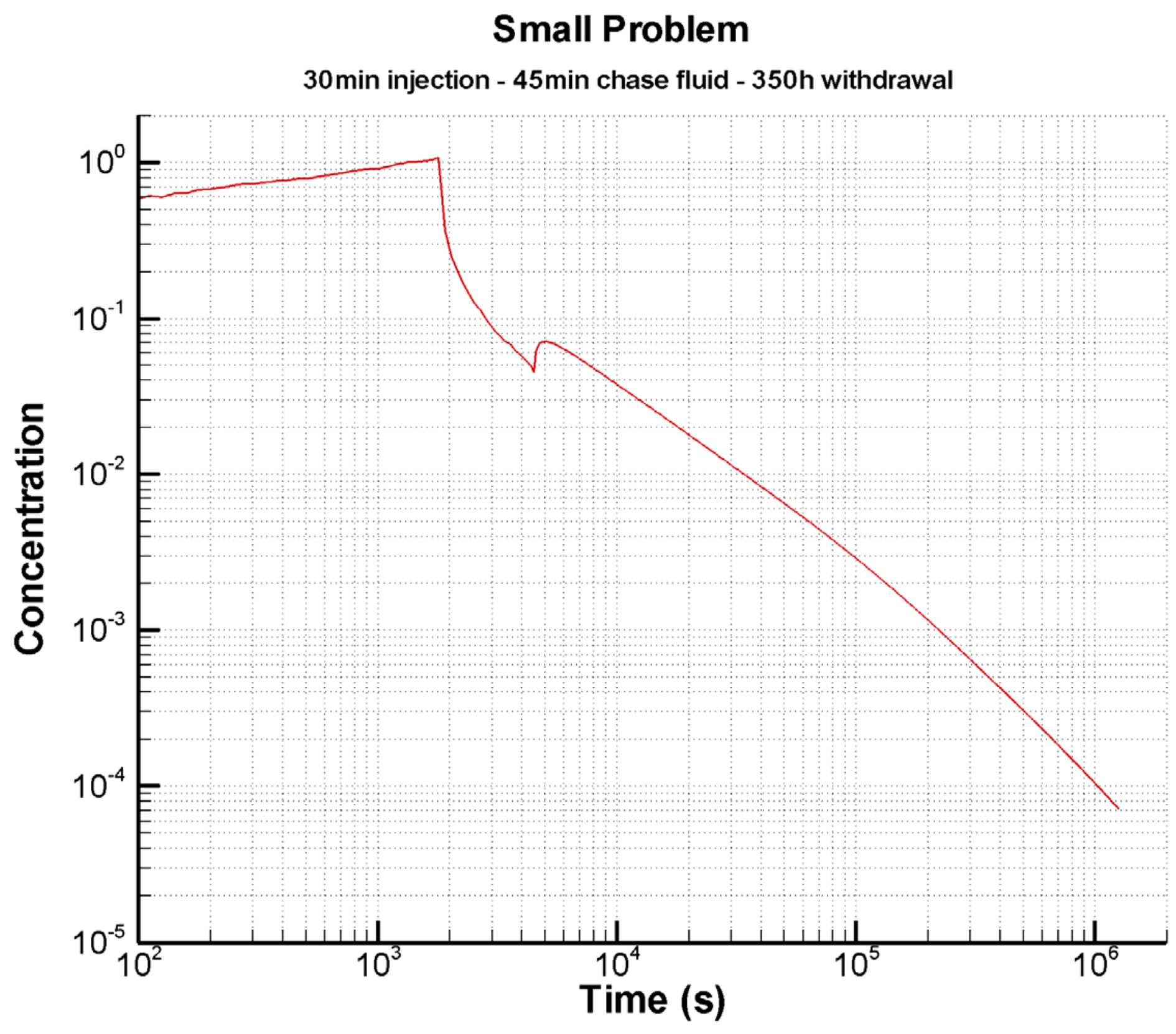


Figure 14: Breakthrough curve with modeled tails

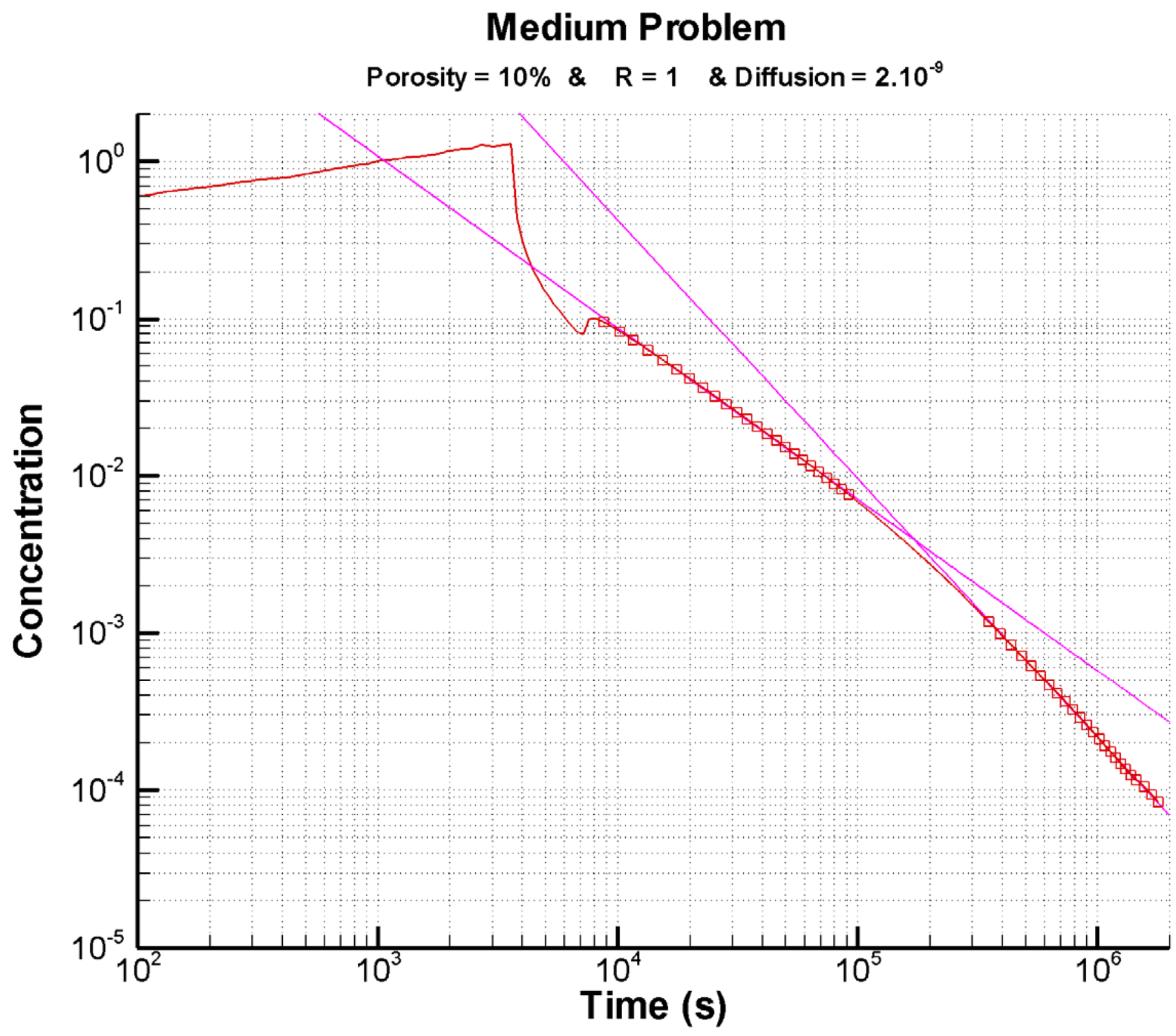


Figure 15: Superposed breakthrough curves

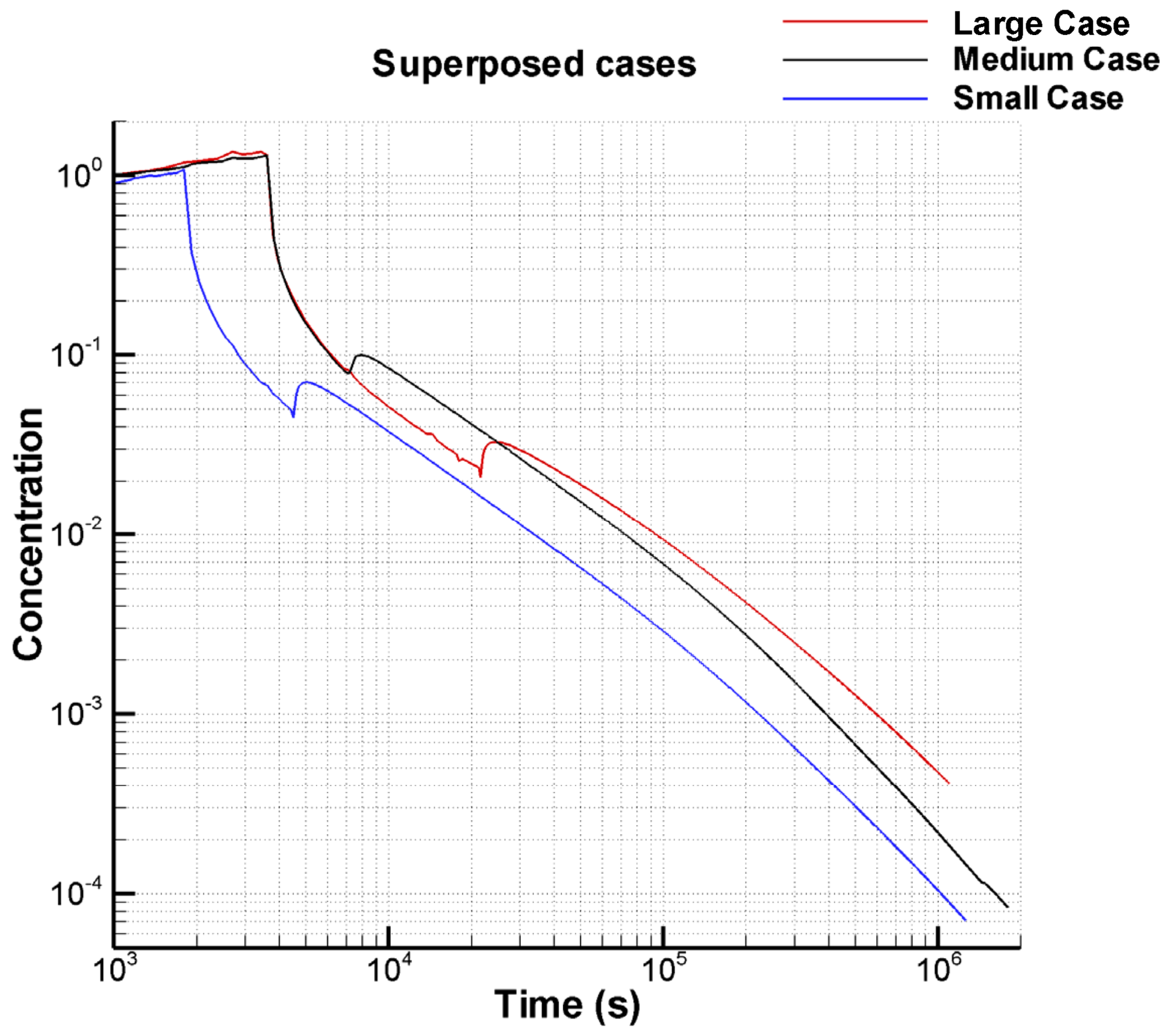


Figure 16: Spatial distribution of the large problem
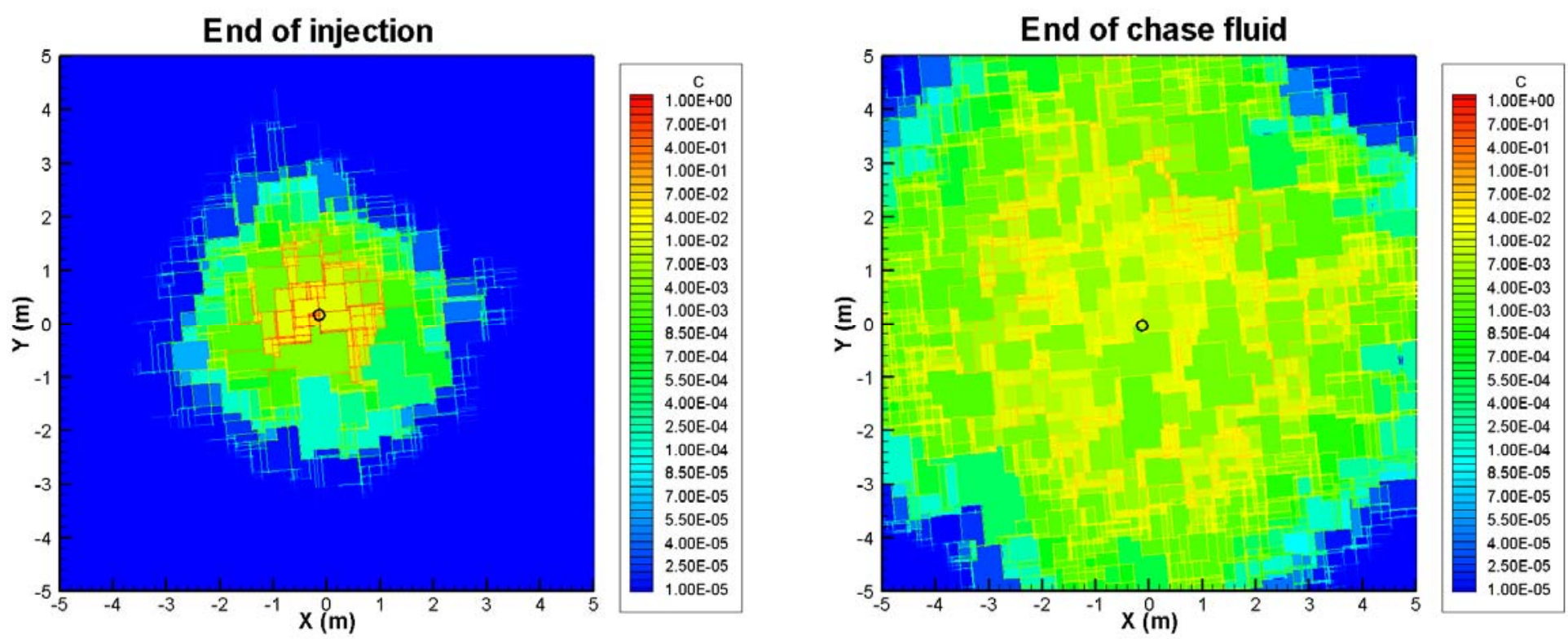

Middle of withdrawal

\section{Large problem}

End of withdrawal
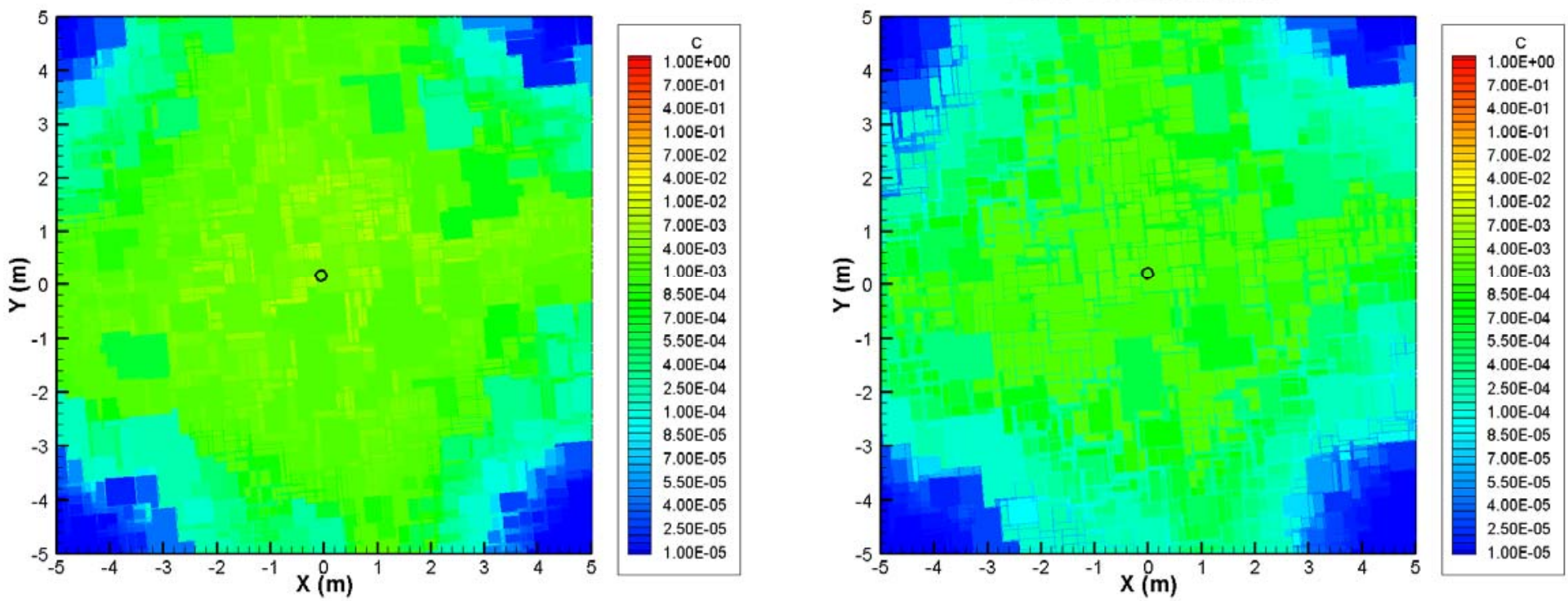
Figure 17: Spatial distribution of the medium problem
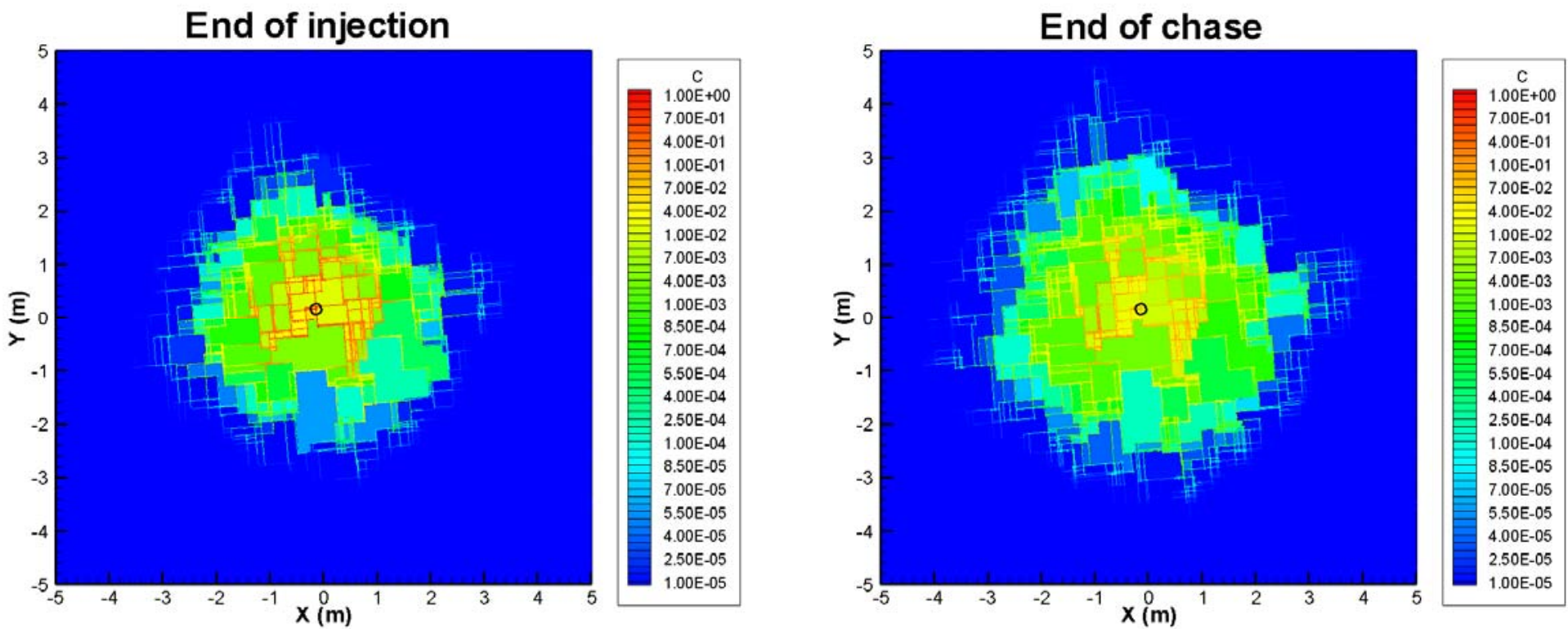

Middle of withdrawal

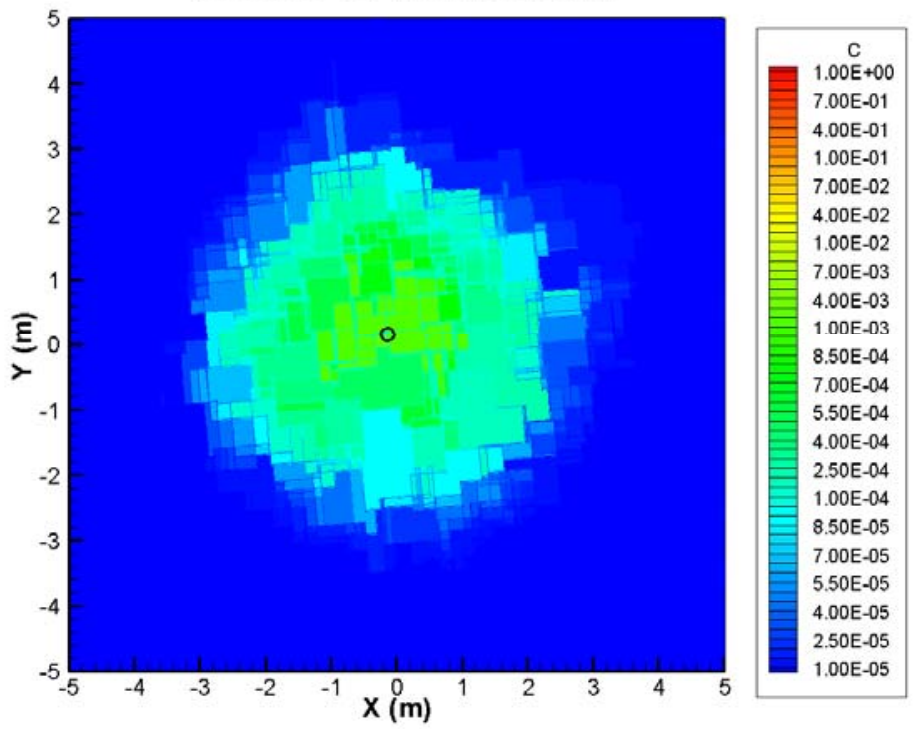

End of withdrawal

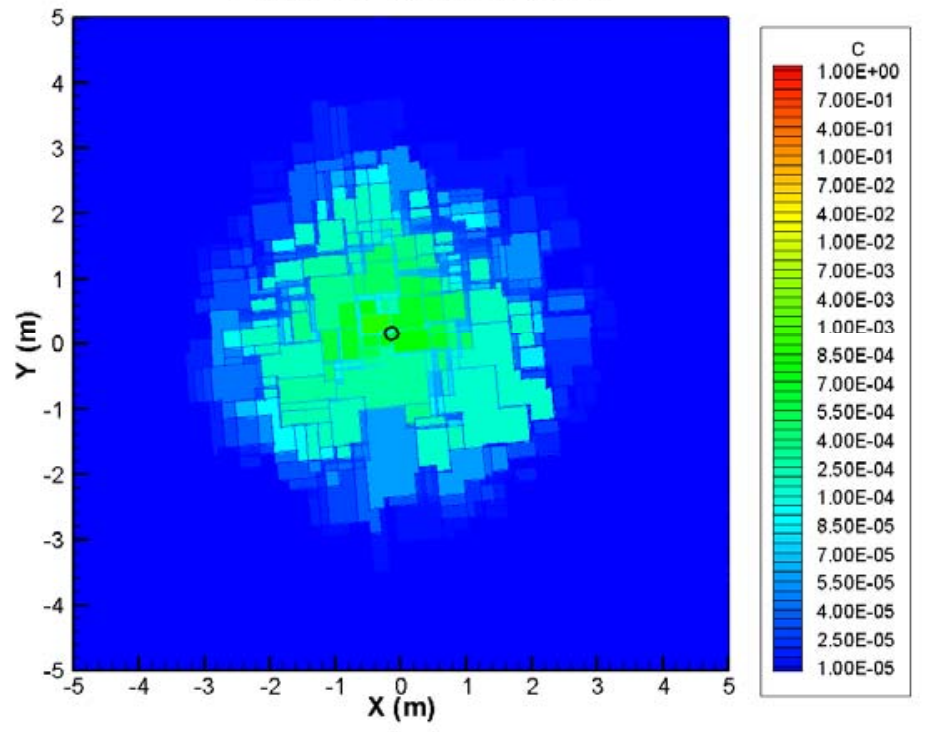


Figure 18: Spatial distribution of the small problem

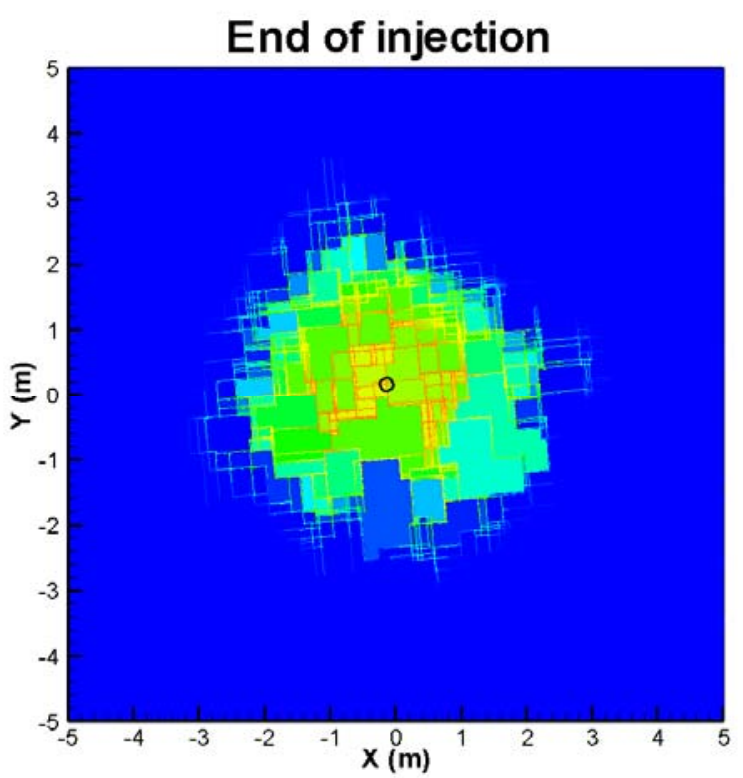

Middle of withdrawal

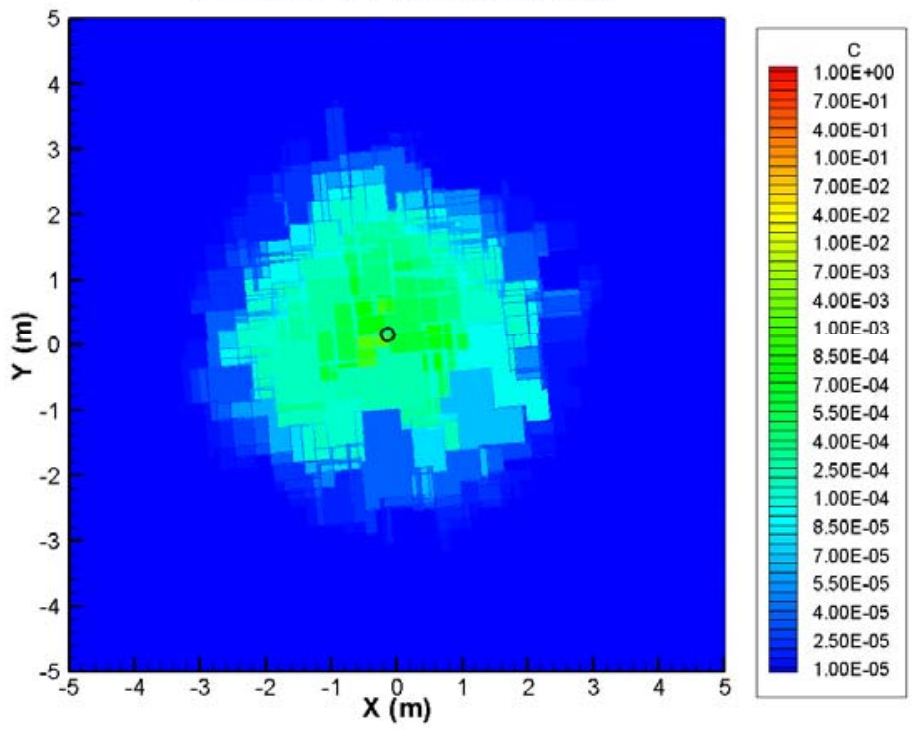

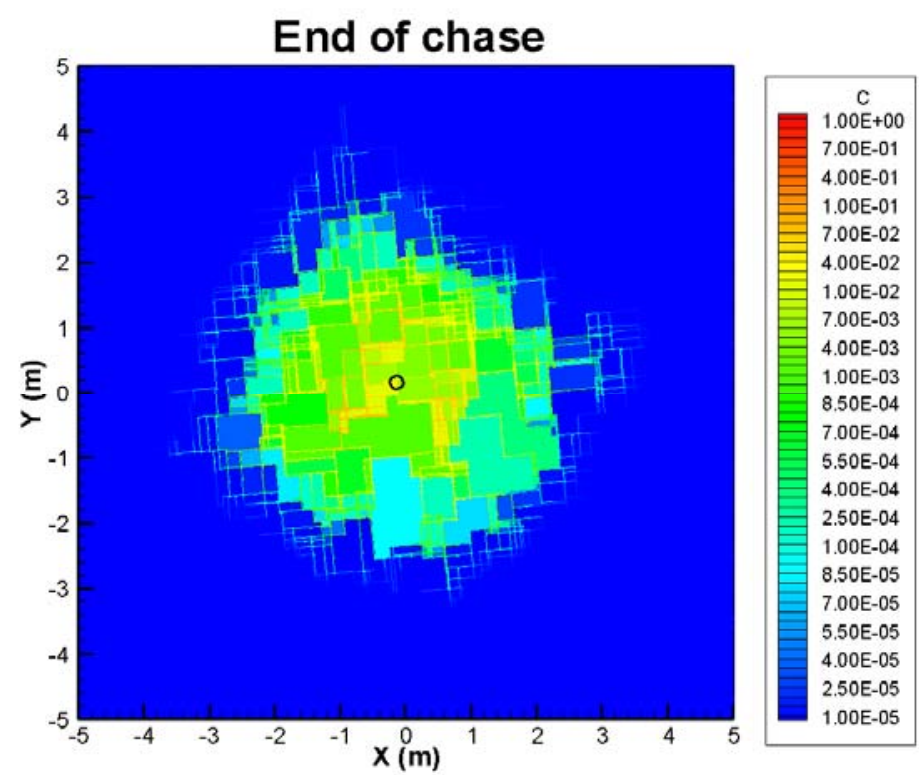

\section{Small problem}

\section{End of withdrawal}

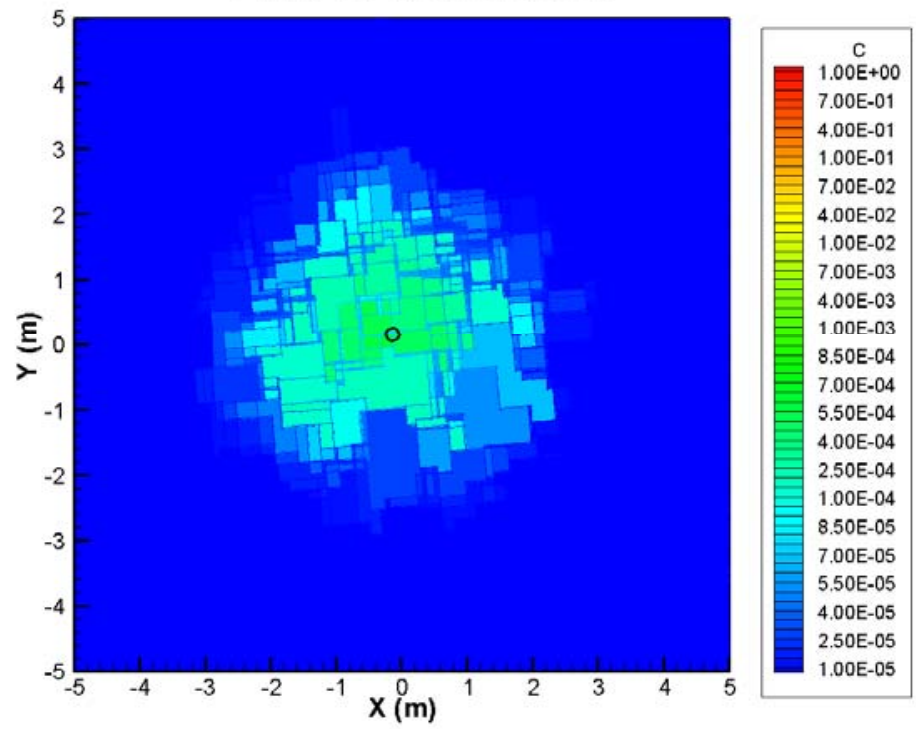


Figure 19: BTCs for porosity (top - log-log scales; bottom - linear-linear scales)

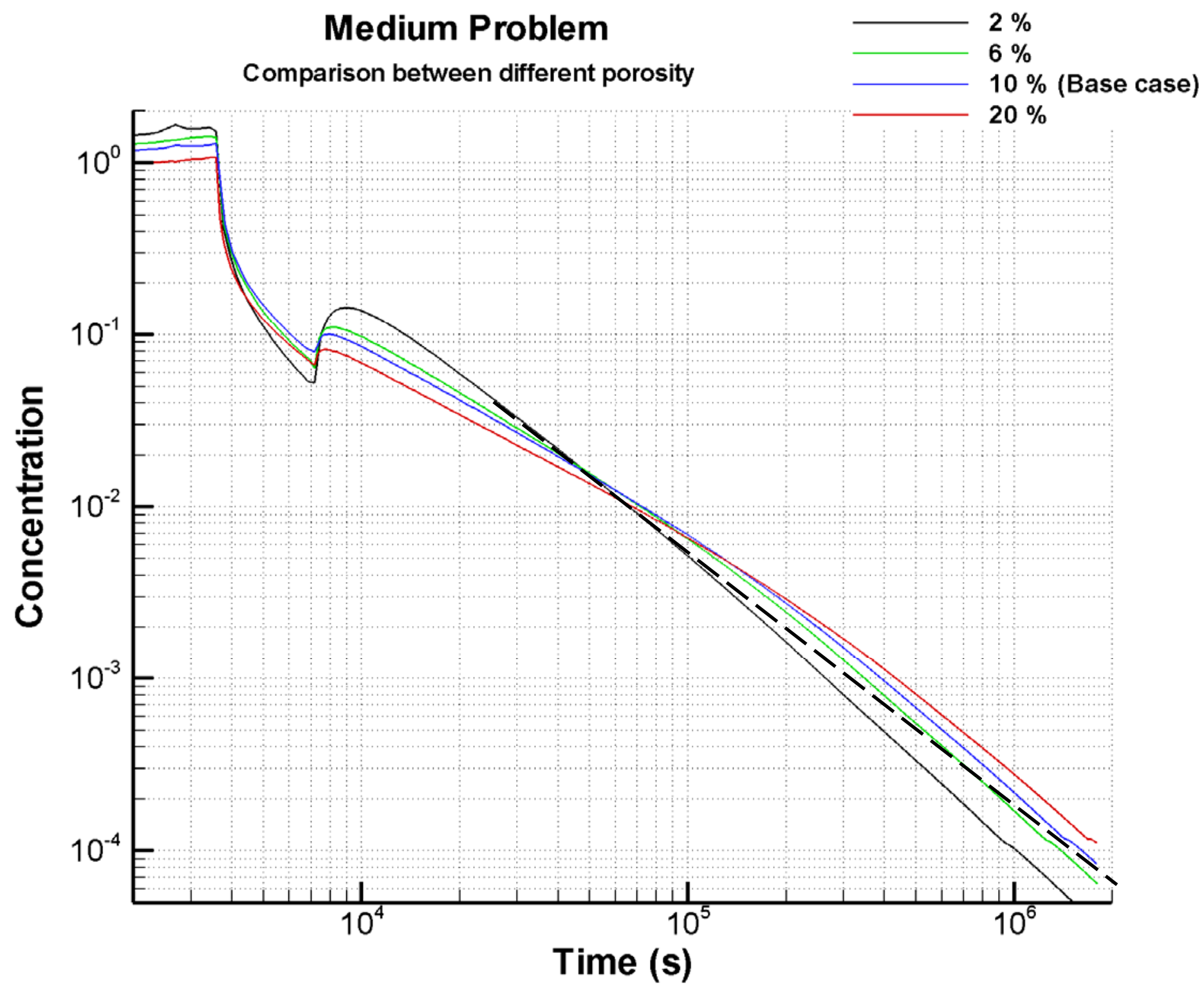

Dashed tail: $-3 / 2$ slope 


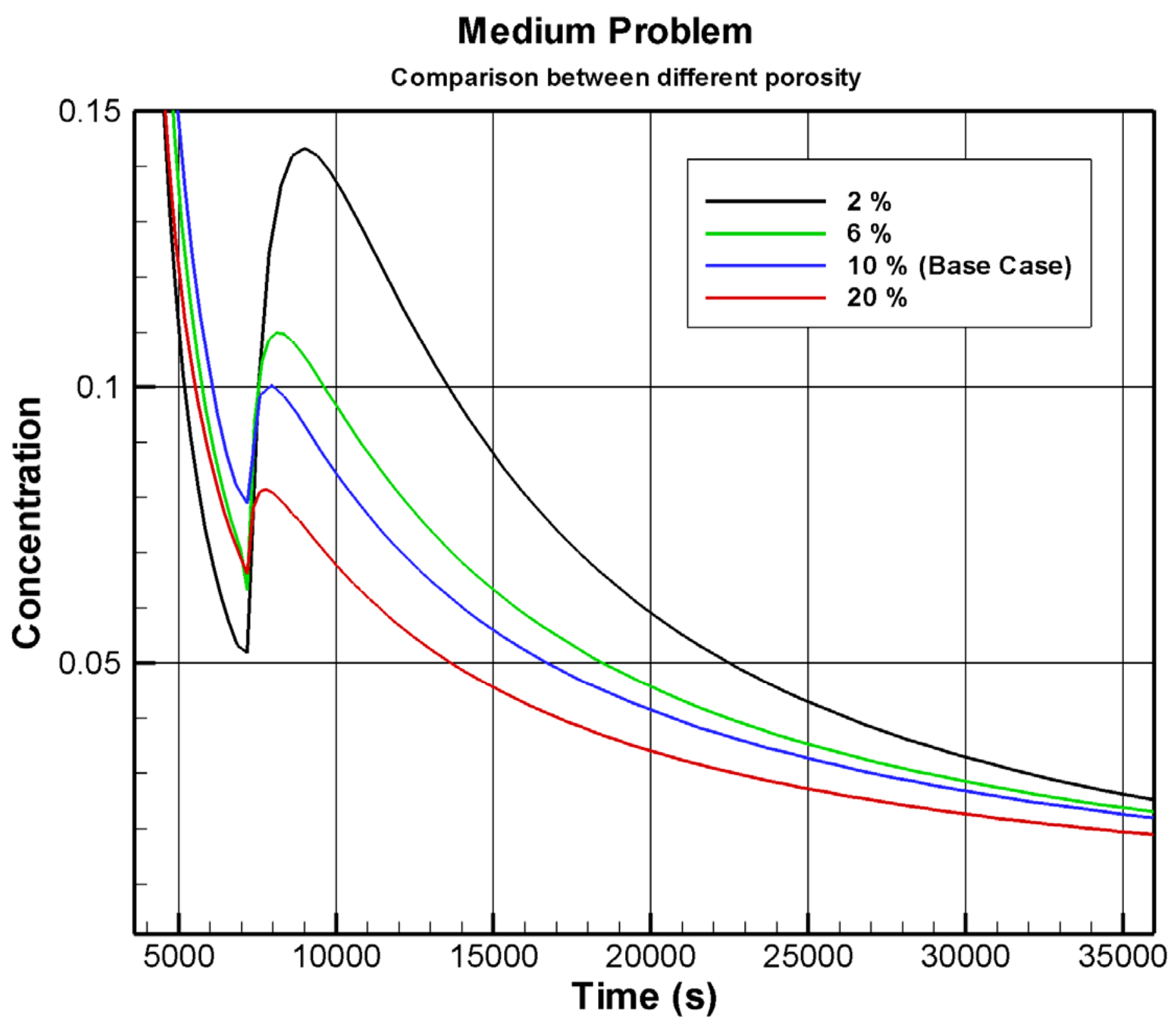


Figure 20: Evolution of spatial distribution for different porosity values
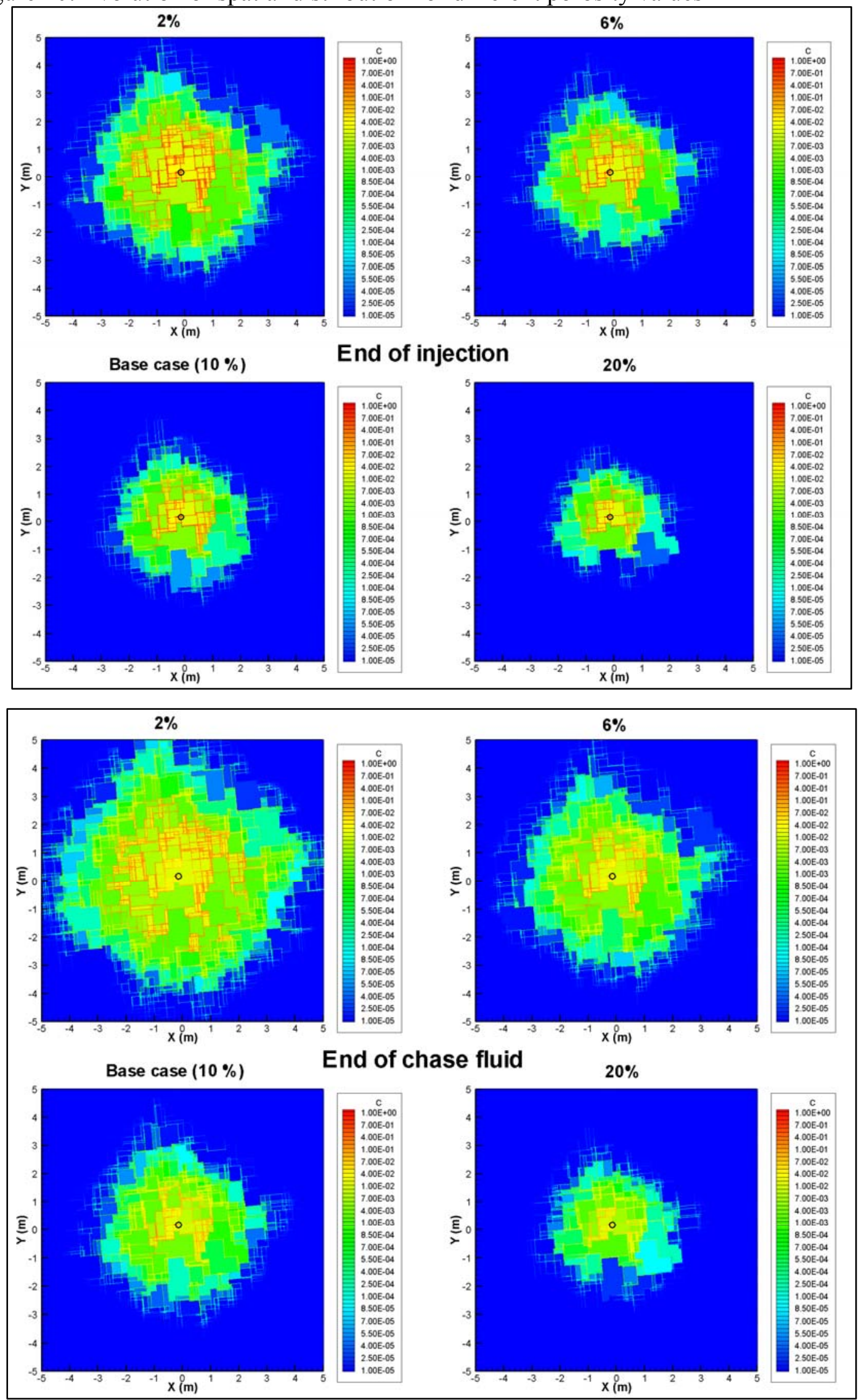

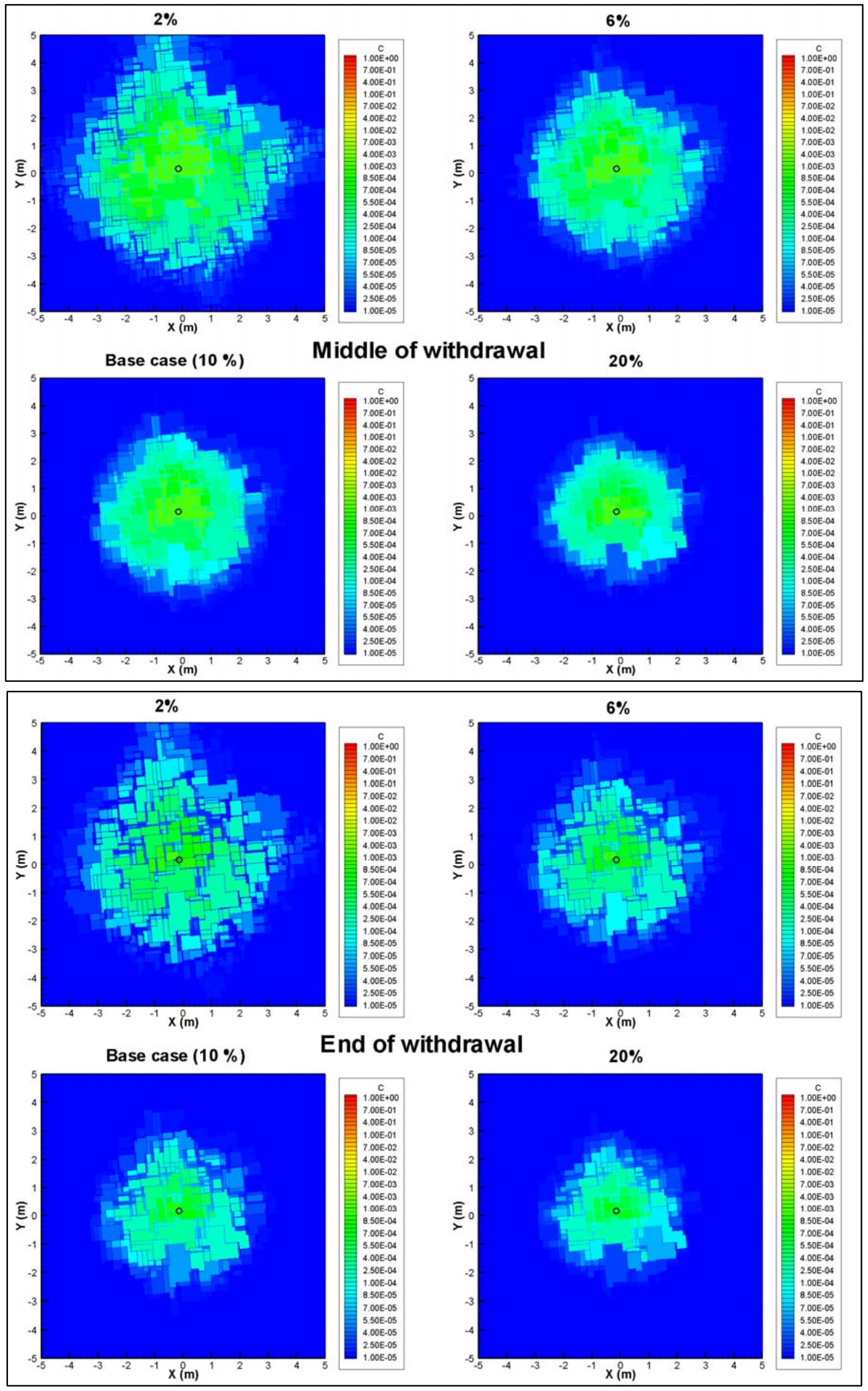
Figure 21: BTCs for diffusion coefficients (top - log-log scales; bottom linear-linear scales)

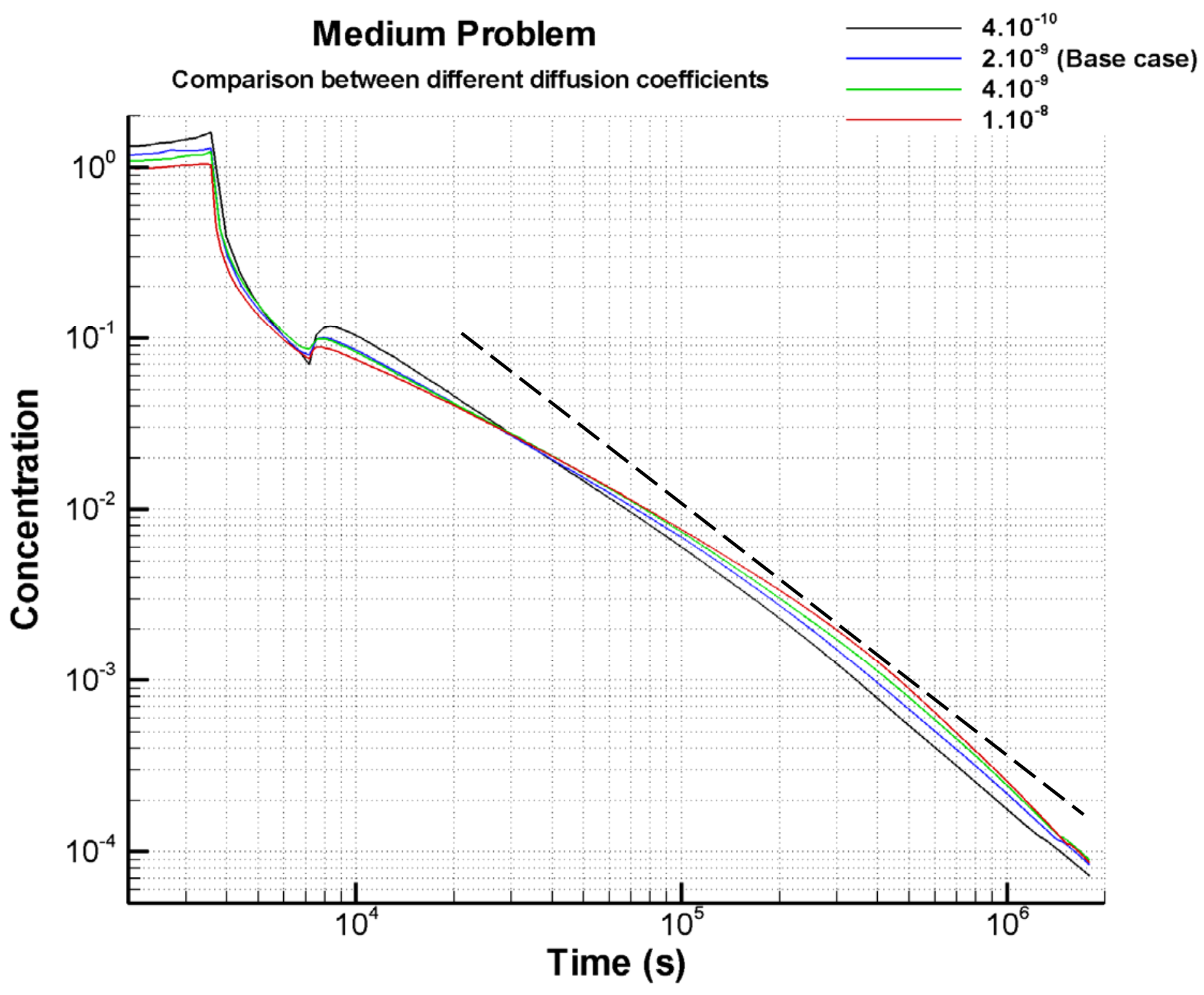

Dashed tail: $-3 / 2$ slope 


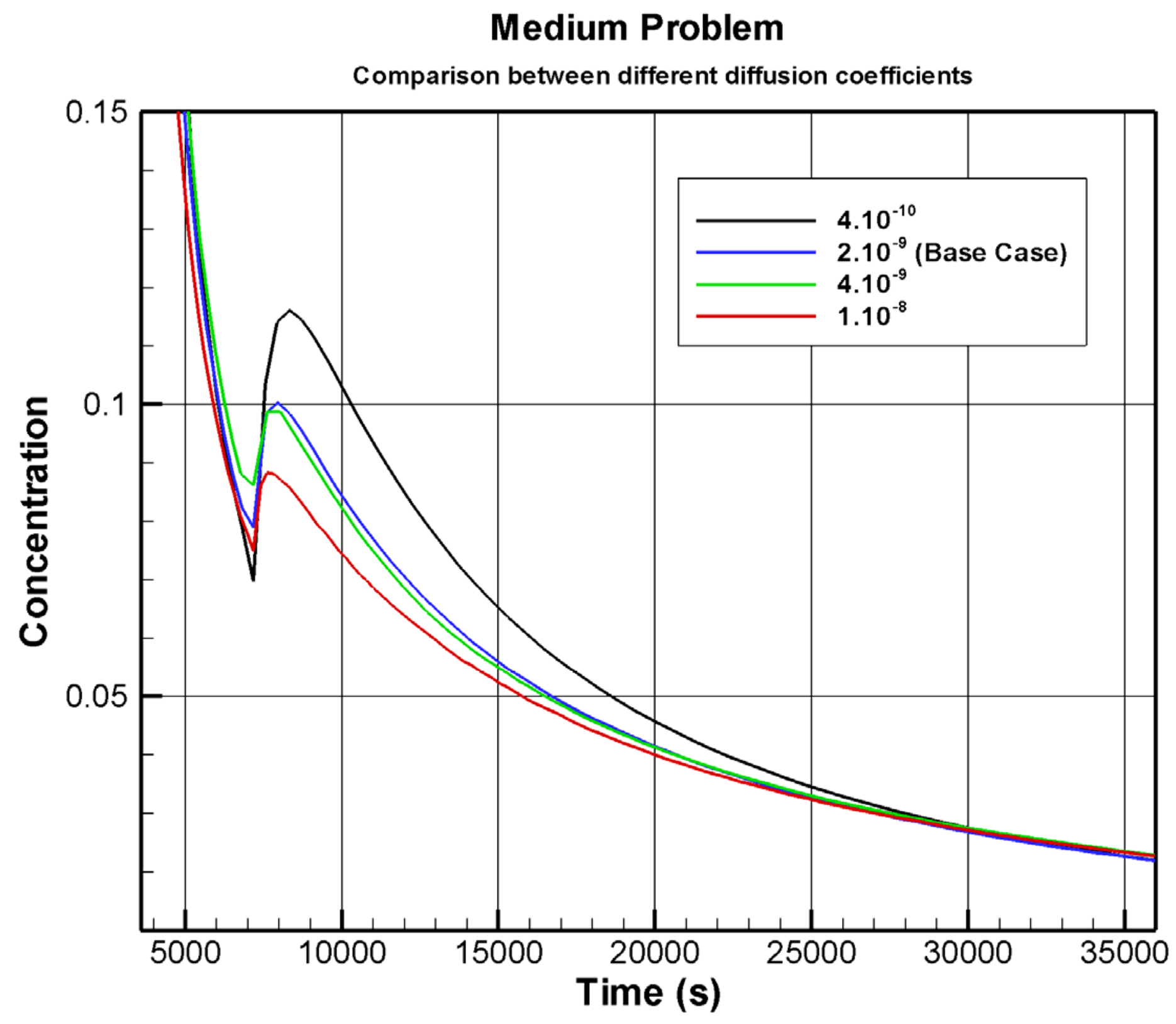


Figure 22: Evolution of spatial distribution for diffusion coefficients
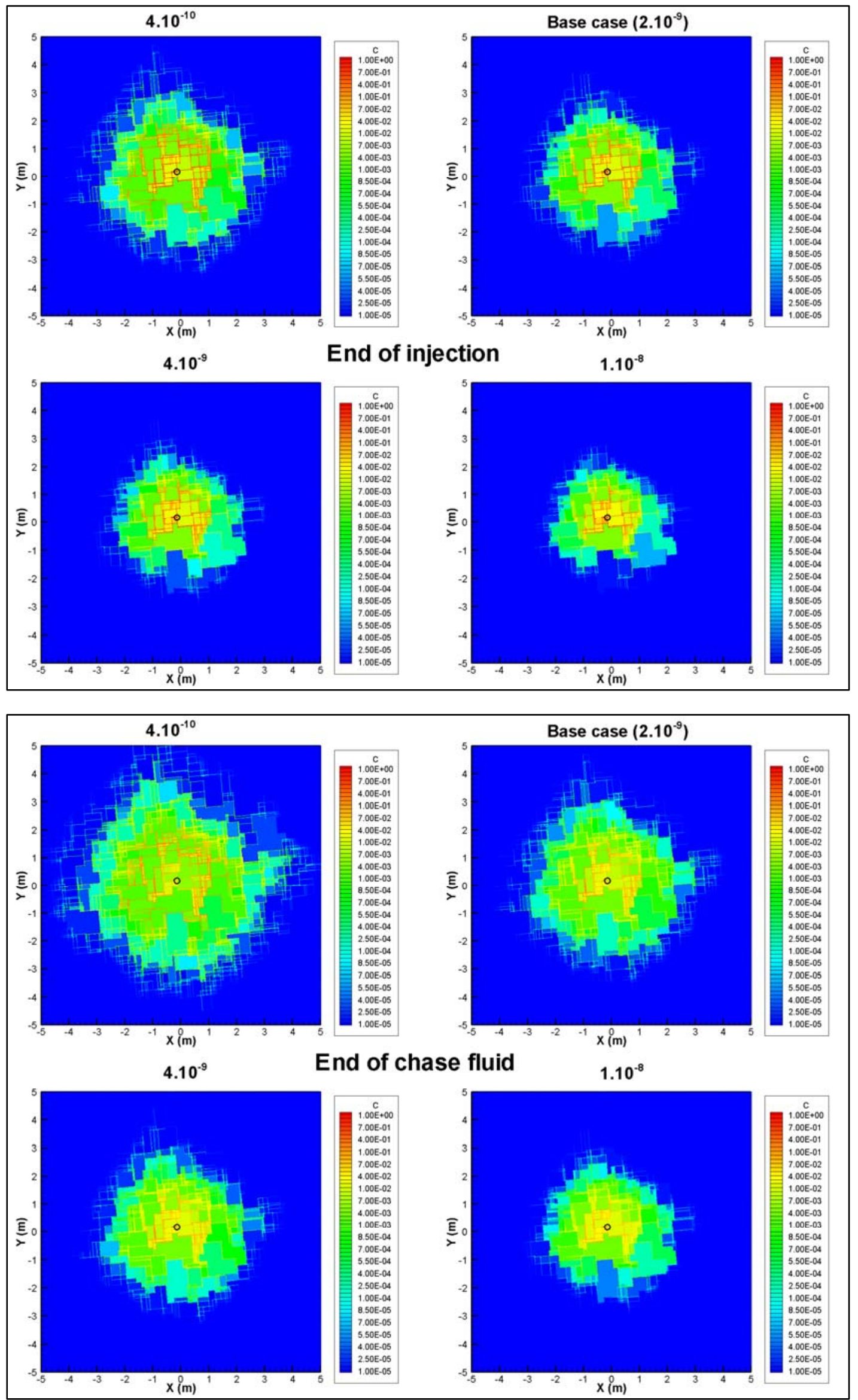

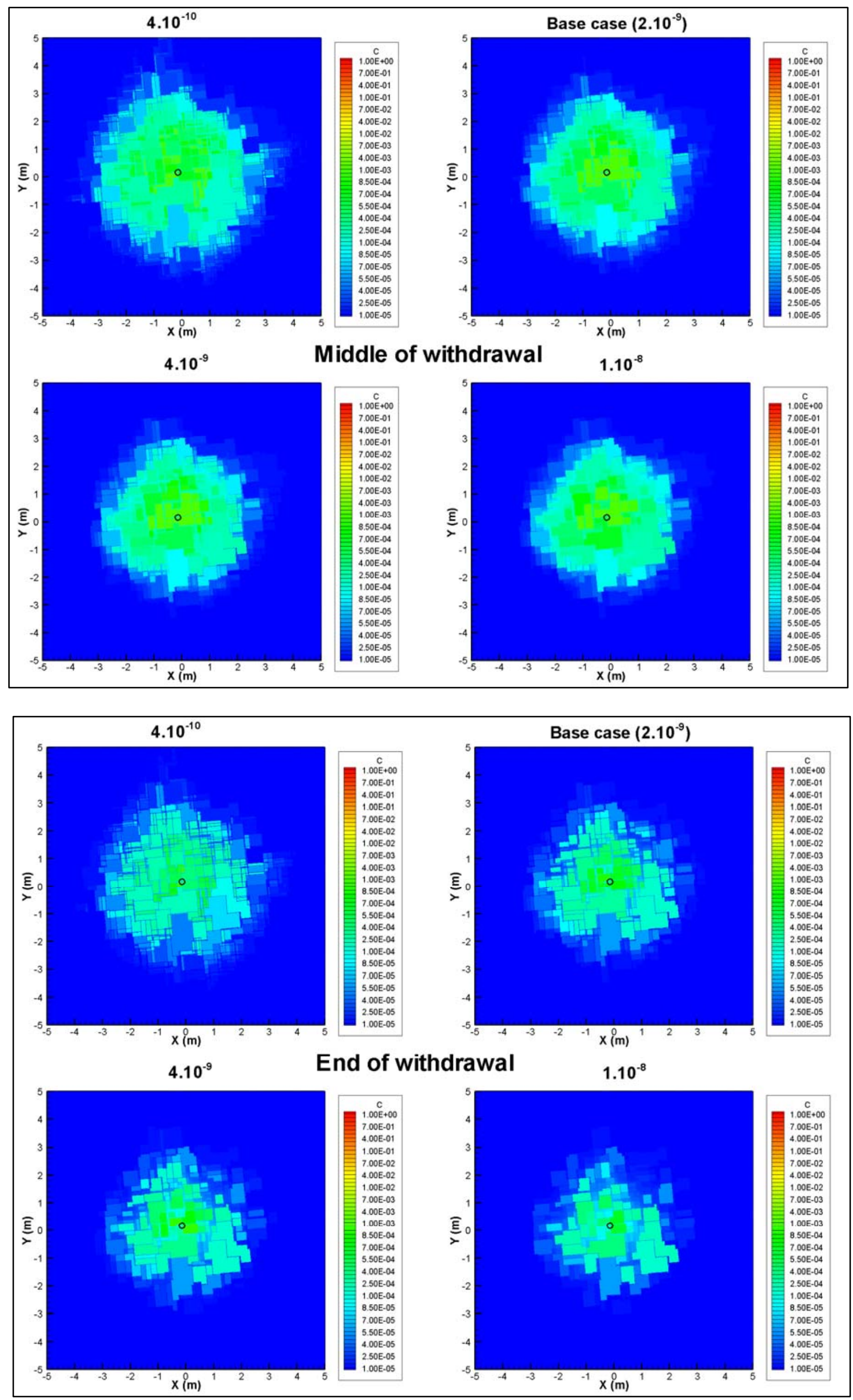
Figure 23: BTCs for retardation coefficients (top - log-log scales; bottom - linear-linear scales)

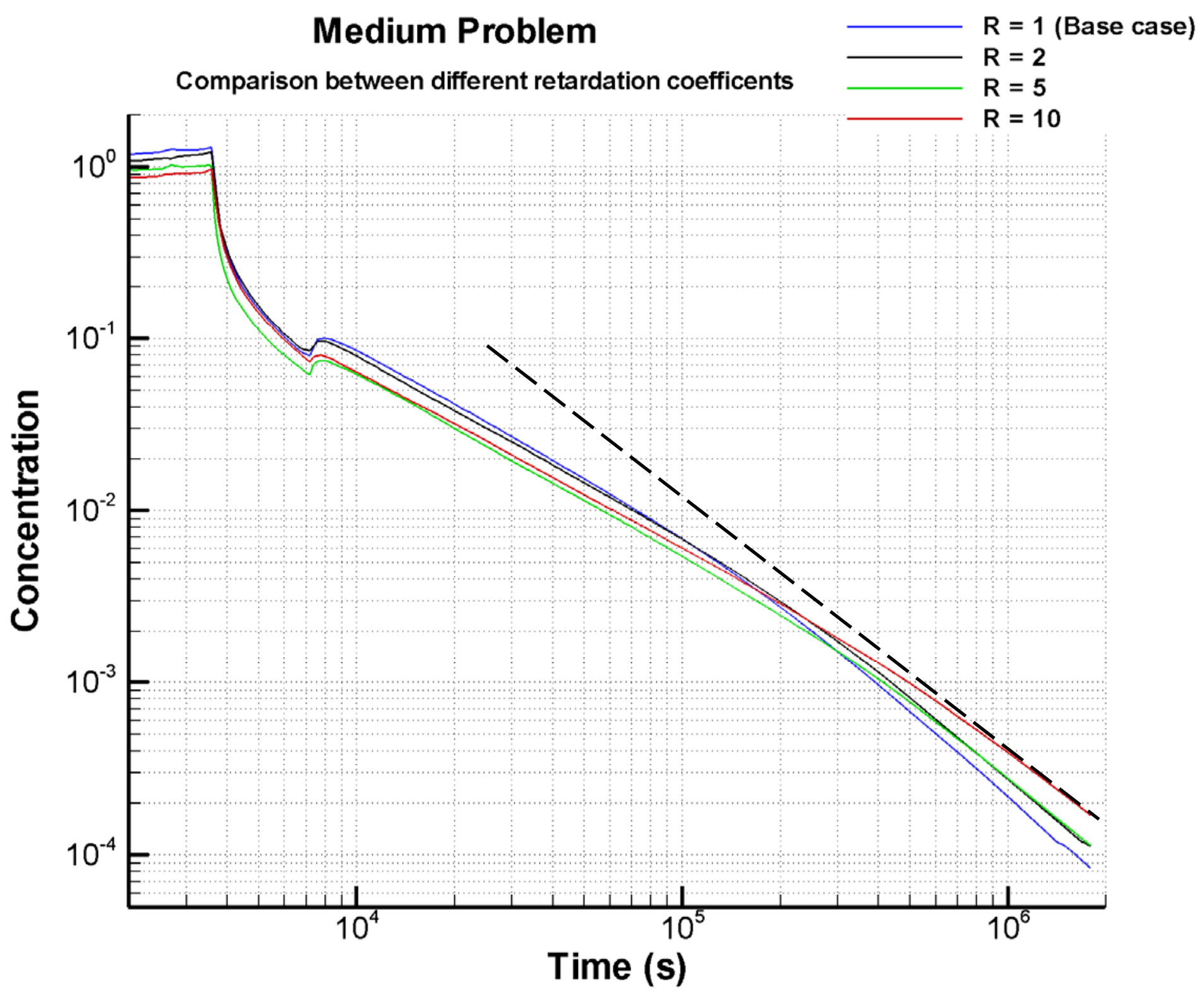

Dashed tail: $-3 / 2$ slope 


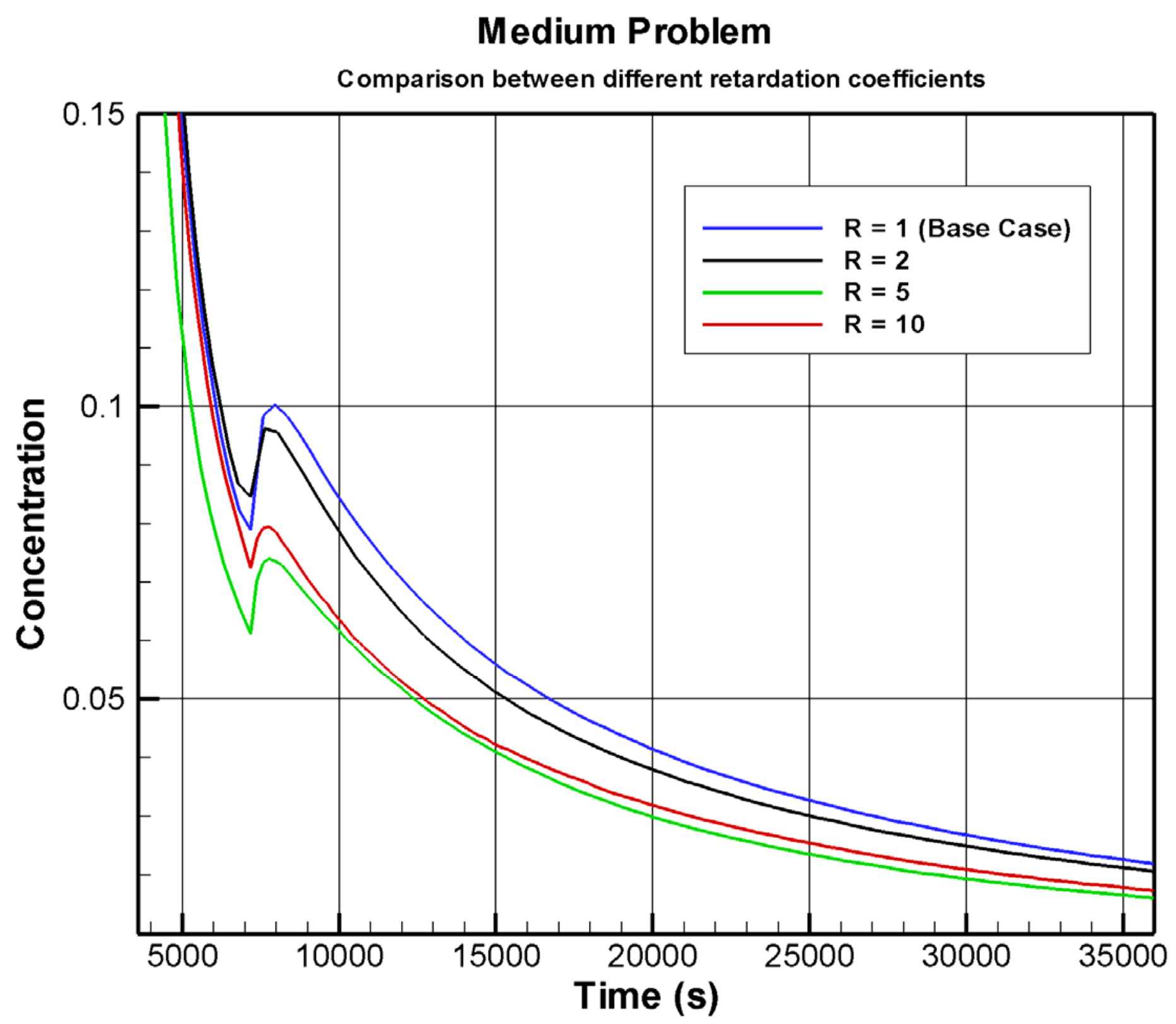


Figure 24: Evolution of spatial distribution for retardation coefficients
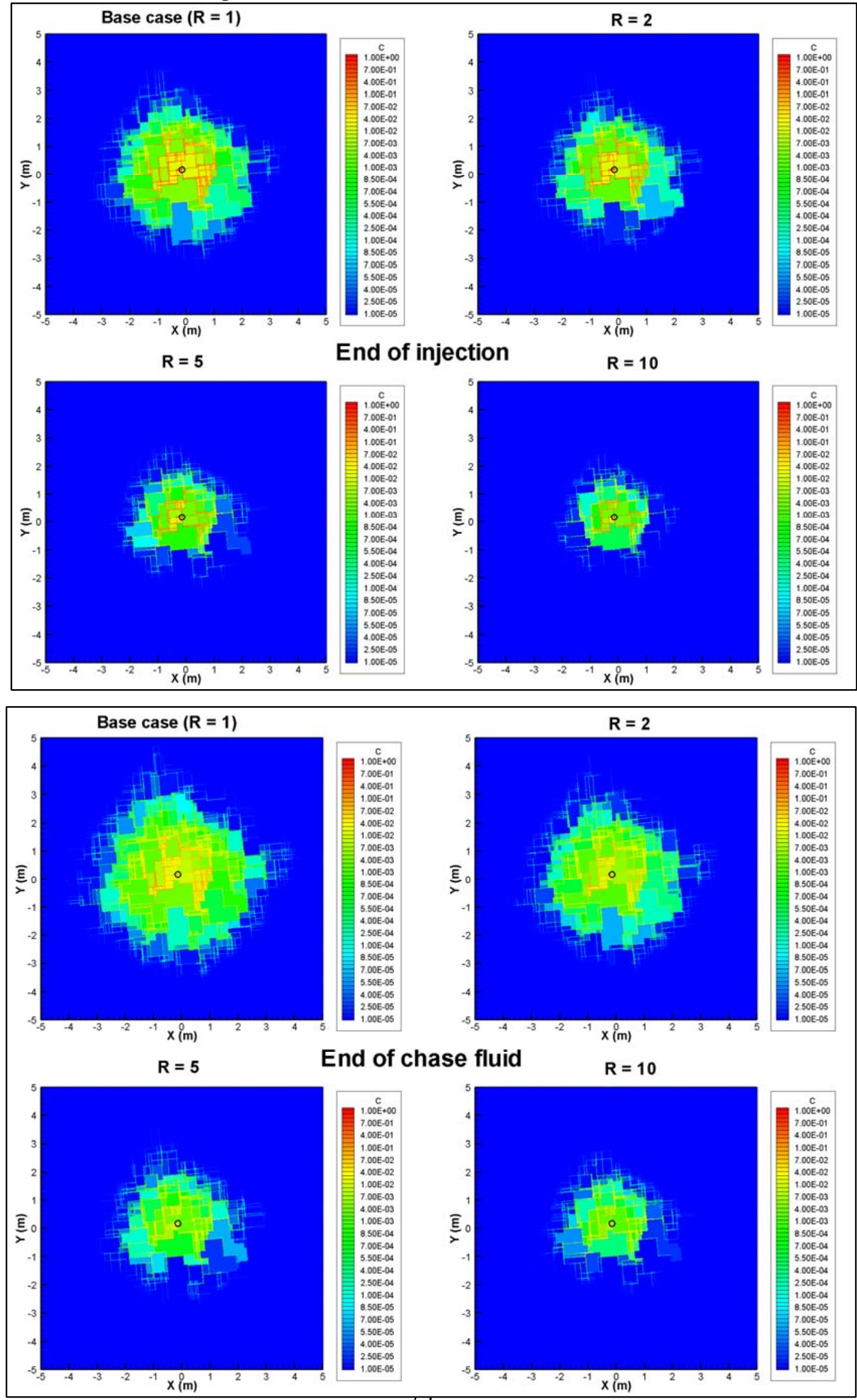

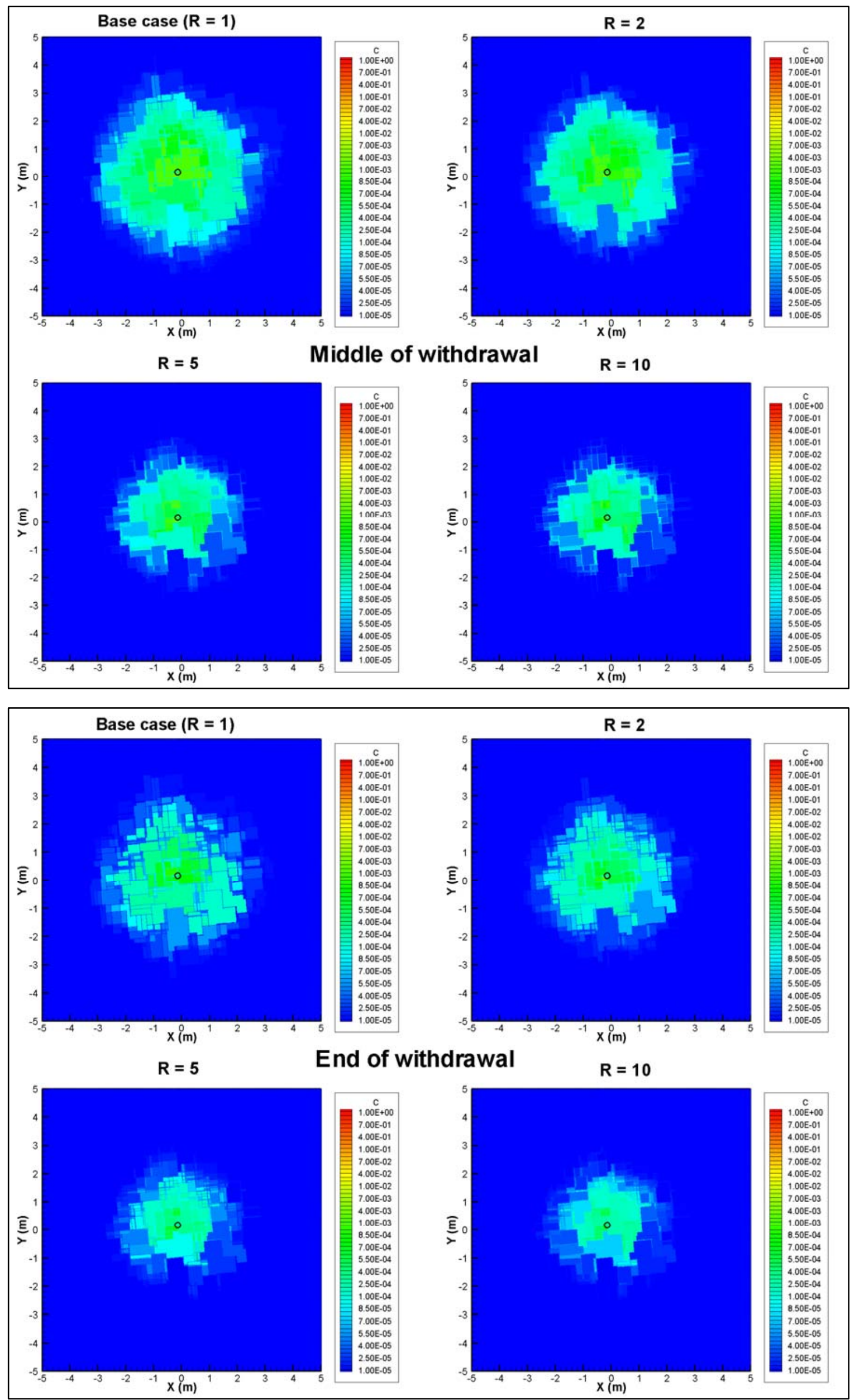
Figure 25: BTCs for apertures (top - log-log scales; bottom - linear-linear scales)

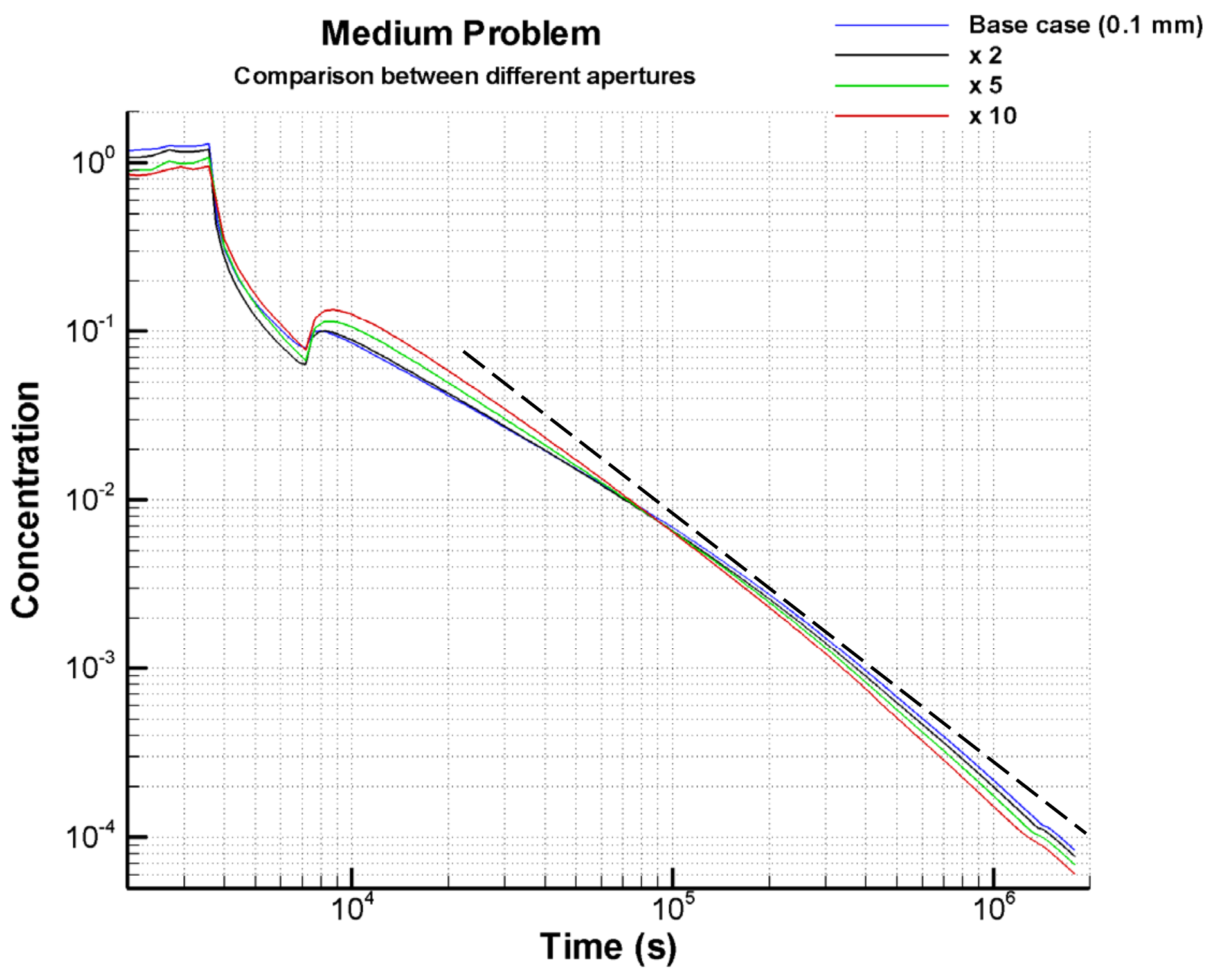

Dashed tail: $-3 / 2$ slope 


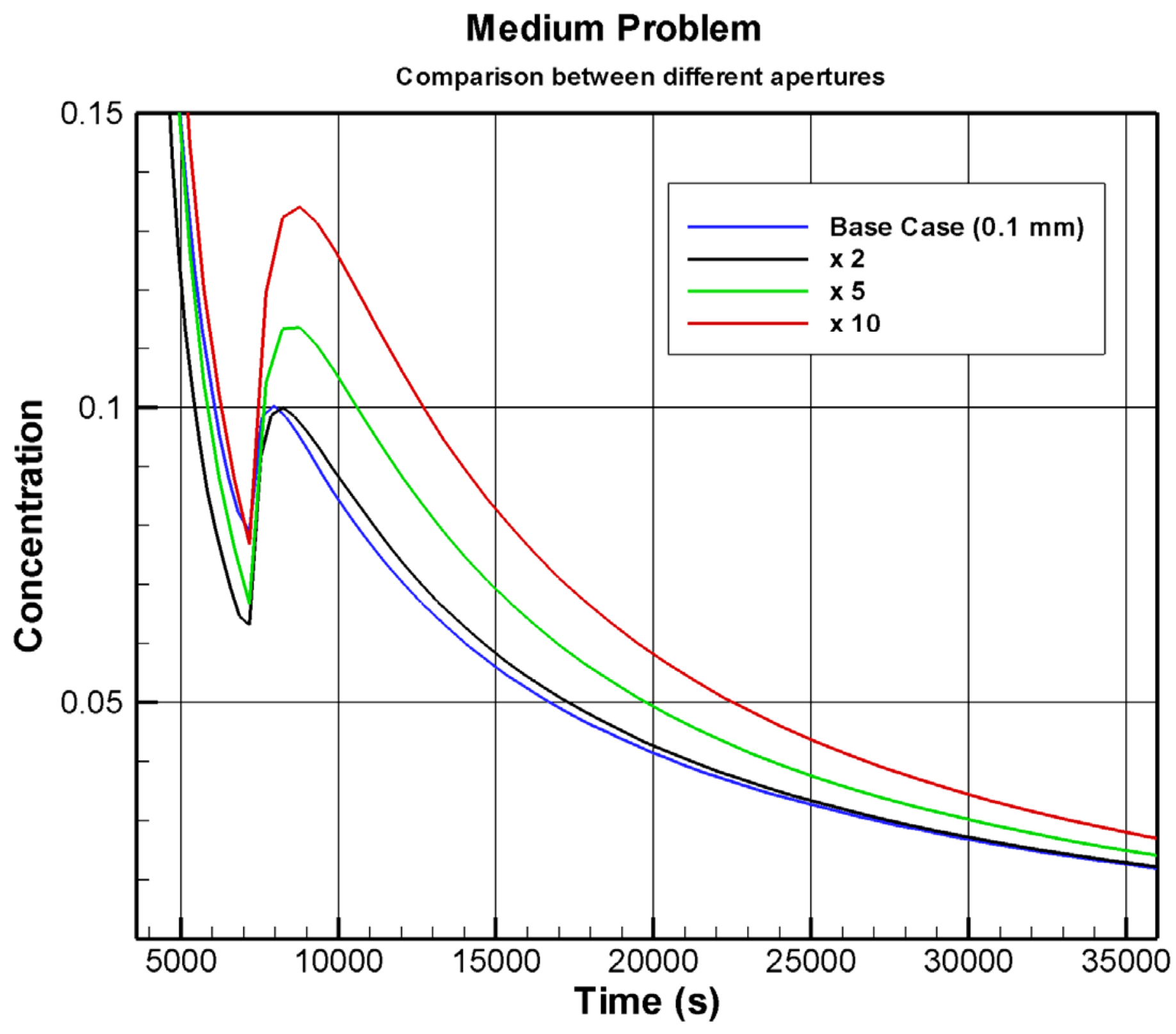


Figure 26: Evolution of spatial distribution for different apertures

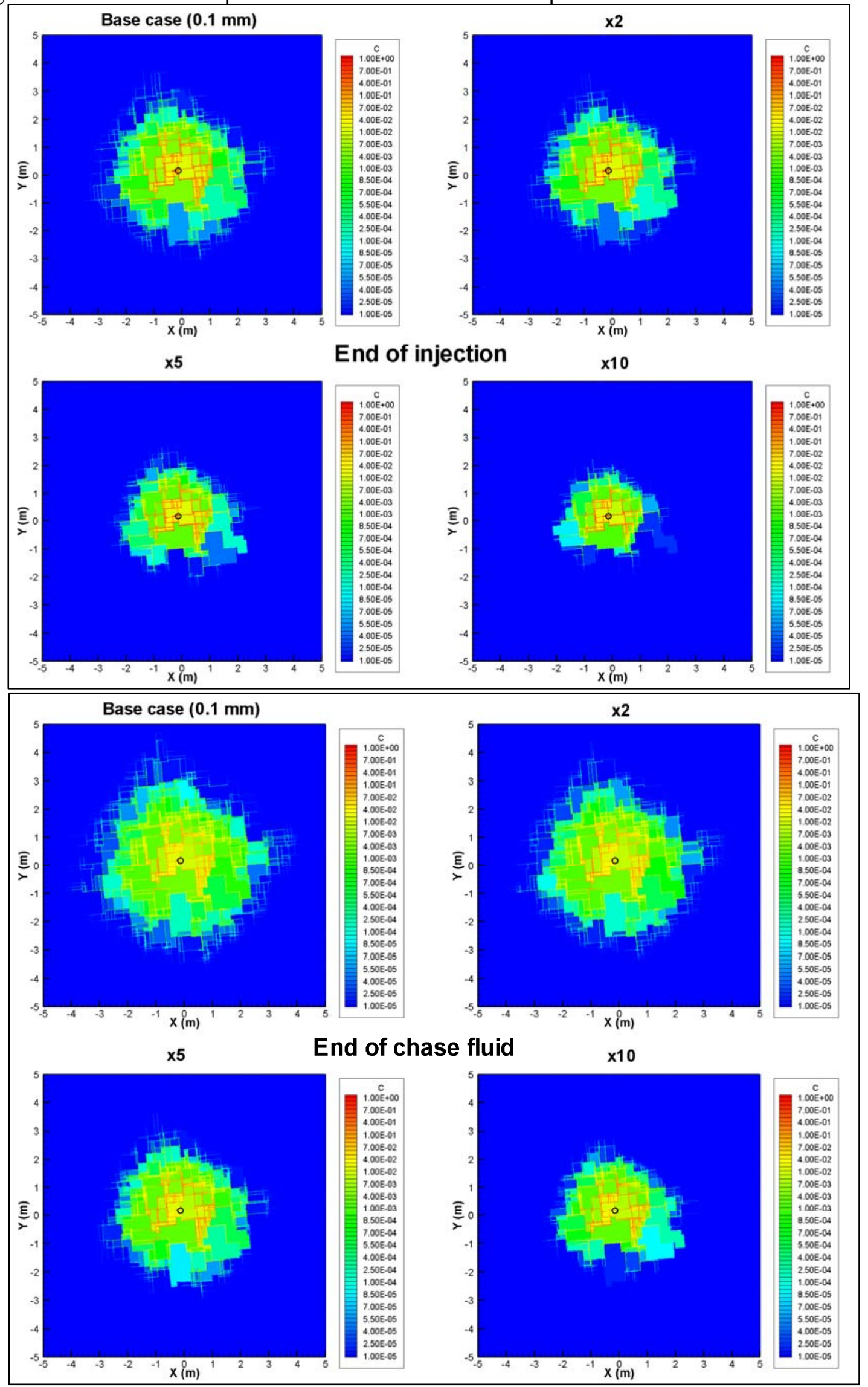



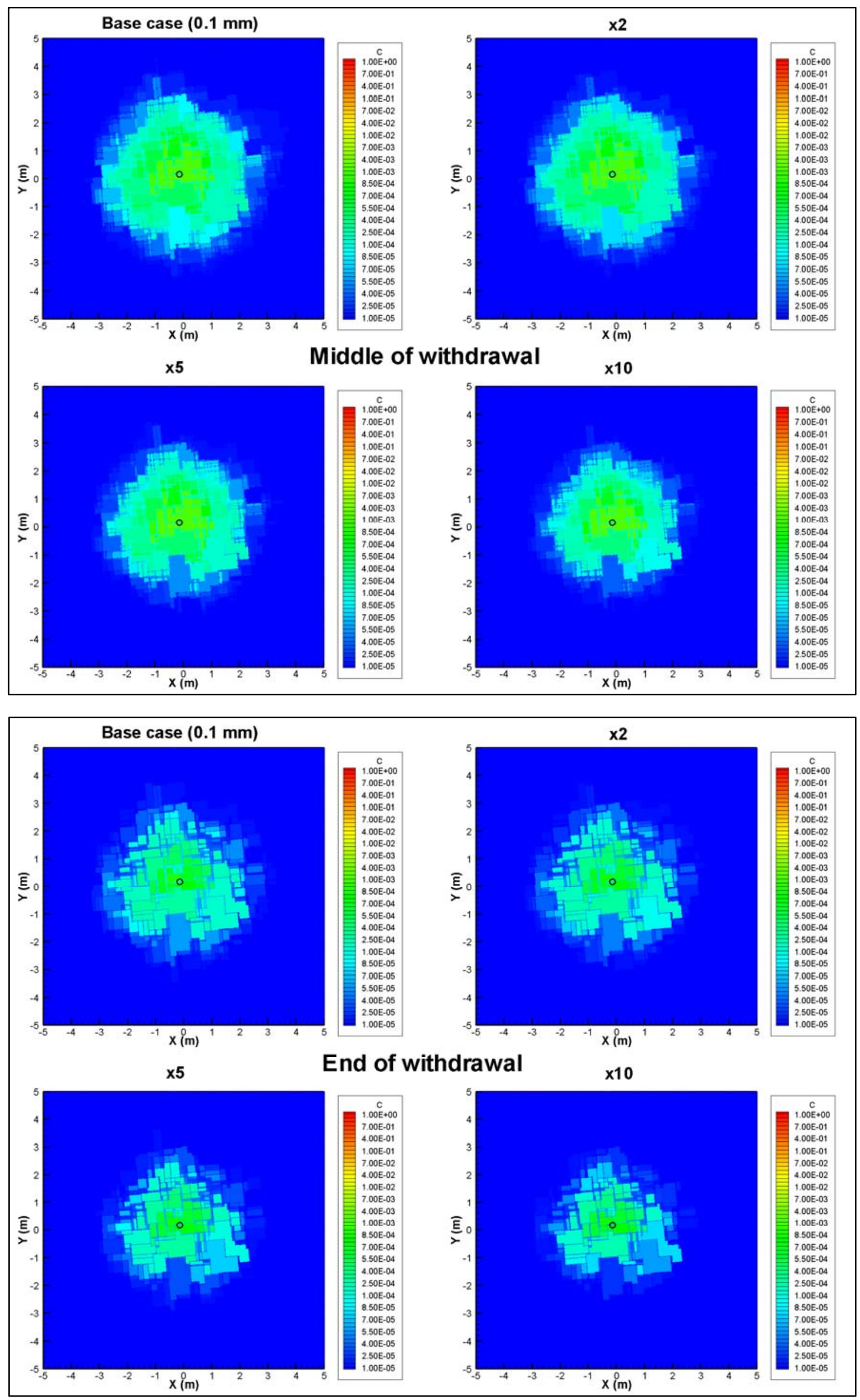
Figure 27: Histograms of the new block size (m) distribution when adding a third set

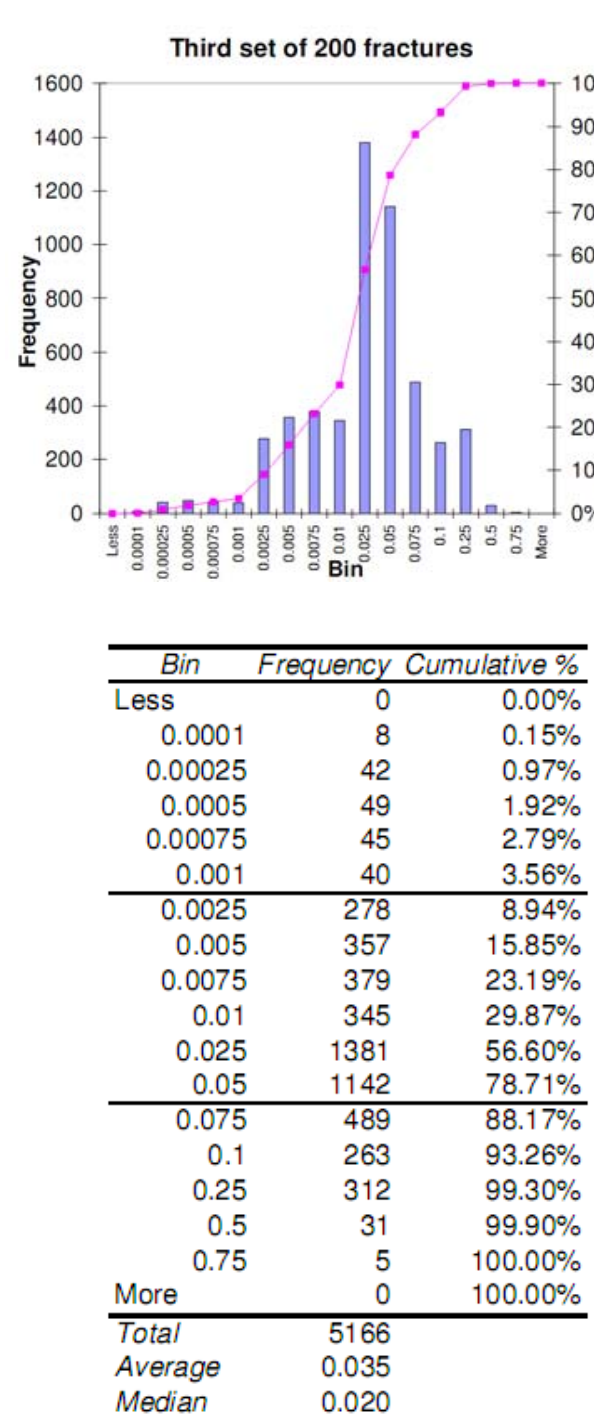

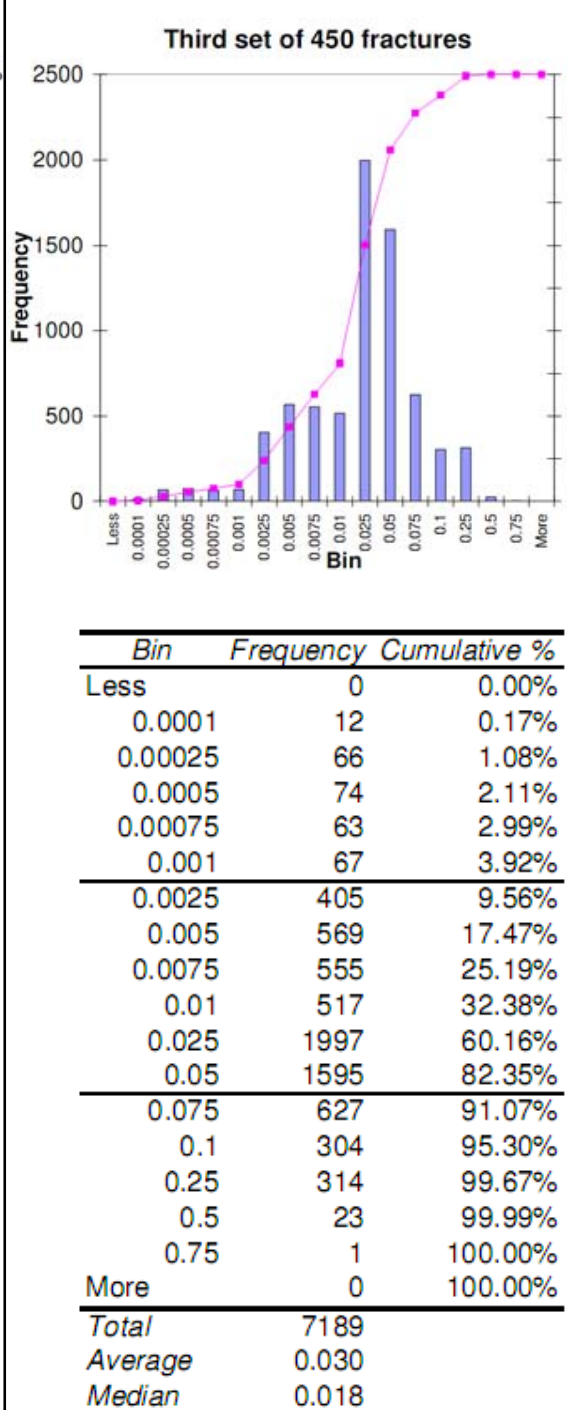

\begin{tabular}{|rrr|}
\hline \multicolumn{1}{|c}{ Bin } & Frequency & Cumulative $\%$ \\
\hline Less & 0 & $0.00 \%$ \\
0.0001 & 1 & $0.03 \%$ \\
0.00025 & 29 & $0.88 \%$ \\
0.0005 & 32 & $1.81 \%$ \\
0.00075 & 29 & $2.66 \%$ \\
0.001 & 21 & $3.27 \%$ \\
\hline 0.0025 & 159 & $7.91 \%$ \\
0.005 & 212 & $14.09 \%$ \\
0.0075 & 228 & $20.75 \%$ \\
0.01 & 209 & $26.85 \%$ \\
0.025 & 860 & $51.94 \%$ \\
0.05 & 740 & $73.53 \%$ \\
\hline 0.075 & 368 & $84.27 \%$ \\
0.1 & 204 & $90.22 \%$ \\
0.25 & 292 & $98.75 \%$ \\
0.5 & 37 & $99.82 \%$ \\
0.75 & 6 & $100.00 \%$ \\
More & 0 & $100.00 \%$ \\
\hline Total & 3427 & \\
Average & 0.042 & \\
Median & 0.024 & \\
\hline
\end{tabular}

Reminder: Base Case

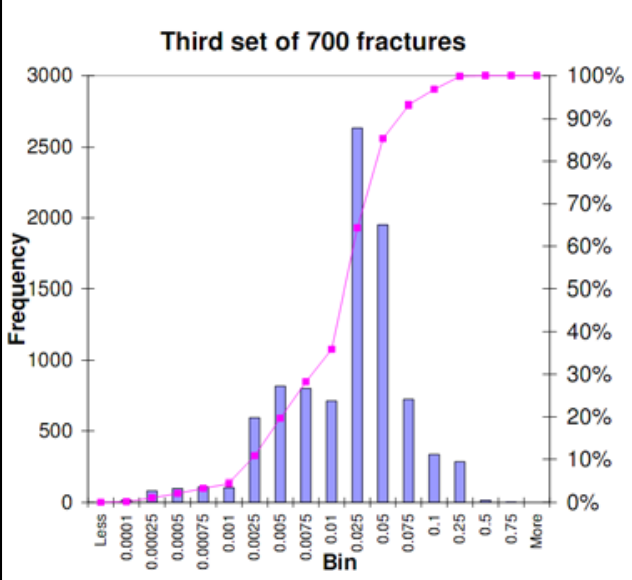

\begin{tabular}{rrr}
\hline \multicolumn{1}{c}{ Bin } & Frequency & Cumulative $\%$ \\
\hline Less & 0 & $0.00 \%$ \\
0.0001 & 17 & $0.18 \%$ \\
0.00025 & 82 & $1.07 \%$ \\
0.0005 & 98 & $2.12 \%$ \\
0.00075 & 109 & $3.30 \%$ \\
0.001 & 105 & $4.43 \%$ \\
\hline 0.0025 & 593 & $10.82 \%$ \\
0.005 & 816 & $19.62 \%$ \\
0.0075 & 800 & $28.25 \%$ \\
0.01 & 712 & $35.92 \%$ \\
0.025 & 2634 & $64.32 \%$ \\
0.05 & 1951 & $85.36 \%$ \\
\hline 0.075 & 724 & $93.16 \%$ \\
0.1 & 334 & $96.77 \%$ \\
0.25 & 283 & $99.82 \%$ \\
0.5 & 16 & $99.99 \%$ \\
0.75 & 1 & $100.00 \%$ \\
More & 0 & $100.00 \%$ \\
\hline Total & 9275 & \\
Average & 0.026 & \\
Median & 0.016 &
\end{tabular}


Figure 28: BTCs for $3^{\text {rd }}$ sets (top - log-log scales; bottom - linear-linear scales)

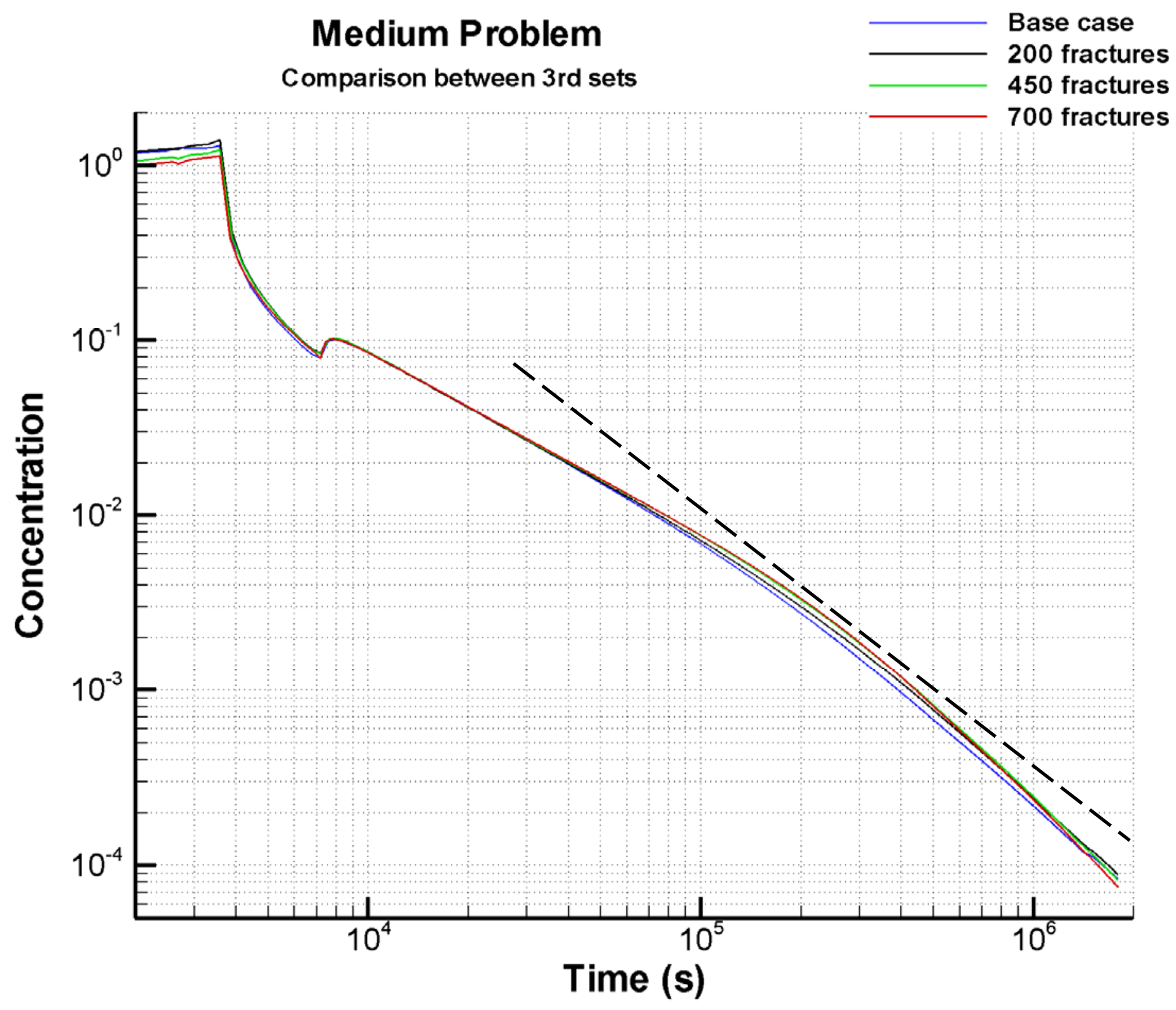

Dashed tail: $-3 / 2$ slope 


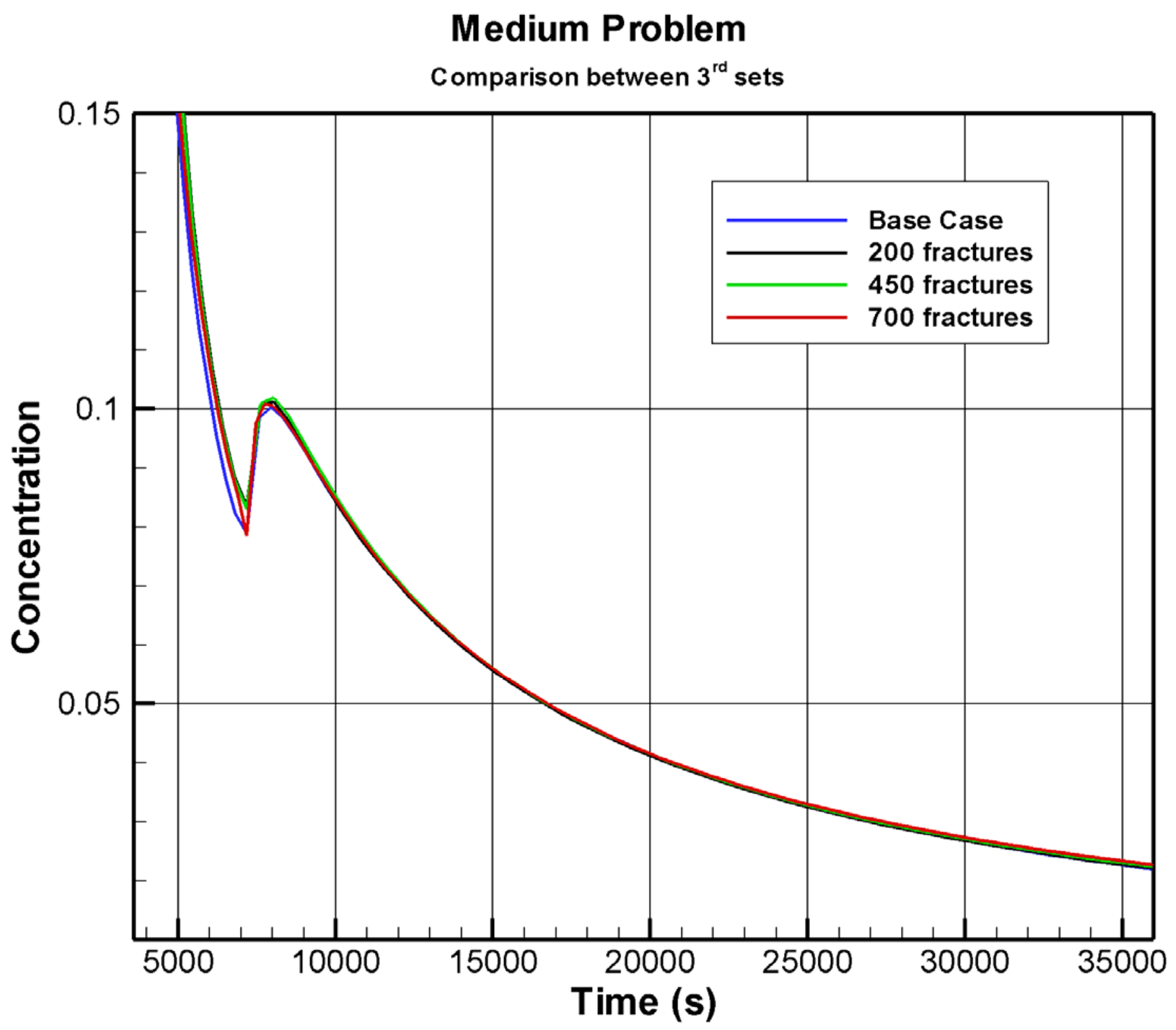


Figure 29: Evolution of spatial distribution for adding a $3^{\text {rd }}$ set
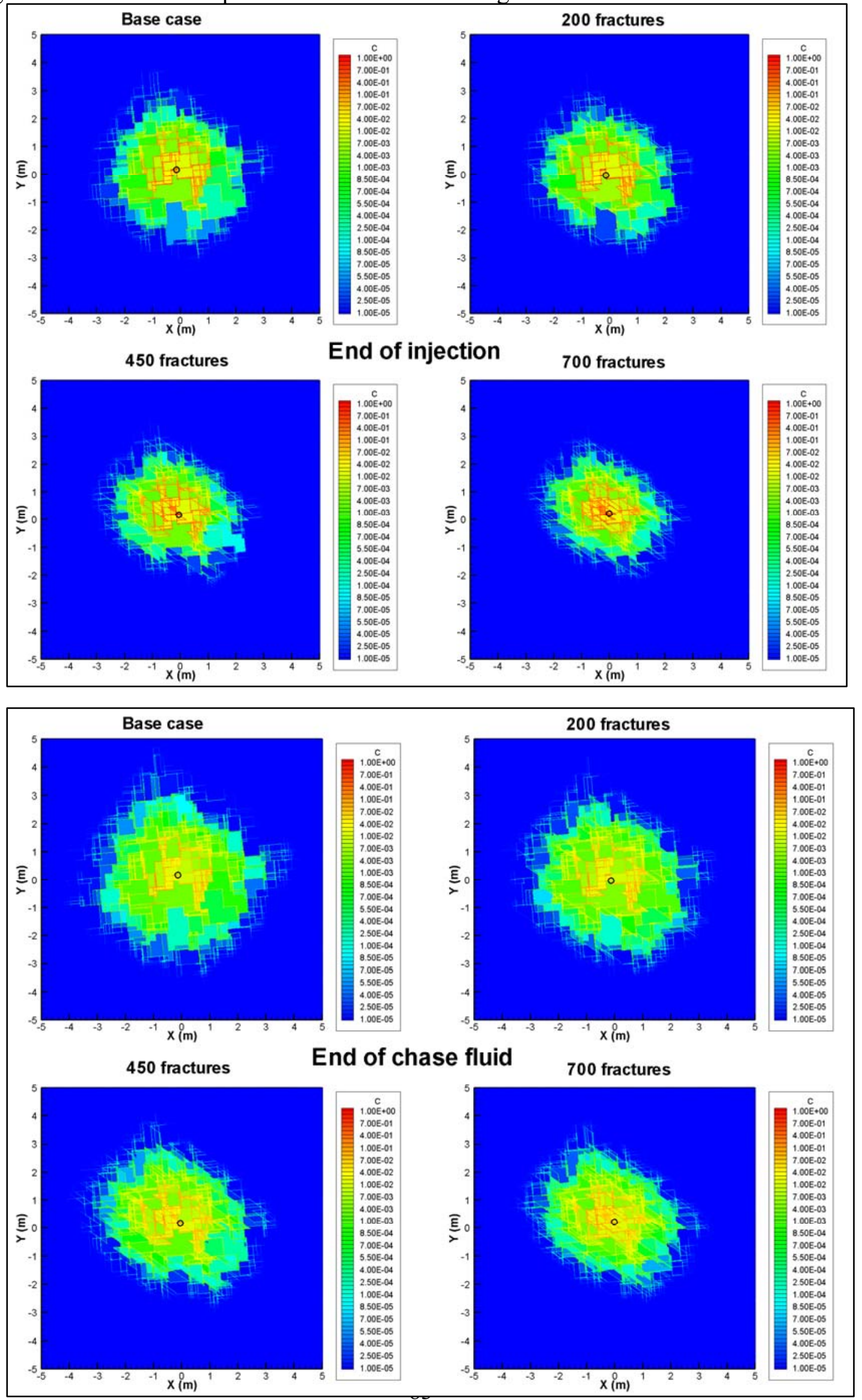

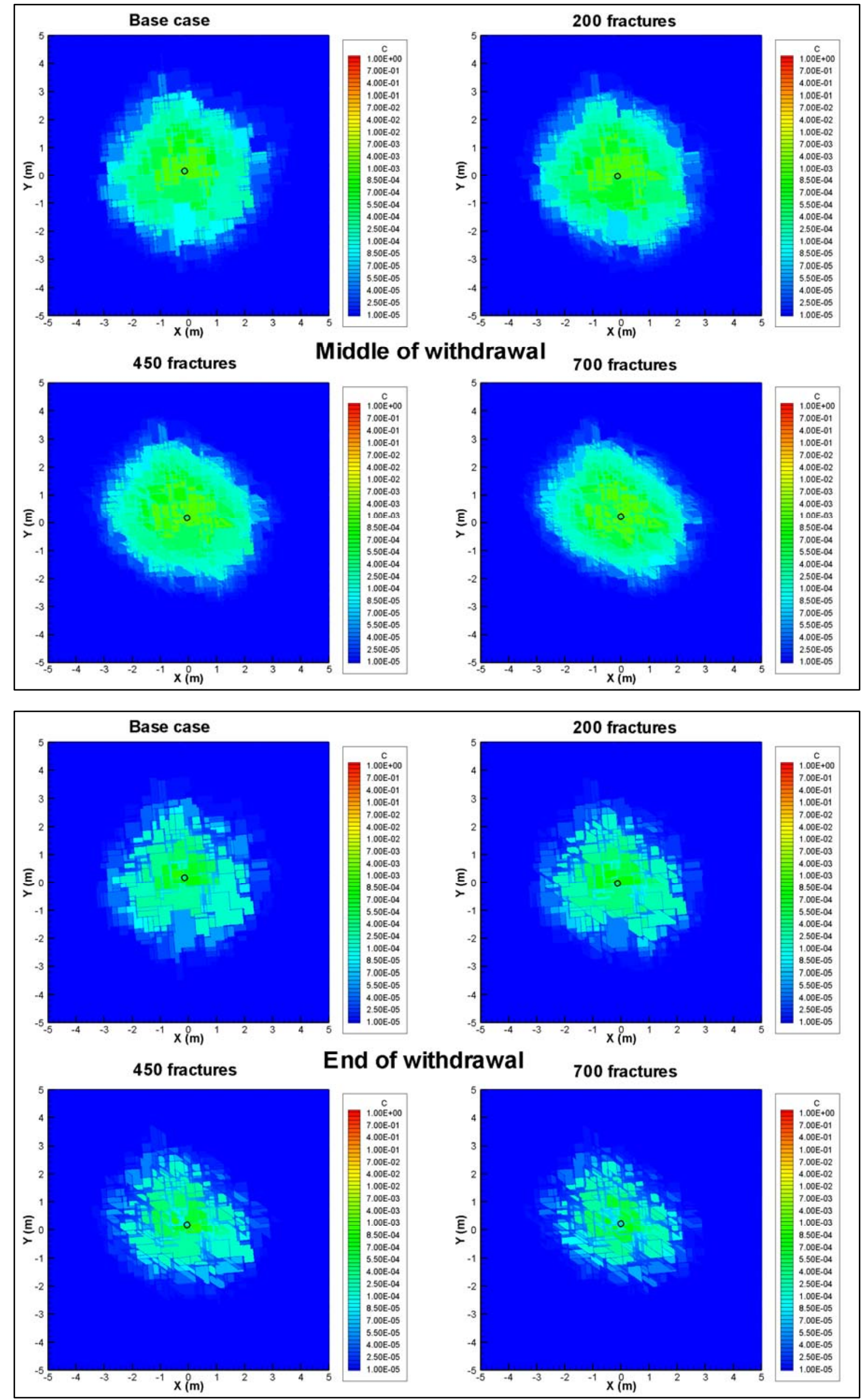
Figure 30: BTC for small aperture enlargements

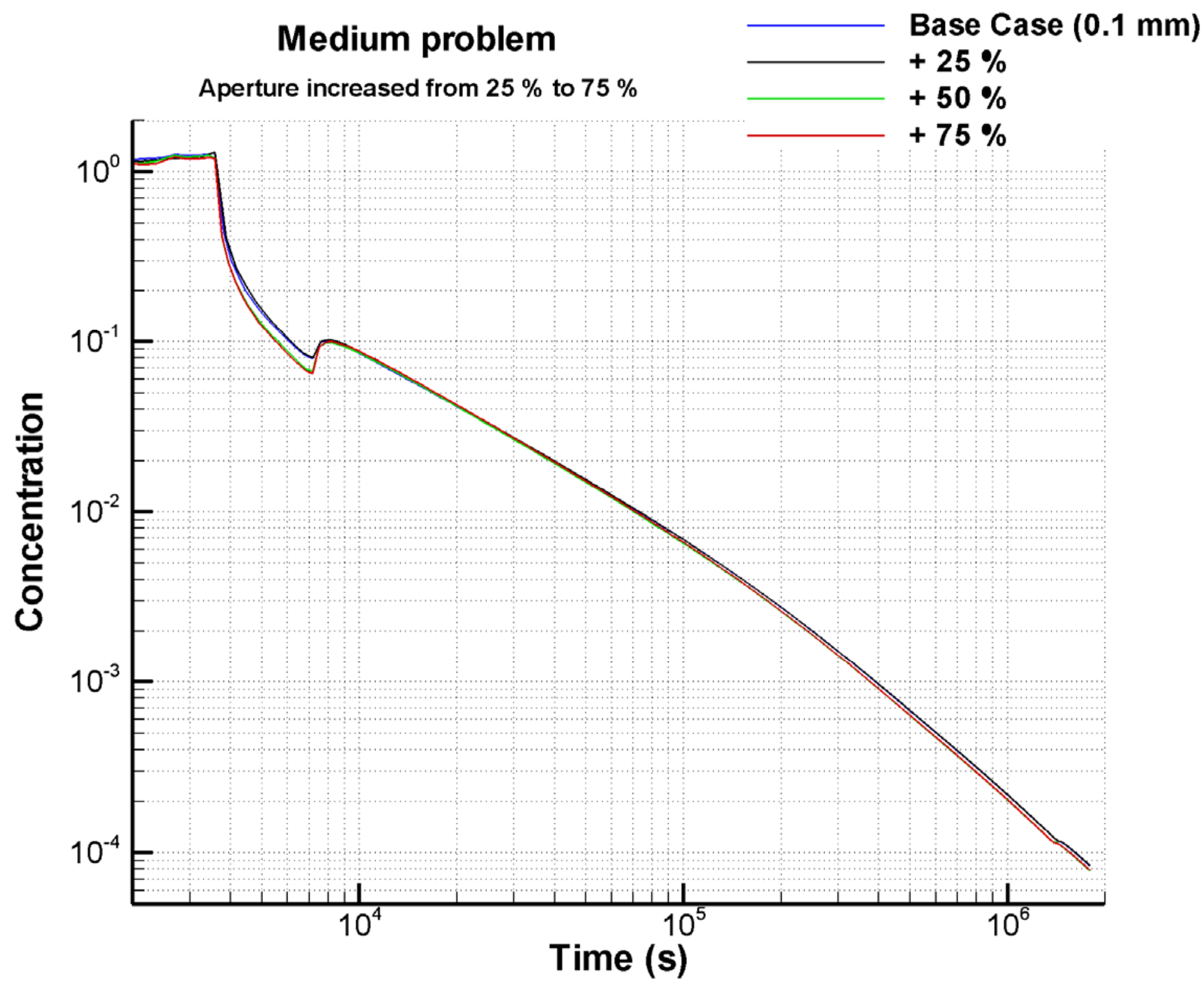


Figure $31: 1^{\text {st }}$ group of similar effects - BTC

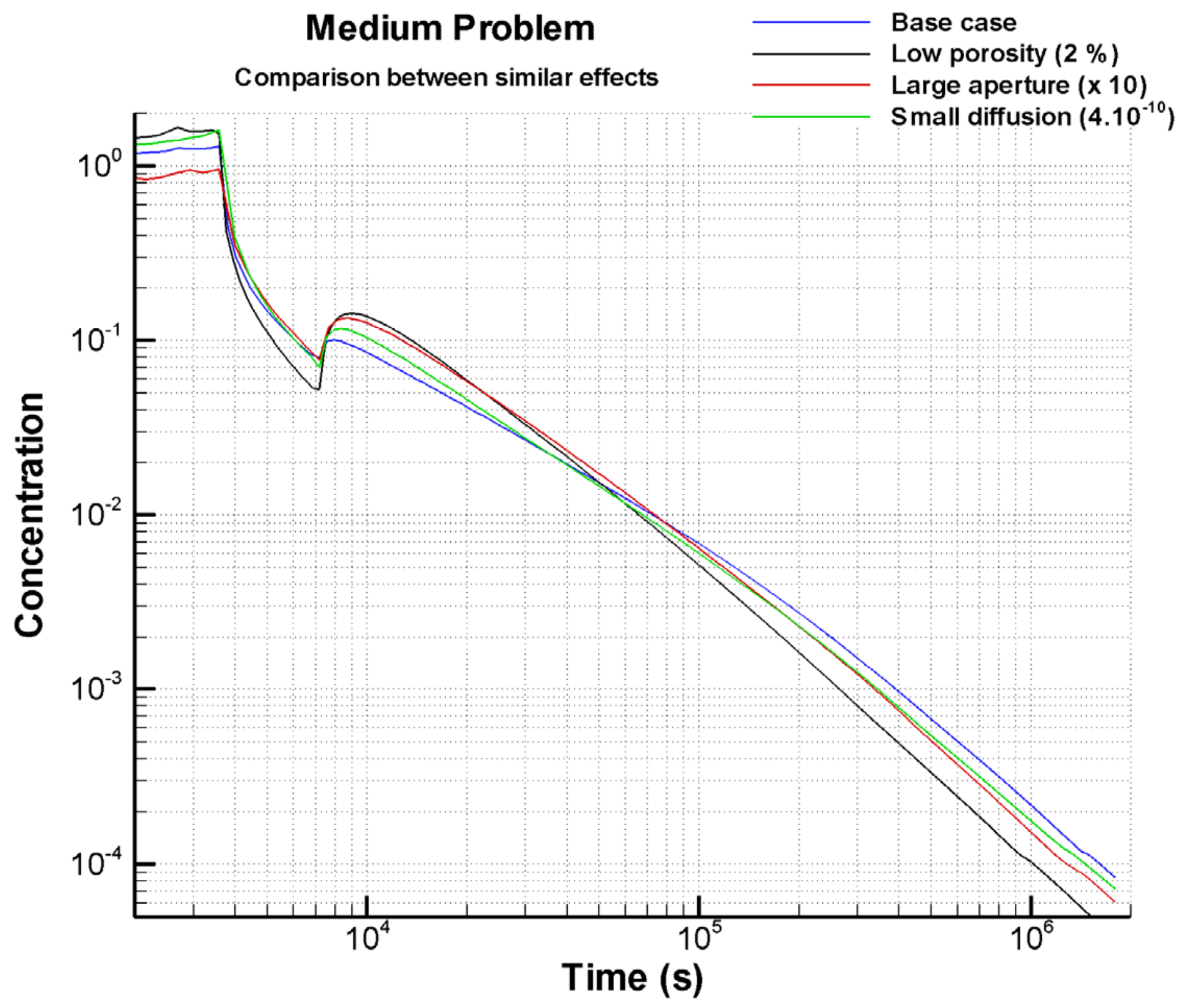


Figure 32: $1^{\text {st }}$ group of similar effects - Spatial Distribution

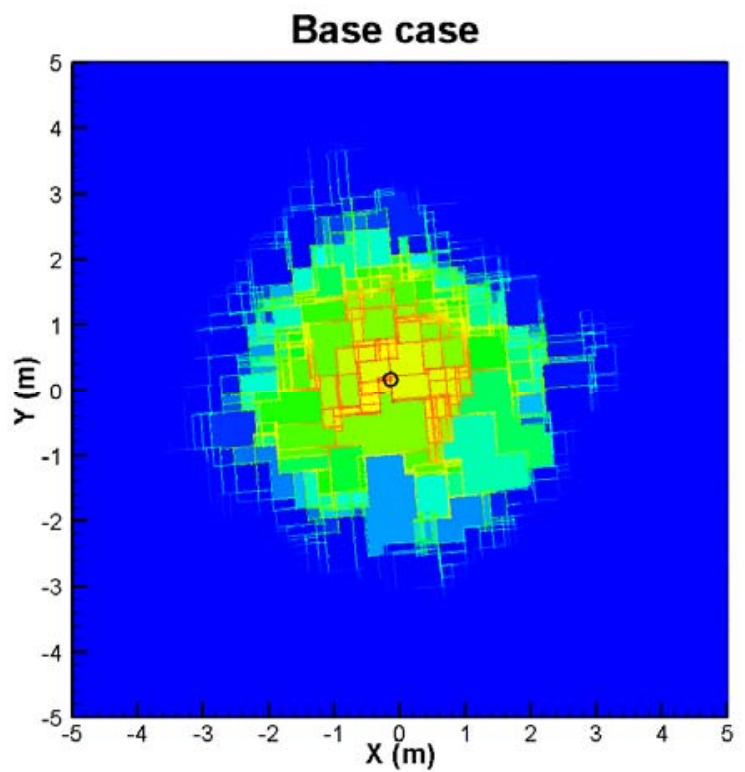

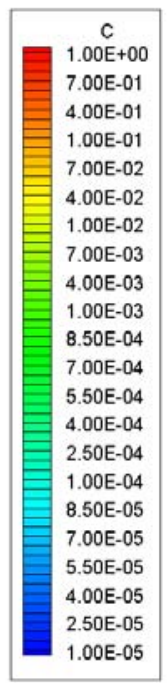

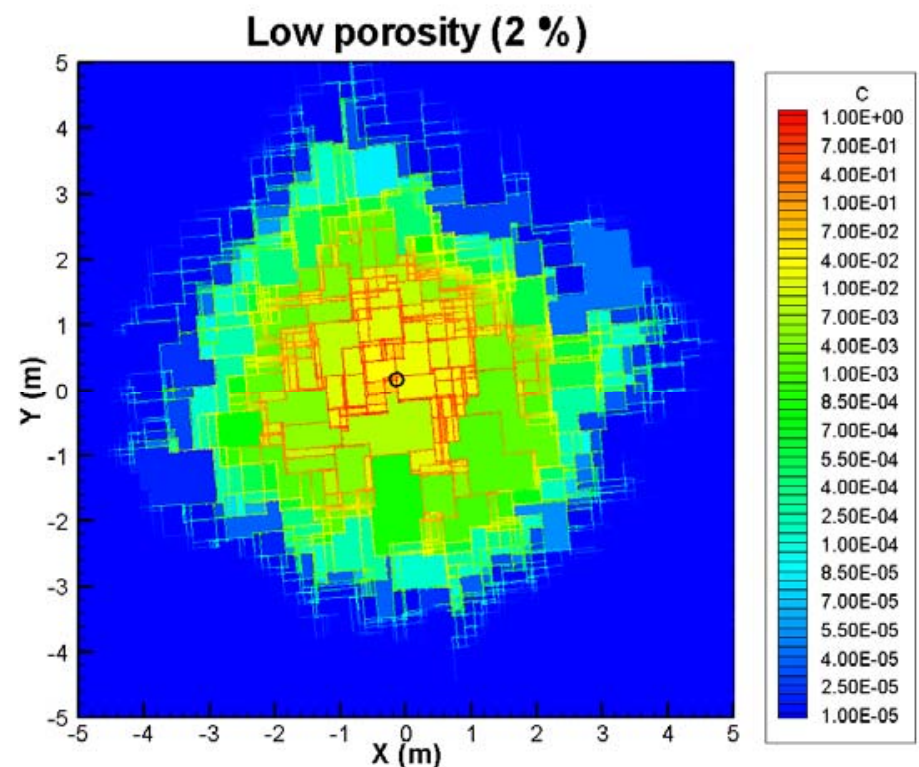

\section{Large aperture $(x 10)$}

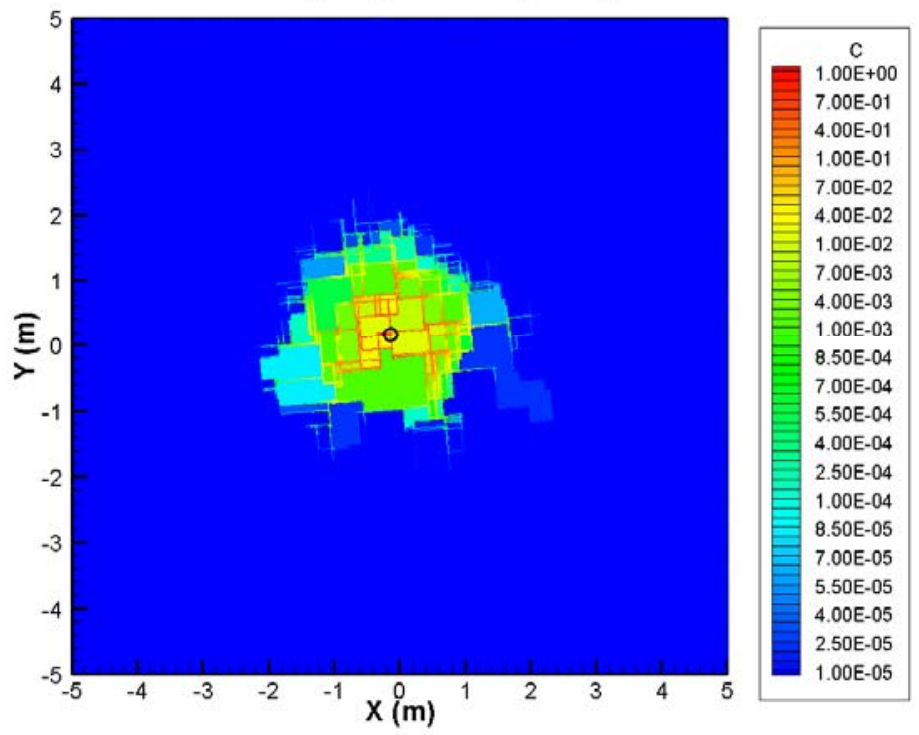

\section{End of injection Small diffusion $\left(4.10^{-10}\right)$}

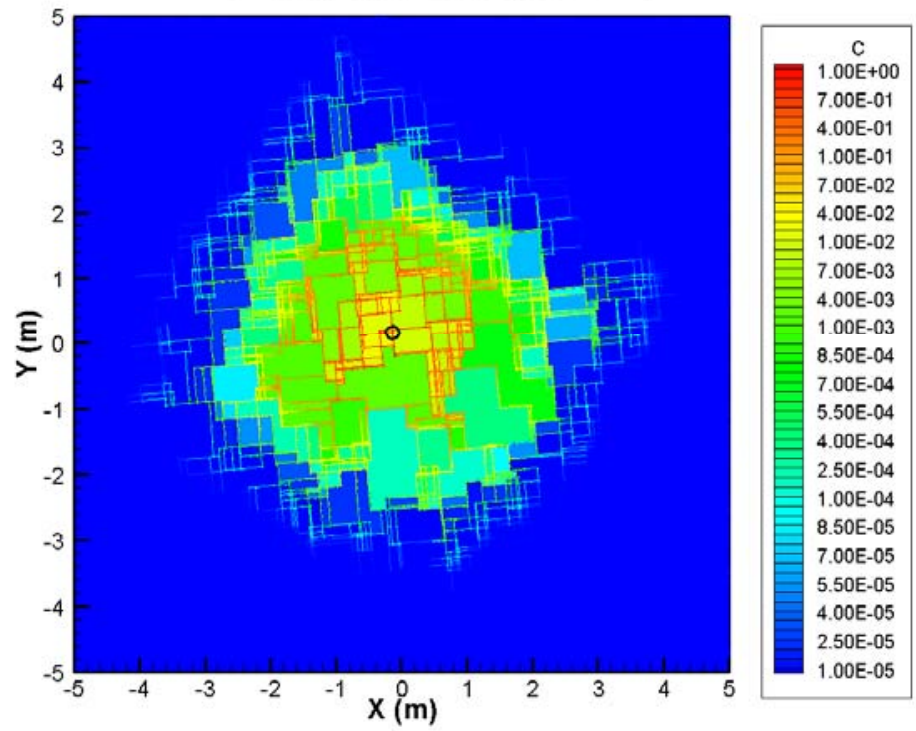


Figure $33: 2^{\text {nd }}$ group of similar effects - BTC

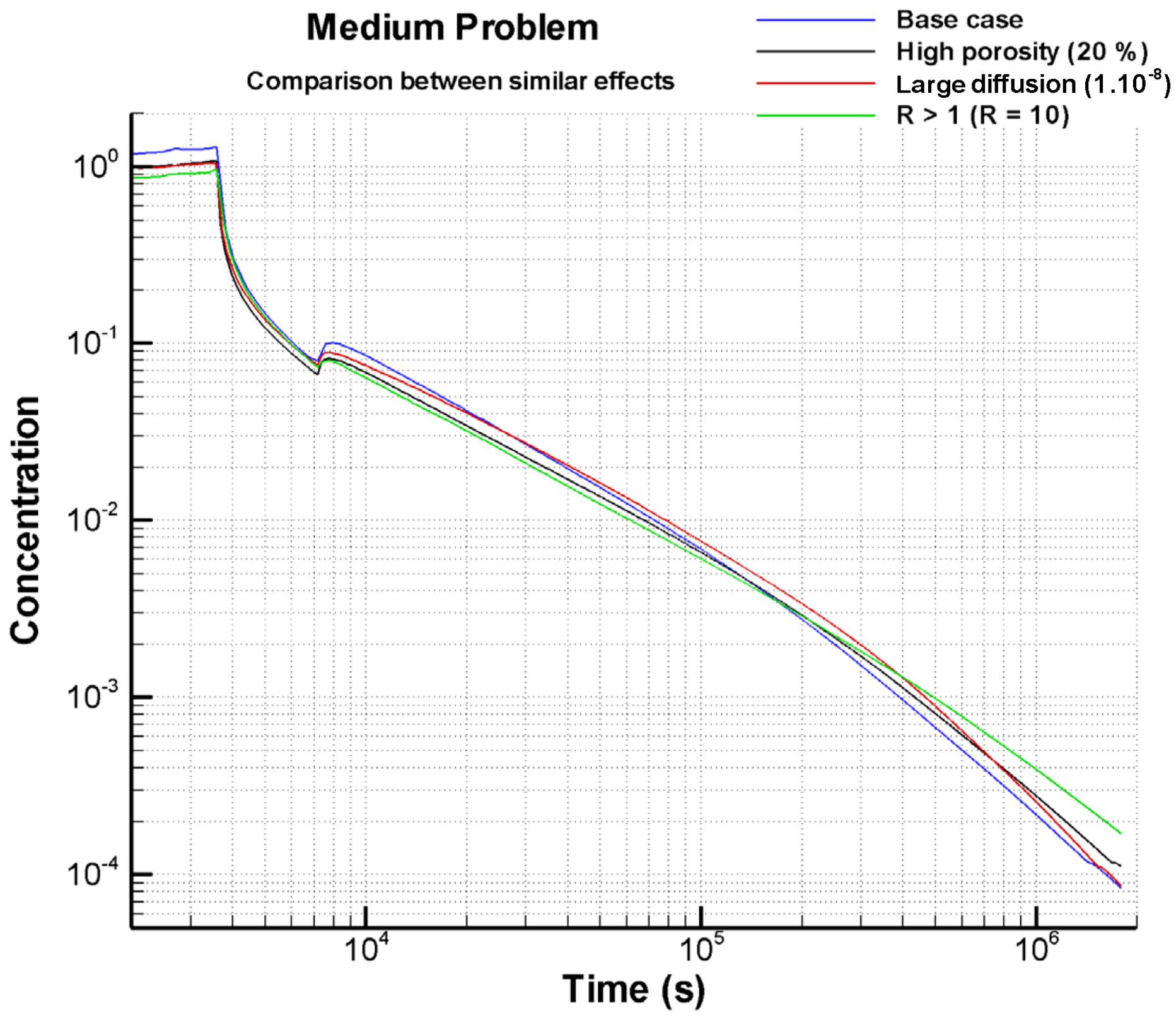


Figure 34: $2^{\text {nd }}$ group of similar effects - Spatial Distribution

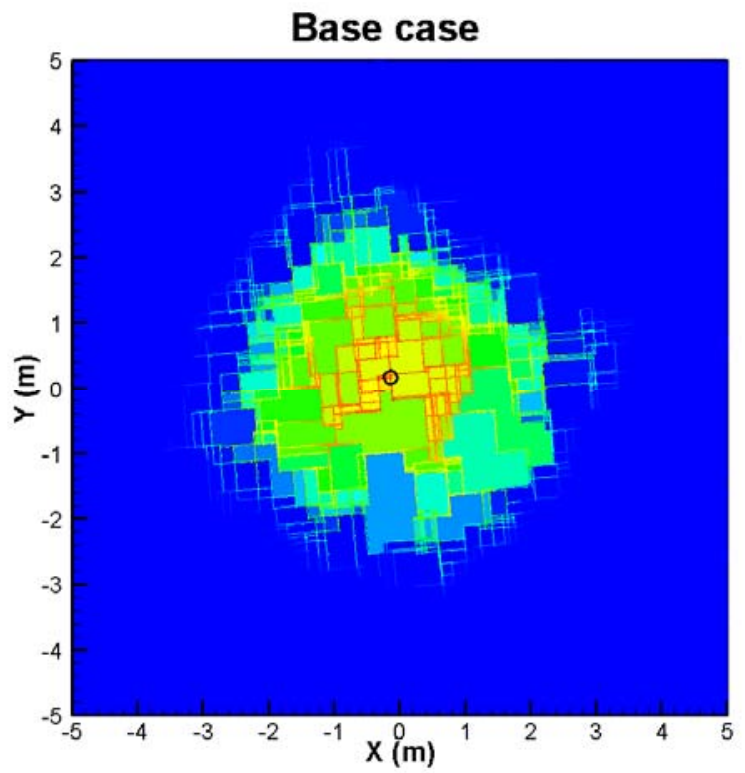

\begin{tabular}{|c|}
\hline$C$ \\
$1.00 \mathrm{E}+00$ \\
$7.00 \mathrm{E}-01$ \\
$4.00 \mathrm{E}-01$ \\
$1.00 \mathrm{E}-01$ \\
$7.00 \mathrm{E}-02$ \\
$4.00 \mathrm{E}-02$ \\
$1.00 \mathrm{E}-02$ \\
$7.00 \mathrm{E}-03$ \\
\hline $1.00 \mathrm{E}-03$ \\
$1.00 \mathrm{E}-03$ \\
$8.50 \mathrm{E}-04$ \\
$7.00 \mathrm{E}-04$ \\
$5.50 \mathrm{E}-04$ \\
$4.00 \mathrm{E}-04$ \\
$2.50 \mathrm{E}-04$ \\
$1.00 \mathrm{E}-04$ \\
$8.50 \mathrm{E}-05$ \\
$7.00 \mathrm{E}-05$ \\
$5.50 \mathrm{E}-05$ \\
$4.00 \mathrm{E}-05$ \\
$2.50 \mathrm{E}-05$ \\
$1.00 \mathrm{E}-05$ \\
\hline
\end{tabular}

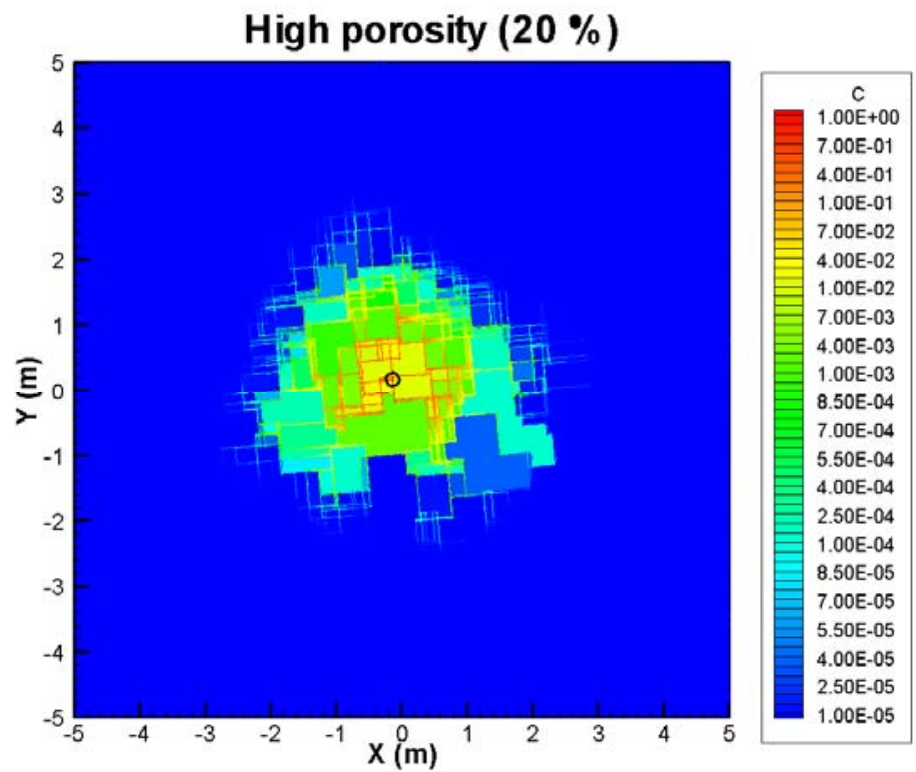

Large diffusion $\left(1.10^{-8}\right)$

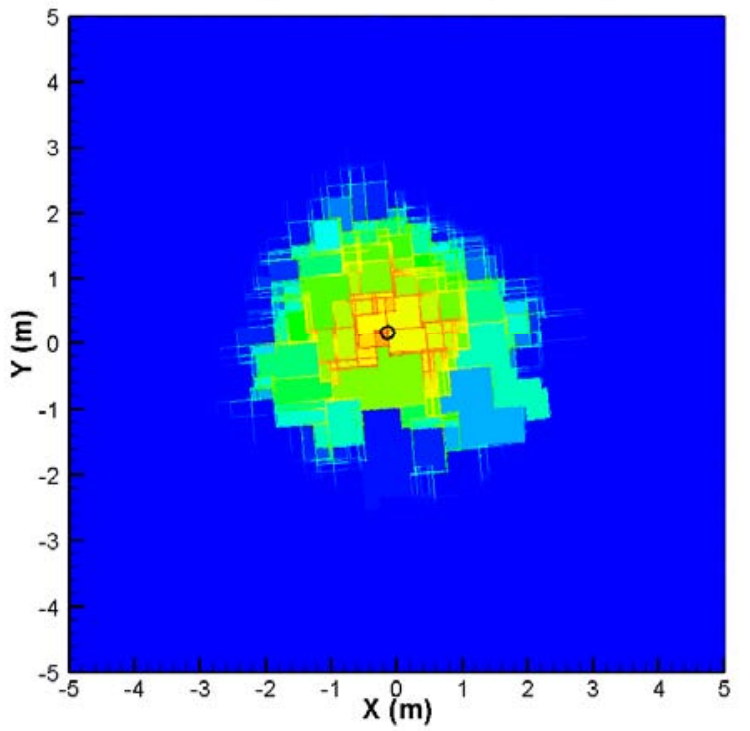

\section{End of injection}

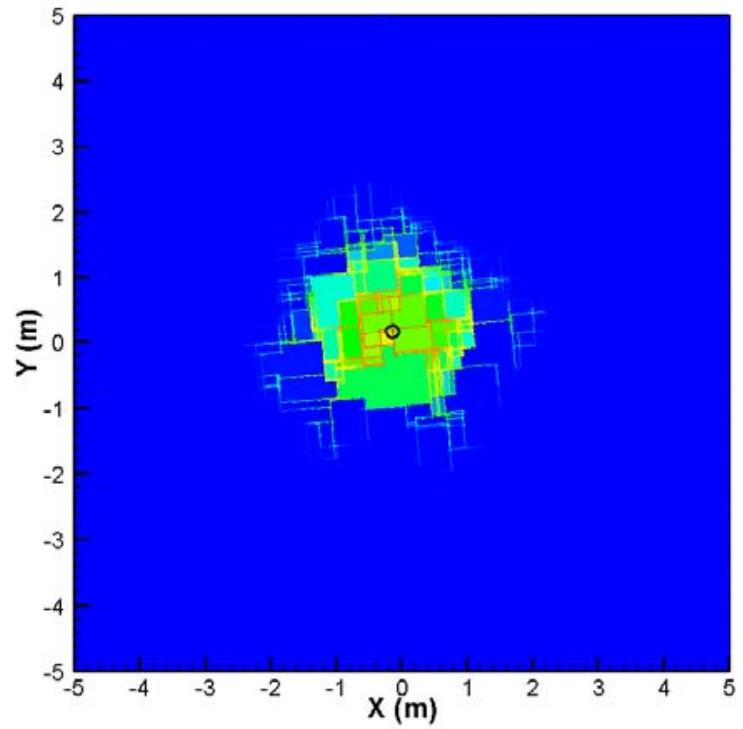

C $7.00 \mathrm{E}-01$ 4.00E-01 $1.00 \mathrm{E}-01$ 7.00E-02 $4.00 \mathrm{E}-02$ $1.00 \mathrm{E}-02$ $7.00 \mathrm{E}-03$ $4.00 \mathrm{E}-03$ $1.00 \mathrm{E}-03$ 8.50E-04

$7.00 \mathrm{E}-04$ $5.50 \mathrm{E}-04$ 4.00E-04 2.50E-04 $1.00 \mathrm{E}-04$ 8.50E-05 $7.00 \mathrm{E}-05$ $5.50 \mathrm{E}-05$ 4.00E-05 2.50E-05 $1.00 \mathrm{E}-05$ 
Figure $35: 3^{\text {rd }}$ group of similar effects - BTC

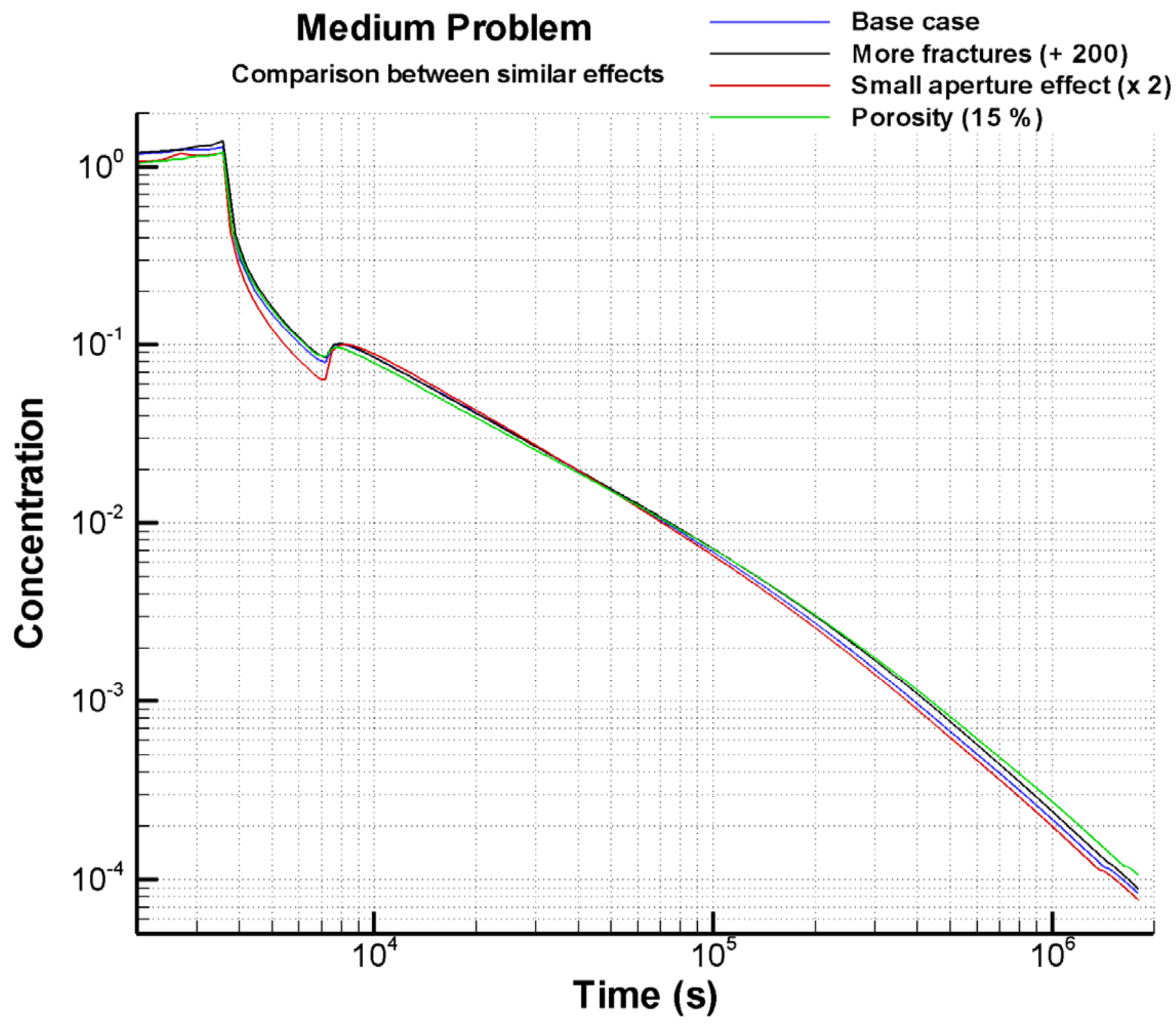


Figure 36: $3^{\text {rd }}$ group of similar effects - Spatial Distribution

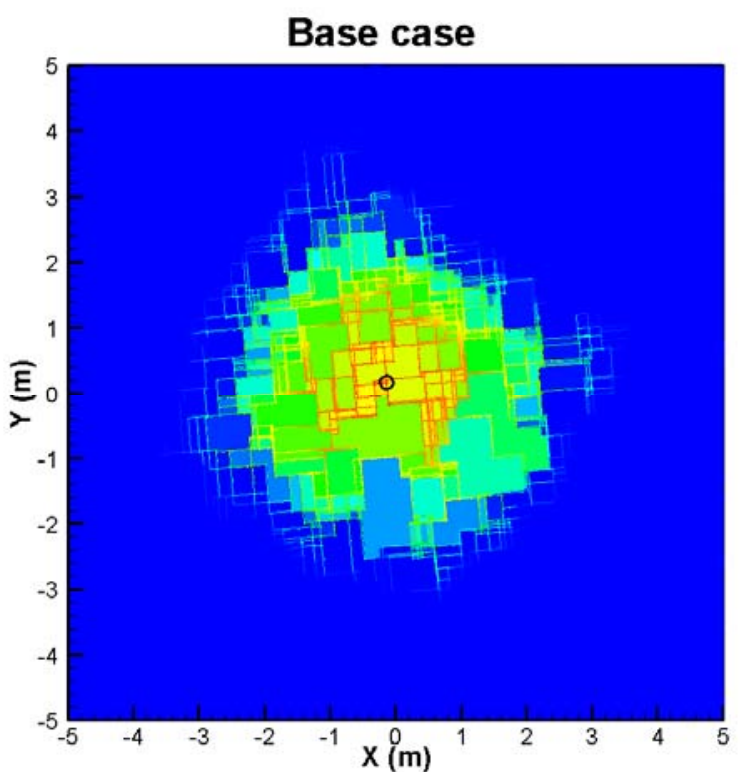

Small aperture effect $(x 2)$

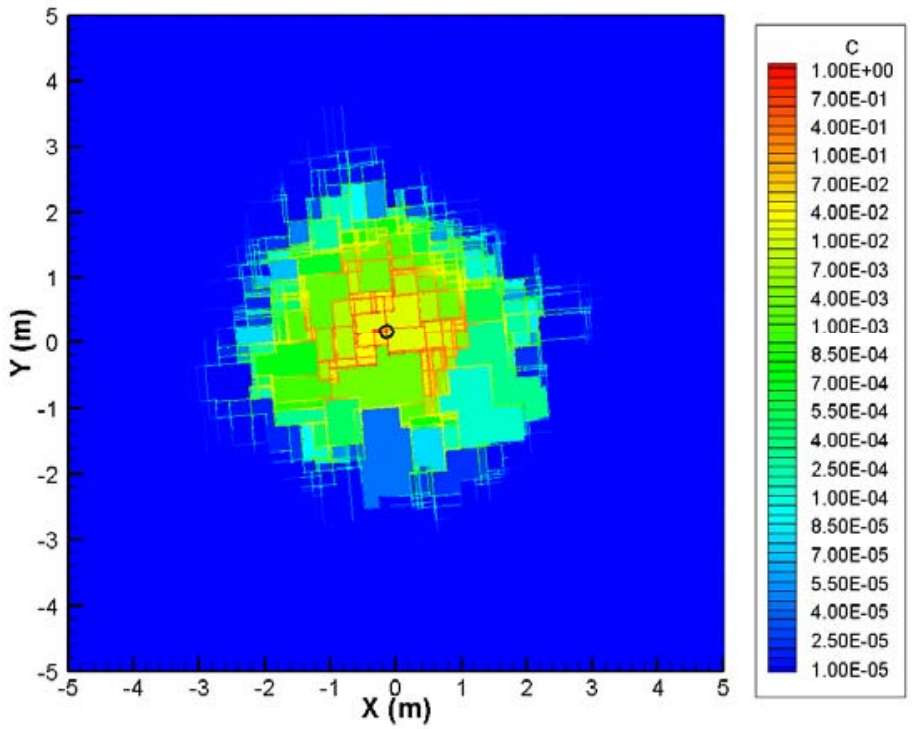

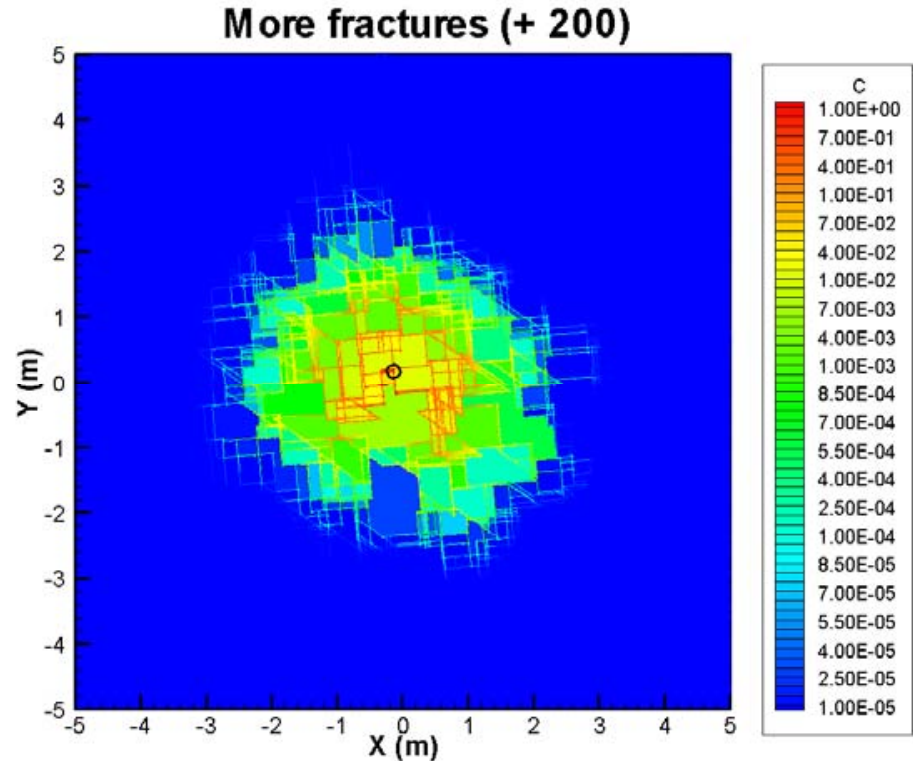

\section{End of injection}

\section{Porosity (15\%)}

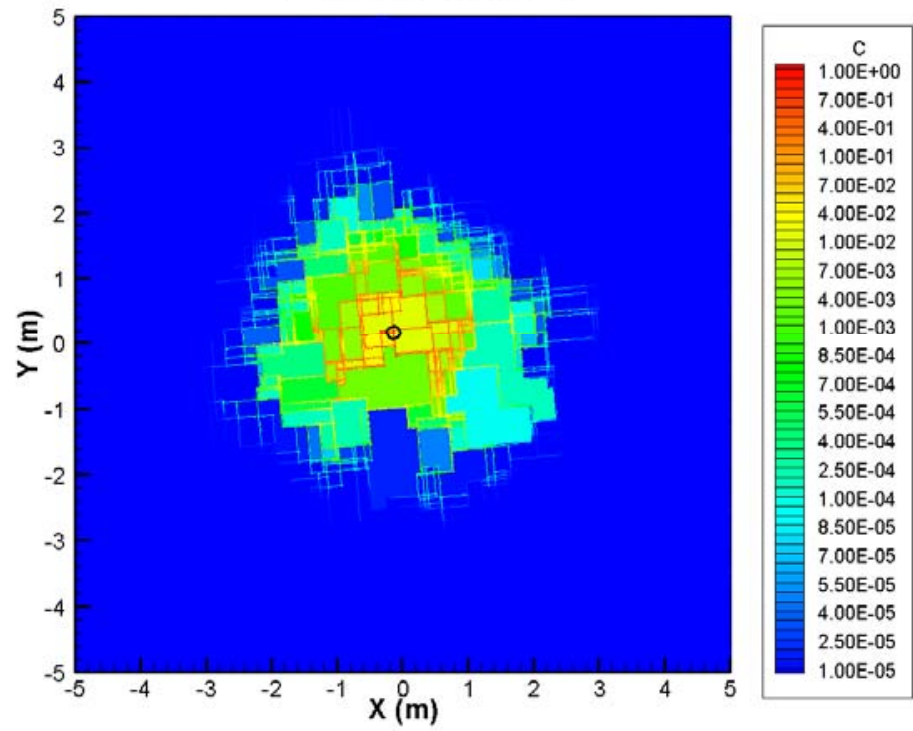




\section{DISCLAIMER}

This document was prepared as an account of work sponsored by the United States Government. While this document is believed to contain correct information, neither the United States Government nor any agency thereof, nor The Regents of the University of California, nor any of their employees, makes any warranty, express or implied, or assumes any legal responsibility for the accuracy, completeness, or usefulness of any information, apparatus, product, or process disclosed, or represents that its use would not infringe privately owned rights. Reference herein to any specific commercial product, process, or service by its trade name, trademark, manufacturer, or otherwise, does not necessarily constitute or imply its endorsement, recommendation, or favoring by the United States Government or any agency thereof, or The Regents of the University of California. The views and opinions of authors expressed herein do not necessarily state or reflect those of the United States Government or any agency thereof or The Regents of the University of California.

Ernest Orlando Lawrence Berkeley National Laboratory is an equal opportunity employer. 